\title{
12. TOWARD A HIGH-RESOLUTION STABLE ISOTOPE STRATIGRAPHY OF THE LAST 3.4 MILLION YEARS: SITES 658 AND 659 OFF NORTHWEST AFRICA ${ }^{1}$
}

\author{
M. Sarnthein ${ }^{2}$ and R. Tiedemann ${ }^{2}$
}

\begin{abstract}
Ocean Drilling Program Site 658, cored below a major upwelling cell offshore Cap Blanc, contains a largely undisturbed hemipelagic sediment section spanning the Brunhes Chron and the early Quaternary and late Pliocene. The companion Site 659 recovered a complete and undisturbed Neogene profile further offshore that serves as a nonupwelling pelagic reference section. Oxygen and carbon isotope ratios in benthic $(C$. wuellerstorfi and in part Uvigerina sp.) and planktonic foraminifers ( $G$. inflata) provide a climatic record of high resolution for the Brunhes Chron. At Site 658 the record extends back to the early Pleistocene and late Pliocene.

The standard oxygen isotope record of the last 730,000 $\mathrm{yr}$ is markedly refined by a well-documented high-frequency variation (e.g., by a new "aborted" ice age at stage 13.2 and by Younger-Dryas style climatic setbacks during most terminations). In the late Pliocene, the numerical oxygen isotope stage taxonomy was extended back to stage 137 about $3.3 \mathrm{Ma}$ ago. In comparison with published records, stage 114 at $2.7 \mathrm{Ma}$ represents the first major glaciation event, when ${ }^{18} \mathrm{O}$ was short-term enriched up to a middle Pleistocene glacial $\delta^{18} \mathrm{O}$ level. About $3.17 \mathrm{Ma}$ ago (stage 133), the interglacial oxygen isotope values of $C$. wuellerstorfi started to increase by $0.5 \%$ until $2.7 \mathrm{Ma}$ and then remained largely constant until the Holocene.

Based on the $\delta^{13} \mathrm{C}$ difference between $C$. wuellerstorfi and $G$. inflata, the dissolved $\mathrm{CO}_{2}$ in the ambient bottom water of Site 658 was dominated by the flux of particulate carbon from the overlying upwelling cell during the last $630,000 \mathrm{yr}$. In contrast, the advection of (upper) North Atlantic Bottom Water dominated in the control of the local $\mathrm{CO}_{2}$ content during the early Pleistocene and late Pliocene.
\end{abstract}

\section{INTRODUCTION}

Virtually undisturbed double and triple sediment sections obtained with the advanced hydraulic piston corer technique (APC) have greatly expanded the limits of precise chronostratigraphic resolution. Complete and highly detailed isotope stratigraphic records can now be established back to the early Quaternary and to the Cenozoic based on sediment cores from hemipelagic deep-sea sediment "piles" formed quasicontinuously at high accumulation rates. In this chapter, we summarize a new set of stable isotope data with fine-scale resolution generated by a close examination of Ocean Drilling Program (ODP) Site 658 and the upper sections of Site 659. These data form the base for a number of paleoceanographic and paleoclimatic studies published in this volume that rely on precise accumulation and flux rate data, for example, of siliciclastic, opaline, and pollen sediments (Dupont et al., this vol.; Stabell, this vol.; Tiedemann et al., this vol.).

A major target of Leg 108 was to study the history of oceanic upwelling and continental sediment supply offshore from the west Sahara (Fig. 1). Below the coastal upwelling cell offshore from Cap Blanc, Site 658 recovered a largely undisturbed, thick hemipelagic sediment section at $2271 \mathrm{~m}$ water depth. Shipboard stratigraphic data revealed average sedimentation rates of about $15 \mathrm{~cm} / 1000 \mathrm{yr}$ in the upper $100 \mathrm{mbsf}$. Further below, a hiatus lasting for about $840,000 \mathrm{yr}$ is preceded by sedimentation rates of almost $8 \mathrm{~cm} / 1000 \mathrm{yr}$ down to $163 \mathrm{mbsf}$ (i.e., about $2.4 \mathrm{Ma}$ ), and of about $11 \mathrm{~cm} / 1000 \mathrm{yr}$ down to 300 mbsf (slightly modified from Ruddiman, Sarnthein, et al., 1988, pp. 105-219, cf. Table 5). These high rates of sediment accumulation provided a Pliocene-Pleistocene

\footnotetext{
${ }^{1}$ Ruddiman, W., Sarnthein, M., et al., 1989. Proc. ODP, Sci. Results, 108: College Station, TX (Ocean Drilling Program).

2 Geologisch-Paläontologisches Institut, Universităt Kiel, Olshausenstrasse 40, D-2300 Kiel, Federal Republic of Germany.
}

sediment record of high quality in which bioturbation hardly obscures the fine-scale resolution of short-term features in climate stratigraphy.

In comparison, at nonupwelling neighbor Site 659 , which lies more distant from the continent on top of the Cape Verde Ridge in $3081 \mathrm{~m}$ water depth (Fig. 1), we cored a section that consists largely of pelagic carbonate sediments. Sedimentation rates continuously averaged $3 \mathrm{~cm} / 1000 \mathrm{yr}$ down to 140 mbsf, and carbonate dissolution was low (Ruddiman, Sarnthein, et al., 1988, pp. 221-325).

Both the carbon and the planktonic foraminifer oxygen isotopic records at Site 658 are expected to reflect the interplay of such global and local oceanographic processes as the upwelling of cold subsurface water and the excessive production of isotopically light organic carbon. Alternatively, the records of Site 659 are expected to represent the "general" isotopic history of the ocean in subtropical latitudes.

\section{METHODS}

\section{Stable Isotope Analysis}

At Site 658 , stable oxygen and carbon isotope analyses were made at (irregular) sample intervals averaging $36 \mathrm{~cm}$ between 0 and $100 \mathrm{mbsf}$ and $70 \mathrm{~cm}$ from 100 to $300 \mathrm{mbsf}$. From 0 to $100 \mathrm{mbsf}$, this spacing corresponds to an average interval of about $2400 \mathrm{yr}$ and from 100 to $273 \mathrm{mbsf}$, to an interval of $6600 \mathrm{yr}$. Six major data gaps in the combined $\delta^{18} \mathrm{O}$ records of $C$. wuellerstorfi and Uvigerina sp. at Site 658 make up 15 k.y. at 160.5-161.8 mbsf composite depth (c.d.), 20 k.y. at 172.5-174.5 mbsf (c.d.), 26 k.y. at 181.4-183.9 mbsf (c.d.), 36 k.y. at 190.25-193.75 mbsf (c.d.), 21 k.y. at 219.5-222.0 mbsf (c.d.), and 30 k.y. at 266.06-269.74 mbsf (c.d.).

Further below, gaps at $272.87-279.25$ and $279.72-288.77 \mathrm{mbsf}$ (c.d.) are about 52 and 75 k.y., respectively. Moreover, a precise comparison of our $\delta^{18} \mathrm{O}$ record with that of Raymo et al. (1988) revealed a coring gap spanning up to $9.4 \mathrm{~m}$ at $154.94-155.05 \mathrm{mbsf}$ (c.d.) in Core 108-658A-18H, where drilling changed from APC to XCB coring (see below). Based on a removal of these gaps, the actual average sampling resolution at $100-153 \mathrm{mbsf}$ amounts to one sample 


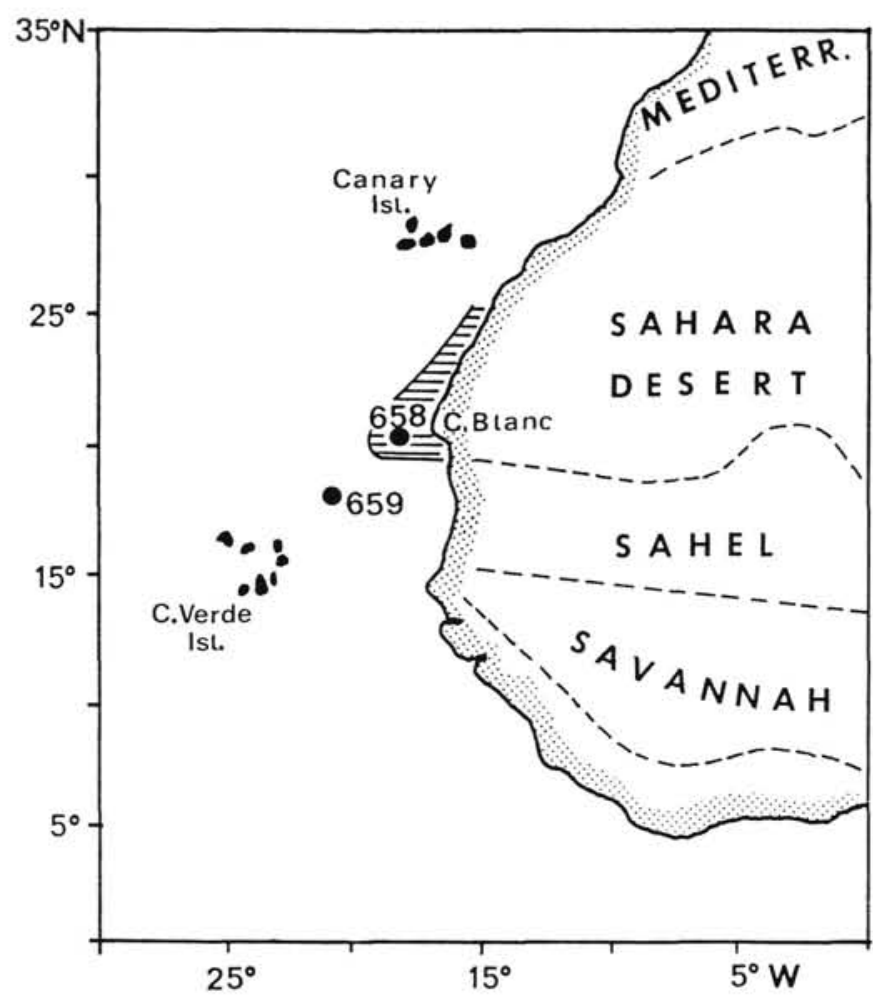

Figure 1. Location map for Sites $658\left(20^{\circ} 44.95^{\prime} \mathrm{N}, 18^{\circ} 34.85^{\prime} \mathrm{W}\right)$ and 659 $\left(18^{\circ} 04.63^{\prime} \mathrm{N}, 21^{\circ} 01.57^{\prime} \mathrm{W}\right)$

per $58 \mathrm{~cm}$ or every $6300 \mathrm{yr}$ in the remaining proportions of the sediment profile. At $155-273$ mbsf (c.d.), it increases to one sample per $56 \mathrm{~cm}$ or every $5240 \mathrm{yr}$ (based on the sedimentation rates of Table 5).

At Site 659, 0-33 mbsf, the average sampling interval was $29 \mathrm{~cm}$, equivalent to $8800 \mathrm{yr}$ according to Leg 108 shipboard stratigraphy (Ruddiman, Sarnthein, et al., 1988).

Four foraminifer species were used for stable isotope analyses: Globorotalia inflata (about 20 specimens per sample, $250-315 \mu \mathrm{m}$ size fraction) recorded the history of the subsurface ocean, particularly the regime near and below the thermocline (Deuser et al., 1981; Ganssen and Sarnthein, 1983). On the other hand, Cibicidoides wuellerstorfi (1-10 specimens per sample from the $315-400-\mu \mathrm{m}$ size fraction) provided a parallel record of the history of Intermediate North Atlantic deep water. Furthermore, both Uvigerina peregrina and $U$. auberiana (1-15 specimens, $315-400 \mu \mathrm{m}$, per sample) were analyzed at Site 658 at 150-300 mbsf. They provide a second benthic oxygen isotope record where the record of $C$. wuellerstorfi is incomplete because specimens were absent in the sediment. Eighty-two twin measurements demonstrate the oxygen isotopic match between $U$. peregrina and $U$. auberiana (Fig. 2).

Standard techniques for analysis were used: reaction with $100 \%$ orthophosphoric acid at $70^{\circ} \mathrm{C}$ and removal of water with the CARBOKIEL automated preparation system, and isotopic analysis in a Finnigan MAT 251 mass spectrometer at the C-14 Laboratory of Kiel University. Both instruments were calibrated to the PEE DEE Belemnite (PDB) scale through National Bureau of Standards NBS-19 and NBS-20 carbonate standards. External reproduction capabilities (including $\mathrm{CO}_{2}$ preparation and spectrometric analysis) were $\pm 0.07 \%$ o $\delta^{18} \mathrm{O}$ and $\pm 0.04 \% 0 \quad \delta^{13} \mathrm{C}$ (both 1 -sigma values). A more complete description of these techniques is given in Ganssen (1983) and Zahn (1986).

\section{Revision of Composite Depth Sections}

In order to obtain complete isotopic records, two holes were sampled at Site 658 and three at Site 659. In general, our depth correlation between the different holes at each site is based on the composite depth sections reported by the Leg 108 shipboard party

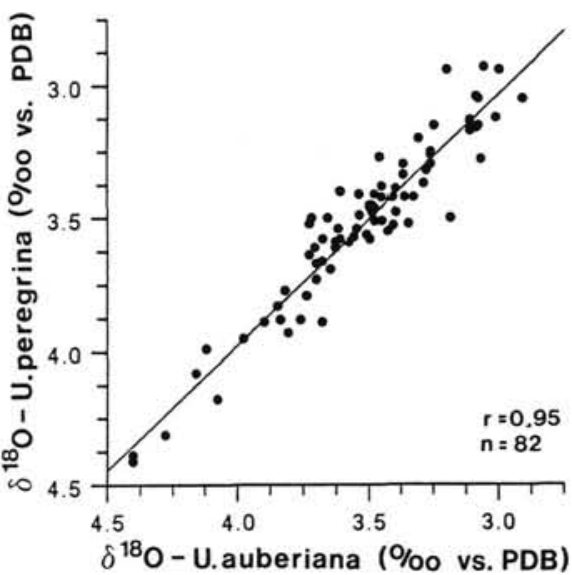

Figure 2. Correlation between $\delta^{18} \mathrm{O}$ values of Uvigerina auberiana and $U$. peregrina from Site 658, 151-293 mbsf (c.d.).

(Ruddiman, Sarnthein, et al., 1988). However, careful inspection of the fluctuations in our oxygen isotopic curves suggested a revision of the correlation between Holes 658A and 658B for the uppermost three cores (see Fig. 3B and new "pathway" in Table 1).

The new between-hole correlation pattern is in harmony with and supplemented by a revised interpretation of the best fit between the continuous magnetic susceptibility curves of both holes based on some marked short-term fluctuations (Fig. 3A). This revision results in a general reduction of composite depth values by $2.85 \mathrm{~m}$ in all cores of Hole 658A below $15 \mathrm{mbsf}$ (c.d.) compared with the values defined by Ruddiman, Sarnthein, et al. (1988), who used Hole 658A as major reference section.

The composite depth section of Site 658 leads to a largely complete overlap of sections of "good core" between Holes 658A and 658B down to a composite depth of $\sim 85$ mbsf (discussion of gaps see below). Moreover, in this composite section we increased markedly the quality of the sediment section at Site 658 by closing "on paper" numerous gaps artificially induced by gas in the otherwise uncontorted core sections, based on a detailed computerized data record of all voids provided by W. B. Curry (pers. comm.).

Based on the revised data set of Bloemendal et al. (1988), the composite depth section of the three holes at Site 659 also had to be readjusted in the following manner. From $0-3.73 \mathrm{mbsf}$, the calculation of the composite depth is based on Hole 659B because the top 33 - and $21-\mathrm{cm}$ sediment sections were lost from Holes $659 \mathrm{~A}$ and $659 \mathrm{C}$, respectively. From 8.9 to 19.0 mbsf c.d., the section from Hole $659 \mathrm{C}$ replaces the one from Hole $659 \mathrm{~A}$ as the major reference unit (see new "pathway" in Table 2); it appears more likely that Core $108-659 \mathrm{~A}-2 \mathrm{H}$ lost $1.1-1.5 \mathrm{~m}$ of sediment than the wellpreserved Core 108-659C-2H expanded by the same amount. Further below, the composite depth values remain as defined by Ruddiman, Sarnthein, et al. (1988), except for a minor revision at 101-112.5 mbsf (Table 2).

\section{RESULTS}

All data analyzed are given in Tables 3 and 4 . Figure 4 depicts the planktonic and benthic oxygen and carbon isotope records of Site 658, a 294-m-long sediment section reaching back to $3.6 \mathrm{~m}$.y. Figure 5 depicts the equivalent information for the topmost $31 \mathrm{~m}$ of Site 659 equating the past $1.0 \mathrm{~m} . \mathrm{y}$.

The oxygen isotopic stages of the last $0.73 \mathrm{Ma}$ were identified by visual graphic correlation using features defined by Pisias et al. (1984) and Prell et al. (1986) and dated by Imbrie et al. (1984) and Martinson et al. (1987). The earlier oxygen isotopic fluctuations beginning at the top of the Olduvai Subchron (Site 658: $106.65 \mathrm{msbf}$, c.d.) were identified as midpoint stages $60-63$ in line with the classification scheme of Ruddiman et al. (1986) and as stages 64-116 in line with the scheme of Raymo et al. (1988). 
A

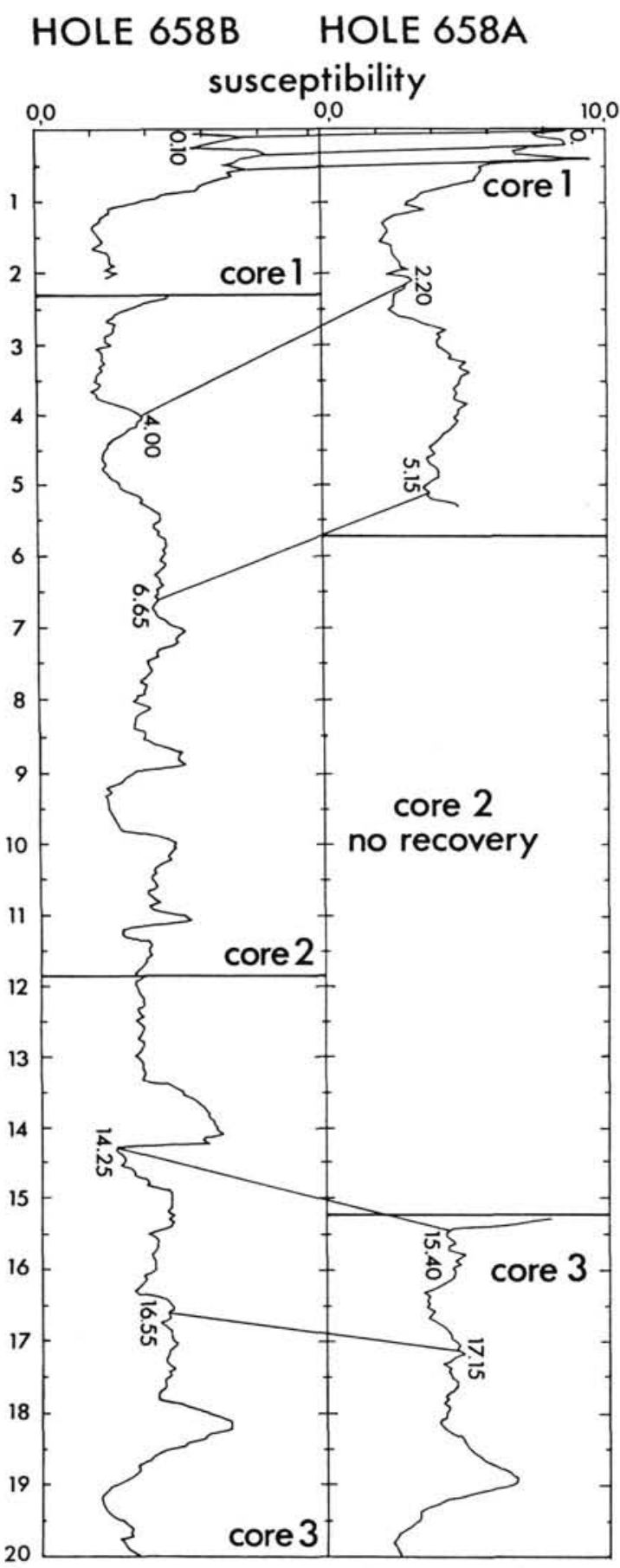

B

HOLE 658B
HOLE 658A

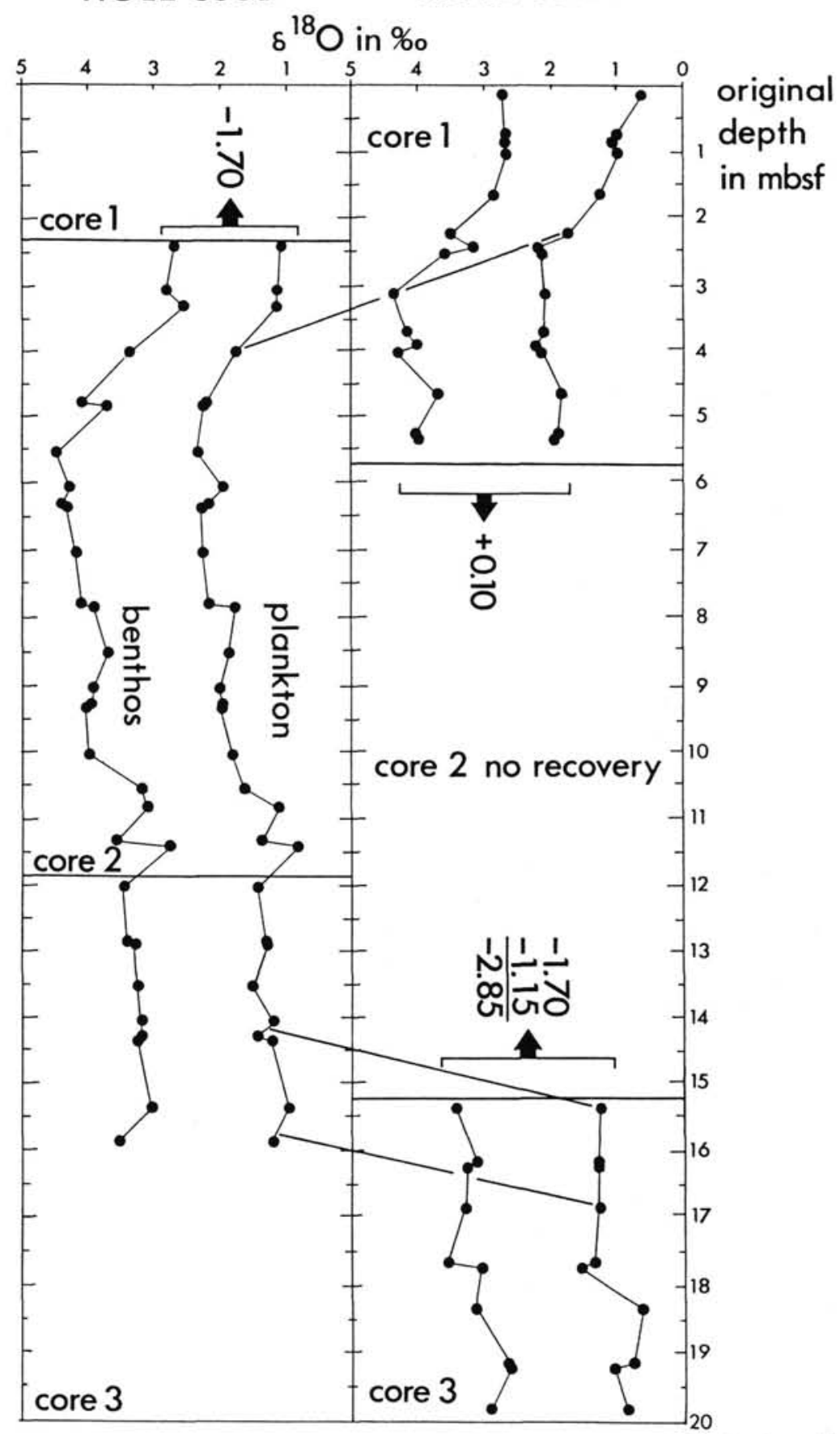

Figure 3. New depth correlations between Holes 658A and 658B to 20 mbsf (nominal depth of penetration). For the between-hole "pathway" of the composite depth, compare with Table 1 . Original depth $=$ uncorrected depth values obtained by shipboard party. The $\delta^{18} \mathrm{O}$ events 3.33 and 4 are lost because of a stratigraphic gap in Section 108-658B-2H-6. A. Magnetic susceptibility records (from Bloemendal et al., 1988). B. Oxygen isotope records.

Further downcore, the high stratigraphic resolution of the benthic oxygen isotope record at Site 658 (1 sample/5240 yr) enabled us to propose a new nomenclature for the preceding sequence of oxygen isotope stages 116-137, which make up the time span back to more than 3.3 Ma. Most isotopic events are well defined by several data points along the stage transitions. At 3.41 Ma C. wuellerstorfi largely ceases to occur in our samples and also the $\delta^{18} \mathrm{O}$ record of Uvigerina sp. is incomplete due to inadequate core recovery.

Pliocene age control points and the approximate midpoint ages assigned to stages $61-137$ are summarized in Table 5 . Ages were derived by linear interpolation between the top Olduvai and Réunion I magnetic events, which were refined by the tuned TP607 time scale of Raymo et al. (1988) and the 
Table 1. New "pathway" of composite depth levels to correlate core sections in Holes $658 \mathrm{~A}$ and $658 \mathrm{~B}$ to $20 \mathrm{mbsf}$.

\begin{tabular}{ccc}
\hline $\begin{array}{c}\text { Hole 658B } \\
\text { Original depth } \\
\text { (mbsf) }\end{array}$ & $\begin{array}{c}\text { Hole 658A } \\
\text { Original depth } \\
\text { (mbsf) }\end{array}$ & $\begin{array}{c}\text { Site 658 } \\
\text { Composite depth } \\
\text { (mbsf) }\end{array}$ \\
\hline 0.10 & 0 & 0.10 \\
4.00 & 2.20 & 2.30 \\
6.65 & 5.15 & 4.95 \\
6.81 & 5.31 & 5.11 \\
14.25 & 15.40 & 12.55 \\
$(16.55)$ & $(17.15)$ & $(14.30)$ \\
\hline
\end{tabular}

Gauss-Gilbert magnetic boundary. Furthermore, we used paleomagnetically dated and quantitatively determined biostratigraphic datums, especially below 150 mbsf (Ruddiman, Sarnthein, et al., 1988; Chepstow-Lusty et al., this vol.; in part, ages revised by Raymo et al., 1988).

A close comparison of our oxygen isotope record with that of Site 607 (Raymo et al., 1988) led to a particularly clear morphological identification of stages $75,78,82,88,96$, and 100. Moreover, the comparison demonstrated that we missed several cycles (stages 89-95) in our record at about 155 mbsf (c.d.), a depth that precisely matches the loss of Core 108$658 \mathrm{~A}-18 \mathrm{H}$. Accordingly, we regard this gap as an artifact.

Both the oxygen and carbon isotope curves of Site 658 provide detailed records of climate history with many novel features from the last $730,000 \mathrm{yr}$ and new information from 1.57 to $3.4 \mathrm{Ma}$. However, the records are interrupted by a major erosional hiatus ending near the Brunhes-Matuyama boundary and two small ones at $\delta^{18} \mathrm{O}$ stages 4 and 18 , which all require careful identification (see discussion chapter).

The hemipelagic sedimentation rates at Site 658 vary from 9 to $26 \mathrm{~cm} / \mathrm{k}$.y. in the uppermost $100 \mathrm{~m}$ and around $7-12$ $\mathrm{cm} / \mathrm{k}$.y. below $100 \mathrm{mbsf}$ (Table 5), thus exceeding normal pelagic sedimentation rates by a factor of $6-8$. Hence, we assume that the full range of glacial-to-interglacial oxygen isotope variations, which is commonly obscured by bioturbation in lower deposition-rate records, remained almost intact at Site 658 during the Brunhes Chron. The isotopic range in the benthic record reached a maximum of $2.35 \%$ at the stage 11-12 boundary. In the values of the subsurface planktonic species $G$. inflata, which is likely to show a smoothed record of extreme sea-surface temperature (SST) fluctuations because of its habitat at about $100-400 \mathrm{~m}$ water depth, the variations in $\delta^{18} \mathrm{O}$ only reached $2.2 \%$ (at the stage $9-10$ boundary).

As expected from the differences in sedimentation rates, the maximum range of benthic $\delta^{18} \mathrm{O}$ variation at Site 658 slightly exceeds that of neighboring Site 659 by up to $0.2 \%$ and exactly matches that of the high-resolution core M 12392 (Zahn et al., 1986). The range difference between Sites 658 and 659 is considerably greater (by $0.5 \%$ ) for planktonic $\delta^{18} \mathrm{O}$ variations, which may result from locally enhanced SST fluctuations along with the coastal upwelling cell at Site 658. A future comparison of these data with the ketone-based temperature record presented by Poynter et al. (this vol.) might lead to a better understanding of this question.

At Site 659, the benthic and planktonic oxygen isotope records (Fig. 5) generally match closely. They are of standard quality, with surprisingly uniform intermediate sedimentation rates (Table 6). Minor irregularities in assigning stage names (22-27) occur at 25-30 mbsf, probably a result of too wide a sampling interval. Furthermore, the magnitude of peaks at events 19.1 and 18.3 appears atypical, especially in the plank-

Table 2. New "pathway" of composite depth levels to correlate core sections in Holes 659A, 659B, and 659C to 119 mbsf.

\begin{tabular}{|c|c|c|c|}
\hline $\begin{array}{l}\text { Hole } 659 \mathrm{~A} \\
\text { Original depth } \\
\text { (mbsf) }\end{array}$ & $\begin{array}{l}\text { Hole 659B } \\
\text { Original depth } \\
\text { (mbsf) }\end{array}$ & $\begin{array}{l}\text { Hole 659C } \\
\text { Original depth } \\
\text { (mbsf) }\end{array}$ & $\begin{array}{c}\text { Site } 659 \\
\text { Composite depth } \\
\text { (mbsf) }\end{array}$ \\
\hline & $0.21 \bullet$ & 0.04 & ${ }^{\mathrm{a}} 0.21$ \\
\hline 0.06 & 0.39 & 0.25 & ${ }^{\mathrm{a}} 0.39$ \\
\hline 0.25 & 0.58 & 0.46 & ${ }^{\mathrm{a}} 0.58$ \\
\hline 0.96 & $1.21 \downarrow$ & 1.12 & ${ }^{\mathrm{a}} 1.21$ \\
\hline 1.77 & $2.05^{\circ}$ & 2.02 & $a_{2.05}$ \\
\hline 2.53 & 2.71 & 2.71 & ${ }^{\mathrm{a}} 2.71$ \\
\hline 2.86 & $2.98 \downarrow$ & 2.98 & $a_{2.98}$ \\
\hline \multirow[t]{2}{*}{3.40} & 3.43 & 3.43 & ${ }^{a} 3.43$ \\
\hline & $\underline{3.73} \bullet \rightarrow-$ & $\rightarrow \cdot \underline{3.73}$ & $a_{3.73}$ \\
\hline 3.73 & $\overline{3.82}$ & $\overline{3.85}$ & ${ }^{a} 3.85$ \\
\hline 4.09 & 4.15 & 4.21 & $a_{4.21}$ \\
\hline 4.54 & 4.57 & 4.63 & ${ }^{\mathrm{a}} 4.63$ \\
\hline 5.08 & & $\downarrow 5.11$ & ${ }^{a_{5} .11}$ \\
\hline 5.35 & 5.29 & 5.38 & ${ }^{a_{5} .38}$ \\
\hline 5.50 & 5.44 & 5.50 & ${ }^{\mathrm{a}} 5.50$ \\
\hline 5.83 & 5.74 & 5.83 & $a_{5.83}$ \\
\hline 6.19 & 6.07 & \multirow[t]{2}{*}{$\downarrow$} & a $(6.19)$ \\
\hline $6.50($ core 1$)$ & 6.50 & & $b_{(6.50)}$ \\
\hline $7.80($ core 2$)$ & & $8.90($ core 1$)$ & ${ }^{b} 8.90$ \\
\hline 11.00 & & $\downarrow 12.10$ (core 2$)$ & $b_{12.10}$ \\
\hline 14.30 (core 2 ) & & 15.80 & $\mathrm{a}_{15.80}$ \\
\hline $17.30($ core 3$)$ & & 18.80 & ${ }^{\mathrm{a}} 18.80$ \\
\hline $17.50 \bullet \leftarrow$ & $\leftarrow$ & $\bullet 19.00$ (core 2$)$ & ${ }^{\mathrm{a}} 19.00$ \\
\hline$\frac{17.50}{18.80}$ & & 19.10 (core 3$)$ & $a_{20.30}$ \\
\hline 19.60 & & 19.90 & $a_{21.10}$ \\
\hline 22.20 & & 22.25 & $a_{23.70}$ \\
\hline $23.00 \downarrow$ & & 23.00 & $\mathrm{~b}_{24.50}$ \\
\hline \multirow[t]{2}{*}{26.30} & & 26.30 & $b_{27.80}$ \\
\hline & 26.00 & \multirow[t]{2}{*}{29.00} & $b_{29.50}$ \\
\hline \multirow[t]{3}{*}{30.00} & 27.50 & & 31.50 \\
\hline & 28.00 & \multirow{18}{*}{$\begin{array}{l}30.75 \\
37.20\end{array}$} & $b_{32.00}$ \\
\hline & 34.50 & & $b_{38.50}$ \\
\hline $39.80 \downarrow$ & 36.00 & & $b_{41.30}$ \\
\hline 44.40 & 40.70 & & $\mathrm{~b}_{45.90}$ \\
\hline 58.50 & 55.50 & & ${ }^{b} 60.00$ \\
\hline 63.50 & 60.50 & & ${ }^{b} 65.00$ \\
\hline 69.00 & 66.50 & & $b_{70.50}$ \\
\hline $78.00 \downarrow$ & 76.00 & & b 79.50 \\
\hline 81.00 & $(82.90)=78.50$ & & b 82.50 \\
\hline 82.20 & $(84.15)=79.75$ & & $b_{83.70}$ \\
\hline 84.50 & $(87.25)=82.85$ & & b 86.00 \\
\hline 87.10 & $(90.00)=85.60$ & & $b_{88.60}$ \\
\hline $89.00 \downarrow$ & $(92.00)=87.60$ & & $\mathrm{~b}_{90.50}$ \\
\hline $93.95 \vee$ & 93.50 & & a95.45 \\
\hline 94.85 & 94.30 & & a96.35 \\
\hline 95.35 & 94.80 & & ${ }^{\mathrm{a}} 96.85$ \\
\hline 95.80 & 95.40 & & a97.30 \\
\hline 96.25 & 95.90 & & a97.75 \\
\hline $98.75 \downarrow$ & 98.20 & 100.75 & ${ }^{\mathrm{a}} 100.25$ \\
\hline 99.65 & 99.40 & 102.00 & ${ }^{\mathrm{a}} 101.15$ \\
\hline 100.55 & 100.40 & 102.90 & ${ }^{\mathrm{a}} 102.05$ \\
\hline \multirow{2}{*}{$101.05 \bullet \rightarrow$} & $\bullet 100.90 \bullet$ & 103.40 & $\mathrm{a}_{102.55}$ \\
\hline & 101.85 & 104.40 & ${ }^{\mathrm{a}} 103.50$ \\
\hline \multirow[t]{5}{*}{$103.70 ?$} & 103.35 & 105.90 & ${ }^{a} 105.00$ \\
\hline & $104.45 \downarrow$ & 106.95 & ${ }^{\mathrm{a}} 106.10$ \\
\hline & 106.20 & 108.75 & $\mathrm{a}_{107.85}$ \\
\hline & 108.40 & 110.15 & $\mathrm{a}_{110.05}$ \\
\hline & $110.50 \bullet \rightarrow$ & - 112.45 & $\mathrm{a}_{112.15}$ \\
\hline $112.50 \bullet$ & $\leftarrow$ & - 114.40 & $a_{114.10}$ \\
\hline 113.35 & & 114.95 & $a_{114.95}$ \\
\hline 117.00 | & & 117.40 & $a_{118.60}$ \\
\hline $119.00 \downarrow$ & 117.25 & & $b_{120.60}$ \\
\hline
\end{tabular}

Note: Underlined depths indicate switch points based on between-hole correlations.

${ }^{\text {a }}$ Correlation based on susceptibility data.

${ }^{b}$ From shipboard party correlation points. 
Table 3. Oxygen and carbon isotope data from Site 658 .

\begin{tabular}{|c|c|c|c|c|c|}
\hline \multirow{2}{*}{$\begin{array}{c}\begin{array}{c}\text { Core, section, } \\
\text { interval }(\mathrm{cm})\end{array} \\
658 \mathrm{~A}-1 \mathrm{H}-1,12-16\end{array}$} & \multirow{2}{*}{$\begin{array}{c}\begin{array}{c}\text { Depth } \\
\text { (c.d.- } \\
\text { mbsf) }\end{array} \\
0.24\end{array}$} & \multicolumn{2}{|c|}{$\begin{array}{c}\text { C. } \\
\text { wuellerstorfi }\end{array}$} & \multicolumn{2}{|c|}{$\delta^{18} \mathrm{O} \quad \delta^{\text {G. inflata }}$} \\
\hline & & 2.71 & 0.80 & 0.63 & 0.40 \\
\hline $658 \mathrm{~B}-2 \mathrm{H}-1,10-14$ & 0.72 & 2.69 & 0.96 & 1.09 & 0.68 \\
\hline $658 \mathrm{~A}-1 \mathrm{H}-1,70-75$ & 0.83 & 2.67 & 0.79 & 0.99 & 0.59 \\
\hline $658 \mathrm{~A}-1 \mathrm{H}-1,85-87$ & 0.96 & 2.67 & 0.86 & 1.06 & 0.64 \\
\hline $658 \mathrm{~A}-1 \mathrm{H}-1,102-104$ & 1.13 & 2.66 & 1.01 & 0.98 & 0.48 \\
\hline $658 \mathrm{~B}-2 \mathrm{H}-1,76-80$ & 1.38 & 2.81 & 0.58 & 1.14 & 0.29 \\
\hline $658 \mathrm{~B}-2 \mathrm{H}-1,102-104$ & 1.63 & 2.54 & 0.61 & 1.16 & 0.29 \\
\hline $658 \mathrm{~A}-1 \mathrm{H}-2,12-16$ & 1.74 & 2.85 & 0.65 & 1.25 & 0.19 \\
\hline $658 \mathrm{~B}-2 \mathrm{H}-2,20-24$ & 2.32 & 3.37 & 0.34 & 1.77 & 0.21 \\
\hline $658 \mathrm{~A}-1 \mathrm{H}-2,70-75$ & 2.33 & 3.50 & 0.57 & 1.74 & 0.21 \\
\hline $658 \mathrm{~A}-1 \mathrm{H}-2,91-93$ & 2.50 & 3.17 & 0.53 & 2.19 & 0.27 \\
\hline $658 \mathrm{~A}-1 \mathrm{H}-2,102-104$ & 2.61 & 3.58 & 0.48 & 2.14 & 0.05 \\
\hline $658 \mathrm{~B}-2 \mathrm{H}-2,95-99$ & 3.07 & 4.10 & 0.17 & 2.20 & 0.15 \\
\hline $658 \mathrm{~B}-2 \mathrm{H}-2,102-104$ & 3.13 & 3.72 & 0.27 & 2.25 & 0.14 \\
\hline $658 \mathrm{~A}-1 \mathrm{H}-3,12-16$ & 3.15 & 4.36 & 0.49 & 2.09 & 0.11 \\
\hline $658 \mathrm{~A}-1 \mathrm{H}-3,70-75$ & 3.68 & 4.17 & 0.45 & 2.11 & 0.27 \\
\hline $658 \mathrm{~B}-2 \mathrm{H}-3,20-24$ & 3.82 & 4.48 & 0.53 & 2.35 & 0.11 \\
\hline $658 \mathrm{~A}-1 \mathrm{H}-3,91-93$ & 3.86 & 4.01 & 0.49 & 2.23 & 0.32 \\
\hline $658 \mathrm{~A}-1 \mathrm{H}-3,102-104$ & 3.95 & 4.31 & 0.60 & 2.14 & 0.26 \\
\hline $658 \mathrm{~B}-2 \mathrm{H}-3,72-76$ & 4.33 & 4.28 & 0.52 & 1.97 & -0.29 \\
\hline $658 \mathrm{~A}-1 \mathrm{H}-4,12-16$ & 4.50 & 3.70 & 0.42 & 1.84 & 0.24 \\
\hline $658 \mathrm{~B}-2 \mathrm{H}-3,95-99$ & 4.57 & 4.39 & 0.49 & 2.19 & 0.10 \\
\hline $658 \mathrm{~B}-2 \mathrm{H}-3,102-104$ & 4.63 & 4.33 & 0.52 & 2.28 & 0.18 \\
\hline $658 \mathrm{~A}-1 \mathrm{H}-4,70-75$ & 5.04 & 4.02 & 0.57 & 1.89 & 0.47 \\
\hline $658 \mathrm{~A}-1 \mathrm{H}-4,80-82$ & 5.12 & 3.98 & 0.52 & 1.94 & 0.31 \\
\hline $658 \mathrm{~B}-2 \mathrm{H}-4,20-24$ & 5.32 & 4.19 & 0.57 & 2.26 & 0.16 \\
\hline $658 \mathrm{~B}-2 \mathrm{H}-4,95-99$ & 6.07 & 4.11 & 0.58 & 2.17 & 0.33 \\
\hline $658 \mathrm{~B}-2 \mathrm{H}-4,102-104$ & 6.13 & 3.90 & 0.52 & 1.79 & 0.43 \\
\hline $658 \mathrm{~B}-2 \mathrm{H}-5,20-24$ & 6.82 & 3.71 & 0.30 & 1.88 & 0.45 \\
\hline $658 \mathrm{~B}-2 \mathrm{H}-5,72-76$ & 7.33 & 3.93 & 0.69 & 2.01 & 0.40 \\
\hline $658 \mathrm{~B}-2 \mathrm{H}-5,95-99$ & 7.57 & 3.97 & 0.50 & 1.98 & 0.26 \\
\hline $658 \mathrm{~B}-2 \mathrm{H}-5,102-104$ & 7.63 & 4.02 & 0.48 & 1.99 & 0.27 \\
\hline $658 \mathrm{~B}-2 \mathrm{H}-6,50-54$ & 8.32 & 3.98 & 0.46 & 1.82 & 0.10 \\
\hline $658 \mathrm{~B}-2 \mathrm{H}-6,102-104$ & 8.83 & 3.18 & 0.16 & 1.63 & 0.50 \\
\hline $658 \mathrm{~B}-2 \mathrm{H}-6,128-132$ & 9.10 & 3.10 & 0.73 & 1.13 & 0.16 \\
\hline $658 \mathrm{~B}-2 \mathrm{H}-7,28-32$ & 9.60 & 3.58 & 0.47 & 1.39 & 0.46 \\
\hline $658 \mathrm{~B}-2 \mathrm{H}-7,38-40$ & 9.69 & 2.76 & 0.58 & 0.84 & 0.07 \\
\hline $658 \mathrm{~B}-3 \mathrm{H}-1,20-24$ & 10.32 & 3.47 & 0.63 & 1.44 & 0.49 \\
\hline $658 \mathrm{~B}-3 \mathrm{H}-1,102-104$ & 11.13 & 3.42 & 0.59 & 1.32 & 0.26 \\
\hline $658 \mathrm{~B}-3 \mathrm{H}-1,106-110$ & 11.18 & 3.30 & 0.55 & 1.31 & 0.21 \\
\hline $658 \mathrm{~B}-3 \mathrm{H}-2,20-24$ & 11.83 & 3.25 & 0.62 & 1.52 & 0.34 \\
\hline $658 \mathrm{~B}-3 \mathrm{H}-2,72-76$ & 12.34 & 3.20 & 0.47 & 1.21 & 0.12 \\
\hline $658 \mathrm{~A}-3 \mathrm{H}-1,12-16$ & 12.49 & 3.44 & 0.59 & 1.25 & 0.14 \\
\hline 658B-3H-2, 94-98 & 12.56 & 3.20 & 0.32 & 1.46 & 0.40 \\
\hline 658B-3H-2, 102-104 & 12.63 & 3.24 & 0.61 & 1.23 & 0.26 \\
\hline $658 \mathrm{~A}-3 \mathrm{H}-1,93-97$ & 13.30 & 3.14 & 0.15 & 1.28 & -0.01 \\
\hline $658 \mathrm{~A}-3 \mathrm{H}-1,102-104$ & 13.38 & 3.29 & 0.12 & 1.29 & 0.21 \\
\hline $658 \mathrm{~B}-3 \mathrm{H}-3,50-54$ & 13.62 & 3.04 & 0.35 & 0.97 & -0.21 \\
\hline $658 \mathrm{~A}-3 \mathrm{H}-2,12-16$ & 13.99 & 3.32 & 0.24 & 1.29 & 0.11 \\
\hline $658 \mathrm{~B}-3 \mathrm{H}-3,102-104$ & 14.13 & 3.52 & 0.18 & 1.21 & -0.01 \\
\hline $658 \mathrm{~A}-3 \mathrm{H}-2,93-97$ & 14.80 & 3.57 & 0.12 & 1.35 & 0.16 \\
\hline $658 \mathrm{~A}-3 \mathrm{H}-2,102-104$ & 14.88 & 3.06 & 0.05 & 1.57 & 0.28 \\
\hline $658 \mathrm{~A}-3 \mathrm{H}-3,12-16$ & 15.49 & 3.15 & -0.02 & 0.64 & 0.14 \\
\hline $658 \mathrm{~A}-3 \mathrm{H}-3,93-97$ & 16.30 & 2.65 & 0.57 & 0.76 & -0.15 \\
\hline $658 \mathrm{~A}-3 \mathrm{H}-3,102-104$ & 16.38 & 2.62 & 0.52 & 1.04 & 0.04 \\
\hline $658 \mathrm{~A}-3 \mathrm{H}-4,12-16$ & 16.99 & 2.91 & -0.02 & 0.84 & -0.24 \\
\hline $658 \mathrm{~A}-3 \mathrm{H}-4,95-99$ & 17.82 & 4.47 & 0.09 & 1.96 & -0.36 \\
\hline $658 \mathrm{~A}-3 \mathrm{H}-4,102-104$ & 17.87 & 4.33 & -0.08 & 2.05 & -0.45 \\
\hline $658 \mathrm{~A}-3 \mathrm{H}-5,12-16$ & 18.49 & 4.35 & 0.18 & 2.08 & -0.57 \\
\hline $658 \mathrm{~A}-3 \mathrm{H}-5,93-97$ & 19.30 & 4.09 & -0.02 & 2.06 & -0.53 \\
\hline $658 \mathrm{~A}-3 \mathrm{H}-5,102-\mathrm{I04}$ & 19.38 & 3.68 & -0.64 & 2.26 & -0.23 \\
\hline $658 \mathrm{~A}-3 \mathrm{H}-6,12-16$ & 19.99 & 4.05 & 0.03 & 2.16 & -0.58 \\
\hline $658 \mathrm{~B}-4 \mathrm{H}-1,126-130$ & 20.58 & 4.27 & 0.14 & 1.90 & -0.62 \\
\hline $658 \mathrm{~A}-3 \mathrm{H}-6,93-97$ & 20.80 & 4.08 & 0.11 & 1.81 & -0.39 \\
\hline $658 \mathrm{~A}-3 \mathrm{H}-6,102-104$ & 20.88 & 3.94 & 0.24 & 1.56 & -0.01 \\
\hline $658 \mathrm{~A}-3 \mathrm{H}-\mathrm{CC}, 16-21$ & 21.13 & 3.85 & 0.13 & 1.52 & -0.27 \\
\hline $658 \mathrm{~B}-4 \mathrm{H}-2,62-66$ & 21.59 & 3.78 & 0.11 & 1.83 & -0.43 \\
\hline $658 \mathrm{~A}-4 \mathrm{H}-1,20-24$ & 22.07 & 3.89 & 0.08 & 1.76 & -0.50 \\
\hline $658 \mathrm{~A}-4 \mathrm{H}-1,69-71$ & 22.55 & 3.83 & -0.18 & 1.63 & -0.59 \\
\hline $658 \mathrm{~A}-4 \mathrm{H}-1,70-75$ & 22.58 & 3.83 & 0.03 & 2.03 & -0.38 \\
\hline $658 \mathrm{~A}-4 \mathrm{H}-1,102-104$ & 22.79 & 3.93 & -0.09 & 2.08 & -0.24 \\
\hline $658 \mathrm{~A}-4 \mathrm{H}-2,12-16$ & 23.40 & 4.00 & 0.09 & 1.69 & -0.26 \\
\hline $658 \mathrm{~A}-4 \mathrm{H}-2,70-75$ & 23.99 & 3.96 & 0.16 & 1.65 & -0.03 \\
\hline $658 \mathrm{~A}-4 \mathrm{H}-2,93-97$ & 24.21 & 3.72 & 0.12 & 1.58 & 0.13 \\
\hline $658 \mathrm{~A}-4 \mathrm{H}-2,102-104$ & 24.29 & 3.46 & 0.44 & 1.41 & 0.09 \\
\hline $658 \mathrm{~A}-4 \mathrm{H}-3,12-16$ & 24.90 & 3.23 & 0.08 & 1.39 & -0.26 \\
\hline
\end{tabular}

Table 3 (continued).

\begin{tabular}{|c|c|c|c|c|c|}
\hline \multirow{2}{*}{$\begin{array}{c}\begin{array}{c}\text { Core, section, } \\
\text { interval (cm) }\end{array} \\
658 \mathrm{~A}-4 \mathrm{H}-3,70-75\end{array}$} & \multirow{2}{*}{$\begin{array}{l}\begin{array}{l}\text { Depth } \\
\text { (c.d.- } \\
\text { mbsf) }\end{array} \\
25.49\end{array}$} & \multicolumn{2}{|c|}{$\begin{array}{c}\text { C. } \\
\text { wuellerstorfi }\end{array}$} & \multicolumn{2}{|c|}{${ }_{8^{18} \mathrm{O}}^{\text {G. inflata }}{ }^{13} \mathrm{C}$} \\
\hline & & 3.27 & 0.19 & 1.81 & 0.26 \\
\hline $658 \mathrm{~A}-4 \mathrm{H}-3,93-97$ & 25.71 & & 0.48 & 1.55 & 0.01 \\
\hline $658 \mathrm{~A}-4 \mathrm{H}-3,102-104$ & 25.79 & 3.32 & 0.12 & 1.39 & 0.07 \\
\hline $658 \mathrm{~A}-4 \mathrm{H}-4,12-16$ & 26.40 & 2.85 & 0.30 & 1.06 & -0.08 \\
\hline $658 \mathrm{~A}-4 \mathrm{H}-4,70-75$ & 26.99 & 3.30 & 0.26 & 1.67 & -0.02 \\
\hline $658 \mathrm{~A}-4 \mathrm{H}-4,93-97$ & 27.21 & 3.14 & 0.14 & 1.31 & 0.01 \\
\hline $658 \mathrm{~A}-4 \mathrm{H}-4,102-104$ & 27.29 & 3.10 & 0.27 & 1.40 & 0.10 \\
\hline $658 \mathrm{~A}-4 \mathrm{H}-5,12-16$ & 27.90 & 3.10 & 0.34 & 1.28 & 0.37 \\
\hline $658 \mathrm{~A}-4 \mathrm{H}-5,70-75$ & 28.49 & 2.87 & 0.30 & 1.43 & 0.06 \\
\hline $658 \mathrm{~A}-4 \mathrm{H}-5,93-97$ & 28.71 & 2.77 & 0.26 & - & - \\
\hline $658 \mathrm{~A}-4 \mathrm{H}-5,102-104$ & 28.79 & 3.06 & 0.09 & 1.56 & -0.03 \\
\hline $658 \mathrm{~A}-4 \mathrm{H}-5,110-114$ & 28.88 & 3.00 & 0.17 & 1.36 & -0.03 \\
\hline $658 \mathrm{~A}-4 \mathrm{H}-6,12-16$ & 29.40 & 3.64 & -0.20 & 1.82 & -0.49 \\
\hline $658 \mathrm{~A}-4 \mathrm{H}-6,70-75$ & 29.99 & 4.05 & 0.01 & - & - \\
\hline $658 \mathrm{~A}-4 \mathrm{H}-6,93-97$ & 30.21 & 3.48 & -0.08 & 1.85 & -0.18 \\
\hline $658 \mathrm{~A}-4 \mathrm{H}-6,102-104$ & 30.29 & 3.45 & 0.01 & 1.88 & -0.22 \\
\hline $658 \mathrm{~A}-5 \mathrm{H}-1,15-19$ & 31.52 & 3.43 & -0.16 & 2.14 & -0.15 \\
\hline $658 \mathrm{~A}-5 \mathrm{H}-1,70-75$ & 32.08 & 3.02 & 0.20 & 1.19 & 0.10 \\
\hline $658 \mathrm{~A}-5 \mathrm{H}-1,93-95$ & 32.29 & 2.83 & -0.08 & 1.23 & -0.03 \\
\hline $658 \mathrm{~A}-5 \mathrm{H}-1,102-104$ & 32.38 & 2.80 & 0.15 & 1.08 & -0.13 \\
\hline $658 \mathrm{~A}-5 \mathrm{H}-2,12-16$ & 32.99 & 3.56 & 0.15 & 0.89 & -0.35 \\
\hline $658 \mathrm{~A}-5 \mathrm{H}-2,70-75$ & 33.58 & 2.65 & -0.41 & 1.27 & -0.39 \\
\hline $658 \mathrm{~A}-5 \mathrm{H}-2,93-95$ & 33.79 & 2.85 & -0.11 & 1.63 & -0.41 \\
\hline $658 \mathrm{~A}-5 \mathrm{H}-2,102-104$ & 33.88 & 3.36 & -0.11 & 1.62 & -0.29 \\
\hline $658 \mathrm{~A}-5 \mathrm{H}-2,106-110$ & 33.93 & 3.71 & 0.01 & 1.85 & -0.23 \\
\hline $658 \mathrm{~A}-5 \mathrm{H}-3,12-16$ & 34.49 & 3.70 & -0.11 & 1.88 & -0.52 \\
\hline $658 \mathrm{~A}-5 \mathrm{H}-3,70-75$ & 35.08 & 3.99 & 0.11 & 1.61 & -0.55 \\
\hline $658 \mathrm{~A}-5 \mathrm{H}-3,82-85$ & 35.19 & 3.93 & 0.16 & 1.97 & -0.50 \\
\hline $658 \mathrm{~A}-5 \mathrm{H}-3,102-104$ & 35.38 & 4.07 & 0.28 & 2.10 & -0.26 \\
\hline $658 \mathrm{~A}-5 \mathrm{H}-4,12-16$ & 35.99 & 3.99 & 0.22 & 1.83 & -0.44 \\
\hline $658 \mathrm{~A}-5 \mathrm{H}-4,70-75$ & 36.58 & 3.59 & -0.10 & 1.89 & -0.41 \\
\hline $658 \mathrm{~A}-5 \mathrm{H}-4,93-95$ & 36.79 & 3.83 & -0.04 & 2.02 & -0.33 \\
\hline $658 \mathrm{~A}-5 \mathrm{H}-4,102-104$ & 36.88 & 3.77 & -0.14 & 1.76 & -0.70 \\
\hline $658 \mathrm{~A}-5 \mathrm{H}-5,12-16$ & 37.49 & 4.05 & -0.35 & 2.68 & -0.17 \\
\hline $658 \mathrm{~A}-5 \mathrm{H}-5,70-75$ & 38.08 & 3.88 & -0.29 & 2.18 & -0.02 \\
\hline $658 \mathrm{~A}-5 \mathrm{H}-5,85-87$ & 38.21 & 3.82 & -0.33 & 2.56 & 0.29 \\
\hline $658 \mathrm{~A}-5 \mathrm{H}-5,102-104$ & 38.38 & 3.62 & -0.35 & 2.22 & 0.20 \\
\hline $658 \mathrm{~A}-5 \mathrm{H}-6,12-16$ & 38.99 & 3.87 & 0.29 & - & - \\
\hline $658 \mathrm{~A}-5 \mathrm{H}-6,70-75$ & 39.57 & 3.66 & 0.51 & 1.57 & 0.28 \\
\hline $658 \mathrm{~A}-5 \mathrm{H}-6,93-95$ & 39.79 & 3.73 & 0.47 & 1.85 & 0.40 \\
\hline $658 \mathrm{~A}-5 \mathrm{H}-6,102-104$ & 39.88 & 3.53 & 0.33 & 1.62 & 0.51 \\
\hline $658 \mathrm{~A}-5 \mathrm{H}-7,14-18$ & 40.51 & 3.25 & 0.48 & 1.36 & 0.29 \\
\hline $658 \mathrm{~A}-5 \mathrm{H}-7,25-30$ & 40.63 & 3.26 & 0.35 & 1.47 & 0.27 \\
\hline $658 \mathrm{~A}-5 \mathrm{H}-7,44-46$ & 40.80 & 3.34 & 0.36 & 1.34 & 0.25 \\
\hline $658 \mathrm{~A}-5 \mathrm{H}-7,50-54$ & 40.84 & 3.36 & 0.38 & 1.73 & 0.21 \\
\hline $8 \mathrm{~A}-6 \mathrm{H}-1,65-69$ & 41.5 & & 0.30 & 1.88 & 0.45 \\
\hline $658 \mathrm{~A}-6 \mathrm{H}-1,70-75$ & 41.58 & 3.74 & 0.31 & 1.74 & 0.31 \\
\hline $658 \mathrm{~A}-6 \mathrm{H}-1,102-104$ & 41.8 & 3.76 & -0.02 & 2.10 & 0.20 \\
\hline $658 \mathrm{~A}-6 \mathrm{H}-2,12-16$ & 42.49 & 3.65 & 0.43 & 2.04 & 0.51 \\
\hline $658 \mathrm{~A}-6 \mathrm{H}-2,70-75$ & 43.0 & 3.35 & 0.08 & 1.86 & 0.29 \\
\hline 658A-6H-2, 94-98 & 43.27 & - & - & 2.13 & 0.50 \\
\hline $658 \mathrm{~A}-6 \mathrm{H}-2,102-104$ & 43.34 & 3.50 & -0.28 & 2.03 & 0.55 \\
\hline $658 \mathrm{~A}-6 \mathrm{H}-2,106-110$ & 43.39 & - & - & 2.12 & 0.43 \\
\hline $658 \mathrm{~A}-6 \mathrm{H}-3,70-75$ & 44.53 & 3.24 & 0.26 & 1.60 & 0.36 \\
\hline $658 \mathrm{~A}-6 \mathrm{H}-3,94-98$ & 44.77 & 2.94 & 0.37 & 1.17 & 0.02 \\
\hline $658 \mathrm{~A}-6 \mathrm{H}-3,102-104$ & 44.84 & 3.05 & -0.42 & 1.16 & -0.06 \\
\hline $658 \mathrm{~A}-6 \mathrm{H}-5,70-75$ & 46.05 & - & - & 1.45 & 0.46 \\
\hline $658 \mathrm{~A}-6 \mathrm{H}-5,83-87$ & 46.17 & 2.95 & 0.37 & 1.47 & 0.18 \\
\hline $658 \mathrm{~A}-6 \mathrm{H}-5,102-104$ & 46.3 & 2.97 & 0.26 & 1.33 & 0.27 \\
\hline $8 \mathrm{~A}-6 \mathrm{H}-6,12-16$ & 49.9 & 2.76 & 0.54 & 1.09 & 0.05 \\
\hline $8 \mathrm{~A}-6 \mathrm{H}-6,70-75$ & 47.55 & 2.58 & 0.03 & 1.02 & 0.07 \\
\hline $658 \mathrm{~A}-6 \mathrm{H}-6,83-87$ & 47.67 & 2.75 & - & 0.88 & -0.04 \\
\hline $658 \mathrm{~A}-6 \mathrm{H}-6,102-104$ & 47.85 & 2.54 & 0.03 & 1.20 & -0.15 \\
\hline $8 \mathrm{~A}-6 \mathrm{H}-6,106-110$ & 47.90 & - & - & 1.13 & 0.06 \\
\hline $658 \mathrm{~A}-6 \mathrm{H}-7,12-16$ & & - & - & 1.14 & -0.13 \\
\hline $658 \mathrm{~A}-6 \mathrm{H}-7,70-75$ & 49.04 & 3.00 & 0.03 & 0.49 & -0.27 \\
\hline $658 \mathrm{~A}-6 \mathrm{H}-7,98-100$ & 49. & 2.68 & -0.22 & 1.89 & -0.37 \\
\hline $8 \mathrm{~A}-6 \mathrm{H}-9,5-9$ & 49.67 & - & - & 1.66 & -0.23 \\
\hline $8 \mathrm{~A}-6 \mathrm{H}-9,15-20$ & 49.77 & 3.50 & -0.53 & 1.69 & -0.40 \\
\hline $658 \mathrm{~A}-6 \mathrm{H}-9,20-24$ & 49.82 & - & - & 1.69 & -0.32 \\
\hline A-6H-9, 33-35 & & - & - & 1.89 & -0.38 \\
\hline $8 \mathrm{~A}-7 \mathrm{H}-1,70-75$ & & 3.75 & -0.33 & 2.53 & 0.10 \\
\hline $658 \mathrm{~A}-7 \mathrm{H}-1,76-78$ & & 4.54 & 0.04 & 2.46 & 0.04 \\
\hline $658 \mathrm{~A}-7 \mathrm{H}-1,102-104$ & & 4.32 & 0.09 & 2.36 & -0.03 \\
\hline $658 \mathrm{~A}-7 \mathrm{H}-2,12-16$ & 51.99 & 4.02 & -0.07 & 2.32 & -0.08 \\
\hline $658 \mathrm{~A}-7 \mathrm{H}-2,70-75$ & 52.58 & 3.86 & -0.18 & 2.24 & -0.10 \\
\hline
\end{tabular}


Table 3 (continued).

\begin{tabular}{|c|c|c|c|c|c|}
\hline $\begin{array}{l}\text { Core, section, } \\
\text { interval }(\mathrm{cm})\end{array}$ & $\begin{array}{l}\text { Depth } \\
\text { (c.d.- } \\
\text { mbsf) }\end{array}$ & \multicolumn{2}{|c|}{$\begin{array}{c}C . \\
\text { wuellerstorfi }\end{array}$} & \multicolumn{2}{|c|}{${ }^{18} \mathrm{O}$. inflata ${ }^{13} \mathrm{C}$} \\
\hline $658 \mathrm{~A}-7 \mathrm{H}-2,85-89$ & 52.72 & 4.05 & -0.29 & 2.38 & 0.03 \\
\hline $658 \mathrm{~A}-7 \mathrm{H}-2,102-104$ & 52.88 & 4.07 & -0.16 & 2.52 & 0.07 \\
\hline $658 \mathrm{~A}-7 \mathrm{H}-3,12-16$ & 53.49 & 4.17 & -0.07 & 2.70 & 0.40 \\
\hline $658 \mathrm{~A}-7 \mathrm{H}-3,70-75$ & 54.07 & 4.02 & 0.14 & 2.11 & 0.36 \\
\hline $658 \mathrm{~A}-7 \mathrm{H}-3,85-89$ & 54.22 & 3.81 & 0.16 & 2.15 & 0.26 \\
\hline $658 \mathrm{~A}-7 \mathrm{H}-3,102-104$ & 54.38 & 3.84 & -0.03 & 1.47 & -0.15 \\
\hline $658 \mathrm{~A}-7 \mathrm{H}-4,12-16$ & 54.99 & 3.71 & 0.15 & 2.03 & 0.48 \\
\hline $658 \mathrm{~A}-7 \mathrm{H}-4,70-75$ & 55.58 & 3.57 & 0.35 & 1.80 & 0.46 \\
\hline $658 \mathrm{~A}-7 \mathrm{H}-4,85-89$ & 55.72 & 3.79 & 0.35 & 1.68 & 0.33 \\
\hline $658 \mathrm{~A}-7 \mathrm{H}-4,102-104$ & 55.88 & 3.59 & 0.34 & 1.98 & 0.40 \\
\hline 658B-7H-6, 94-98 & 56.46 & 3.93 & 0.42 & 1.99 & 0.56 \\
\hline $658 \mathrm{~A}-7 \mathrm{H}-5,12-16$ & 56.49 & 3.82 & 0.39 & 1.99 & 0.39 \\
\hline $658 \mathrm{~A}-7 \mathrm{H}-5,28-30$ & 56.64 & 3.92 & 0.34 & 1.73 & 0.41 \\
\hline $658 \mathrm{~B}-7 \mathrm{H}-7,18-22$ & 57.47 & 3.70 & 0.54 & 1.46 & 0.45 \\
\hline $658 \mathrm{~B}-8 \mathrm{H}-1,37-41$ & 58.41 & 3.27 & -0.04 & 1.58 & 0.24 \\
\hline $658 \mathrm{~B}-8 \mathrm{H}-2,50-54$ & 60.55 & 2.85 & 0.98 & 1.23 & 0.69 \\
\hline $658 \mathrm{~A}-8 \mathrm{H}-1,70-75$ & 60.58 & 2.44 & 0.68 & 1.01 & 0.51 \\
\hline $658 \mathrm{~A}-8 \mathrm{H}-1,83-87$ & 60.70 & 2.56 & 0.50 & 1.35 & 0.61 \\
\hline $658 \mathrm{~A}-8 \mathrm{H}-1,102-104$ & 60.88 & 2.58 & 0.63 & 0.92 & 0.57 \\
\hline $658 \mathrm{~A}-8 \mathrm{H}-2,12-16$ & 61.49 & 2.91 & 0.52 & 1.36 & 0.42 \\
\hline $658 \mathrm{~A}-8 \mathrm{H}-2,70-75$ & 62.07 & 3.16 & 0.42 & 1.03 & -0.32 \\
\hline $658 \mathrm{~A}-8 \mathrm{H}-2,83-87$ & 62.20 & 3.30 & 0.49 & 1.32 & -0.17 \\
\hline $658 \mathrm{~A}-8 \mathrm{H}-2,102-104$ & 62.38 & 3.39 & 0.27 & 0.88 & -0.77 \\
\hline $658 \mathrm{~A}-8 \mathrm{H}-2,106-110$ & 62.43 & 3.45 & 0.46 & 1.33 & -0.31 \\
\hline $658 \mathrm{~A}-8 \mathrm{H}-2,144-146$ & 62.80 & 2.99 & 0.23 & 2.11 & -0.27 \\
\hline $658 \mathrm{~A}-8 \mathrm{H}-3,26-28$ & 63.12 & - & - & 1.64 & -0.23 \\
\hline $658 \mathrm{~A}-8 \mathrm{H}-3,55-57$ & 63.41 & 4.31 & -0.25 & 0.46 & -0.16 \\
\hline $658 \mathrm{~A}-8 \mathrm{H}-3,70-75$ & 63.58 & 4.15 & -0.40 & 2.63 & -0.03 \\
\hline $658 \mathrm{~A}-8 \mathrm{H}-3,83-87$ & 63.70 & 4.49 & -0.12 & 2.78 & 0.07 \\
\hline $658 \mathrm{~A}-8 \mathrm{H}-3,89-91$ & 63.75 & 4.62 & -0.01 & 2.80 & 0.25 \\
\hline $658 \mathrm{~A}-8 \mathrm{H}-3,102-104$ & 63.88 & 4.56 & -0.11 & 2.75 & 0.20 \\
\hline $658 \mathrm{~A}-8 \mathrm{H}-3,133-135$ & 64.19 & 4.76 & -0.11 & 2.62 & 0.13 \\
\hline $658 \mathrm{~A}-8 \mathrm{H}-4,12-16$ & 64.49 & 4.63 & -0.11 & 2.78 & 0.15 \\
\hline $658 \mathrm{~A}-8 \mathrm{H}-4,70-75$ & 65.08 & 4.53 & -0.12 & 2.63 & 0.16 \\
\hline $658 \mathrm{~A}-8 \mathrm{H}-4,82-86$ & 65.19 & 4.33 & -0.20 & 2.51 & 0.18 \\
\hline $658 \mathrm{~A}-8 \mathrm{H}-4,102-104$ & 65.38 & 4.40 & 0.22 & 2.14 & 0.14 \\
\hline $658 \mathrm{~A}-8 \mathrm{H}-4,106-110$ & 65.43 & 4.55 & -0.07 & 2.39 & 0.14 \\
\hline $658 \mathrm{~A}-8 \mathrm{H}-5,12-16$ & 65.99 & 4.04 & -0.04 & 2.24 & -0.15 \\
\hline $658 \mathrm{~A}-8 \mathrm{H}-5,70-75$ & 66.58 & 3.95 & -0.16 & 2.17 & 0.02 \\
\hline $658 \mathrm{~A}-8 \mathrm{H}-5,83-87$ & 66.70 & 3.63 & -0.28 & 2.38 & 0.08 \\
\hline $658 \mathrm{~A}-8 \mathrm{H}-5,102-104$ & 66.88 & 4.03 & -0.13 & 2.43 & 0.06 \\
\hline $658 \mathrm{~A}-8 \mathrm{H}-6,12-16$ & 67.49 & 4.04 & -0.28 & 2.32 & 0.18 \\
\hline $658 \mathrm{~A}-8 \mathrm{H}-6,70-75$ & 68.07 & 4.19 & 0.01 & 2.19 & 0.01 \\
\hline $658 \mathrm{~A}-8 \mathrm{H}-6,85-98$ & 68.22 & 4.07 & -0.14 & 2.38 & 0.15 \\
\hline $658 \mathrm{~A}-8 \mathrm{H}-6,102-104$ & 68.38 & 4.11 & 0.08 & 2.02 & 0.12 \\
\hline $658 \mathrm{~A}-8 \mathrm{H}-6,106-110$ & 68.43 & 4.18 & 0.11 & 2.31 & 0.20 \\
\hline $658 \mathrm{~A}-9 \mathrm{H}-1,70-75$ & 70.07 & 3.22 & 0.05 & 1.76 & 0.38 \\
\hline $658 \mathrm{~A}-9 \mathrm{H}-1,81-85$ & 70.18 & 3.13 & 0.01 & 1.80 & 0.22 \\
\hline $658 \mathrm{~A}-9 \mathrm{H}-1,96-98$ & 70.32 & 3.64 & 0.06 & 1.51 & 0.03 \\
\hline $658 \mathrm{~A}-9 \mathrm{H}-2,12-16$ & 70.93 & 3.40 & 0.71 & 1.79 & 0.83 \\
\hline $658 \mathrm{~A}-9 \mathrm{H}-2,60-65$ & 71.33 & 3.18 & 0.40 & 1.50 & 0.70 \\
\hline $658 \mathrm{~A}-9 \mathrm{H}-2,99-103$ & 71.64 & 3.33 & 0.32 & 1.72 & 0.70 \\
\hline $658 \mathrm{~A}-9 \mathrm{H}-2,115-117$ & 71.95 & 3.24 & 0.70 & 1.65 & 0.75 \\
\hline $658 \mathrm{~A}-9 \mathrm{H}-3,12-16$ & 72.27 & 3.17 & 0.86 & 1.61 & 0.92 \\
\hline $658 \mathrm{~A}-9 \mathrm{H}-3,70-75$ & 72.71 & 3.13 & 0.62 & 1.53 & 0.81 \\
\hline $658 \mathrm{~A}-9 \mathrm{H}-3,131-133$ & 73.16 & 3.14 & 0.54 & 1.51 & 0.82 \\
\hline $658 \mathrm{~A}-9 \mathrm{H}-4,12-16$ & 73.32 & 2.99 & 0.73 & - & - \\
\hline $658 \mathrm{~A}-9 \mathrm{H}-4,70-75$ & 73.90 & 3.19 & 0.78 & 1.31 & 0.69 \\
\hline $658 \mathrm{~A}-9 \mathrm{H}-4,91-95$ & 74.11 & 3.15 & 0.55 & 1.55 & 0.83 \\
\hline $658 \mathrm{~A}-9 \mathrm{H}-4,102-104$ & 74.37 & 3.42 & 0.51 & 1.25 & 0.50 \\
\hline $658 \mathrm{~A}-9 \mathrm{H}-5,12-16$ & 74.82 & 3.14 & 0.54 & 1.42 & 0.75 \\
\hline $658 \mathrm{~A}-9 \mathrm{H}-5,70-75$ & 75.40 & 3.29 & 0.63 & 1.27 & 0.52 \\
\hline $658 \mathrm{~A}-9 \mathrm{H}-5,91-95$ & 75.61 & 3.03 & 0.59 & 1.45 & 0.58 \\
\hline $658 \mathrm{~A}-9 \mathrm{H}-5,102-104$ & 75.87 & 3.39 & 0.23 & 1.22 & 0.48 \\
\hline $658 \mathrm{~A}-9 \mathrm{H}-5,106-110$ & 75.92 & 3.43 & 0.51 & 1.30 & 0.68 \\
\hline $658 \mathrm{~A}-9 \mathrm{H}-6,12-16$ & 76.32 & 4.38 & 0.13 & 2.54 & -0.10 \\
\hline $658 \mathrm{~A}-9 \mathrm{H}-6,70-75$ & 76.90 & 3.58 & 0.82 & 1.61 & 0.57 \\
\hline $658 \mathrm{~A}-9 \mathrm{H}-6,81-85$ & 77.01 & 3.70 & 0.62 & 1.63 & 0.62 \\
\hline $658 \mathrm{~A}-9 \mathrm{H}-6,102-104$ & 77.21 & 3.77 & 0.84 & 1.46 & 0.38 \\
\hline $658 \mathrm{~A}-9 \mathrm{H}-6,140-144$ & 77.60 & 3.66 & 0.59 & 1.27 & 0.40 \\
\hline $658 \mathrm{~A}-10 \mathrm{H}-1,12-16$ & 78.99 & 3.29 & 0.66 & 1.41 & 0.50 \\
\hline $658 \mathrm{~A}-10 \mathrm{H}-1,70-75$ & 79.57 & - & - & 1.64 & 0.47 \\
\hline $658 \mathrm{~A}-10 \mathrm{H}-1,81-85$ & 79.68 & 3.57 & 0.40 & 1.57 & 0.40 \\
\hline $658 \mathrm{~A}-10 \mathrm{H}-1,104-106$ & 79.90 & 3.51 & 0.45 & 1.74 & 0.28 \\
\hline $658 \mathrm{~A}-10 \mathrm{H}-2,12-16$ & 80.49 & 3.58 & 0.22 & 1.77 & -0.01 \\
\hline $658 \mathrm{~A}-10 \mathrm{H}-2,70-75$ & 81.07 & 4.18 & 0.39 & 2.15 & 0.32 \\
\hline $658 \mathrm{~A}-10 \mathrm{H}-2,81-85$ & 81.18 & 3.77 & 0.24 & 1.61 & 0.18 \\
\hline $658 \mathrm{~A}-10 \mathrm{H}-2,102-104$ & 81.38 & 4.18 & 0.12 & 1.84 & 0.22 \\
\hline
\end{tabular}

Table 3 (continued).

\begin{tabular}{|c|c|c|c|c|c|}
\hline $\begin{array}{l}\text { Core, section, } \\
\text { interval }(\mathrm{cm})\end{array}$ & $\begin{array}{l}\text { Depth } \\
\text { (c.d.- } \\
\text { mbsf) }\end{array}$ & \multicolumn{2}{|c|}{$\begin{array}{c}C . \\
\text { wuellerstorfi }\end{array}$} & \multicolumn{2}{|c|}{$\delta^{18} \mathrm{O}{ }^{\text {G. inflata }} \delta^{13} \mathrm{C}$} \\
\hline $658 \mathrm{~A}-10 \mathrm{H}-2,106-110$ & 81.43 & 4.36 & 0.12 & 2.27 & 0.31 \\
\hline $658 \mathrm{~A}-10 \mathrm{H}-3,12-16$ & 81.99 & 4.12 & 0.20 & 1.88 & 0.15 \\
\hline $658 \mathrm{~A}-10 \mathrm{H}-3,70-75$ & 82.57 & 4.07 & 0.15 & 1.90 & 0.23 \\
\hline $658 \mathrm{~A}-10 \mathrm{H}-3,81-85$ & 82.68 & 4.06 & 0.25 & 1.92 & -0.04 \\
\hline $658 \mathrm{~A}-10 \mathrm{H}-3,102-104$ & 82.88 & 3.71 & 0.16 & 1.33 & -0.30 \\
\hline $658 \mathrm{~A}-10 \mathrm{H}-4,12-16$ & 83.49 & 3.48 & -0.18 & 1.84 & 0.23 \\
\hline $658 \mathrm{~A}-10 \mathrm{H}-4,70-75$ & 84.07 & 4.00 & 0.04 & 1.74 & 0.17 \\
\hline $658 \mathrm{~A}-10 \mathrm{H}-4,81-85$ & 84.18 & 3.29 & -0.64 & 1.58 & 0.25 \\
\hline $658 \mathrm{~A}-10 \mathrm{H}-4,102-104$ & 84.38 & 3.22 & 0.05 & 1.18 & -0.04 \\
\hline $658 \mathrm{~A}-10 \mathrm{H}-5,12-16$ & 84.92 & 3.37 & 0.42 & - & - \\
\hline $658 \mathrm{~A}-10 \mathrm{H}-5,70-75$ & 85.49 & 2.96 & 0.38 & 1.66 & 0.94 \\
\hline $658 \mathrm{~A}-10 \mathrm{H}-5,84-88$ & 85.62 & 3.02 & 0.57 & 1.30 & 0.54 \\
\hline $658 \mathrm{~A}-10 \mathrm{H}-5,102-104$ & 85.79 & 3.14 & 0.46 & 1.15 & 0.64 \\
\hline $658 \mathrm{~A}-10 \mathrm{H}-5,106-110$ & 85.84 & 3.00 & 0.51 & 1.41 & 0.60 \\
\hline $658 \mathrm{~A}-10 \mathrm{H}-6,60-65$ & 86.81 & 2.84 & 0.43 & 1.43 & 0.18 \\
\hline $658 \mathrm{~A}-10 \mathrm{H}-6,65-69$ & 86.86 & 2.71 & 0.41 & 1.20 & 0.08 \\
\hline $658 \mathrm{~A}-10 \mathrm{H}-6,74-76$ & 86.94 & - & - & 1.14 & -0.04 \\
\hline $658 \mathrm{~A}-11 \mathrm{H}-1,70-74$ & 89.04 & 3.63 & 0.53 & 1.44 & -0.07 \\
\hline $658 \mathrm{~A}-11 \mathrm{H}-2,12-16$ & 89.20 & 3.57 & 0.40 & 1.63 & 0.17 \\
\hline $658 \mathrm{~A}-11 \mathrm{H}-3,12-16$ & 90.04 & 3.60 & 0.35 & 1.74 & 0.35 \\
\hline 658A-11H-3, 70-74 & 90.58 & 3.39 & 0.14 & 1.56 & 0.23 \\
\hline $658 \mathrm{~A}-11 \mathrm{H}-3,104-106$ & 90.90 & - & - & 1.12 & -0.15 \\
\hline $658 \mathrm{~A}-11 \mathrm{H}-3,106-110$ & 90.93 & 3.38 & 0.67 & 1.30 & -0.13 \\
\hline $658 \mathrm{~A}-11 \mathrm{H}-4,12-16$ & 91.36 & 3.07 & 0.29 & 1.20 & 0.10 \\
\hline $658 \mathrm{~A}-11 \mathrm{H}-4,70-74$ & 91.94 & 3.01 & 0.58 & 1.30 & 0.30 \\
\hline $658 \mathrm{~A}-11 \mathrm{H}-4,102-104$ & 92.27 & 2.99 & 0.28 & 1.75 & 0.14 \\
\hline $658 \mathrm{~A}-11 \mathrm{H}-5,12-16$ & 92.79 & 2.97 & -0.39 & 1.82 & 0.16 \\
\hline $658 \mathrm{~A}-11 \mathrm{H}-5,70-74$ & 93.37 & 4.84 & 0.18 & 2.42 & -0.07 \\
\hline $658 \mathrm{~A}-11 \mathrm{H}-5,102-104$ & 93.69 & 4.56 & -0.05 & 2.17 & -0.38 \\
\hline $658 \mathrm{~A}-11 \mathrm{H}-5,106-110$ & 93.74 & 4.69 & 0.23 & 2.53 & -0.03 \\
\hline $658 \mathrm{~A}-11 \mathrm{H}-6,12-16$ & 94.20 & 4.15 & 0.03 & 2.03 & -0.10 \\
\hline $658 \mathrm{~A}-11 \mathrm{H}-6,70-74$ & 94.77 & 3.61 & -0.73 & 2.45 & 0.34 \\
\hline $658 \mathrm{~A}-11 \mathrm{H}-6,102-104$ & 95.08 & - & - & 1.48 & 0.51 \\
\hline $658 \mathrm{~A}-11 \mathrm{H}-6,106-110$ & 95.13 & 3.33 & 0.21 & 1.40 & 0.18 \\
\hline $658 \mathrm{~A}-11 \mathrm{H}-7,70-74$ & 96.16 & 3.61 & -0.04 & 1.93 & 0.53 \\
\hline $658 \mathrm{~A}-11 \mathrm{H}-7,102-104$ & 96.41 & 3.56 & -0.47 & 1.91 & 0.10 \\
\hline $658 \mathrm{~A}-11 \mathrm{H}-8,12-16$ & 96.99 & 3.36 & -0.13 & 1.32 & 0.10 \\
\hline $658 \mathrm{~A}-11 \mathrm{H}-8,68-72$ & 97.55 & 3.59 & -0.14 & 1.75 & -0.16 \\
\hline $658 \mathrm{~A}-11 \mathrm{H}-8,75-77$ & 97.61 & 3.49 & 0.03 & 1.71 & -0.20 \\
\hline $658 \mathrm{~A}-12 \mathrm{H}-1,12-16$ & 97.98 & 2.86 & -0.19 & 1.17 & -0.01 \\
\hline $658 \mathrm{~A}-12 \mathrm{H}-1,70-74$ & 98.51 & 2.97 & -0.44 & - & - \\
\hline $658 \mathrm{~A}-12 \mathrm{H}-1,100-102$ & 98.79 & - & - & 1.09 & -0.16 \\
\hline $658 \mathrm{~A}-12 \mathrm{H}-2,12-16$ & 98.93 & - & - & 1.90 & 0.16 \\
\hline $658 \mathrm{~A}-12 \mathrm{H}-2,70-74$ & 99.23 & 3.92 & -0.84 & 2.02 & 0.34 \\
\hline $658 \mathrm{~A}-12 \mathrm{H}-2,100-102$ & 99.51 & 3.82 & -0.35 & 1.95 & 0.13 \\
\hline $658 \mathrm{~A}-12 \mathrm{H}-3,70-74$ & 100.08 & 3.20 & 0.25 & 1.86 & 0.37 \\
\hline $658 \mathrm{~A}-12 \mathrm{H}-3,100-102$ & 100.56 & 3.25 & -0.18 & 1.67 & 0.31 \\
\hline $658 \mathrm{~A}-12 \mathrm{H}-4,70-74$ & 101.72 & - & - & 1.81 & 0.47 \\
\hline $658 \mathrm{~A}-12 \mathrm{H}-4,107-109$ & 102.04 & 2.91 & 0.32 & 1.52 & 0.31 \\
\hline $658 \mathrm{~A}-12 \mathrm{H}-5,120-122$ & 103.17 & 3.25 & -0.14 & 1.81 & 0.08 \\
\hline $658 \mathrm{~A}-12 \mathrm{H}-6,70-74$ & 104.13 & 3.86 & 0.02 & 2.33 & 0.08 \\
\hline $658 \mathrm{~A}-12 \mathrm{H}-6,102-104$ & 104.43 & 3.81 & -0.14 & 1.88 & 0.12 \\
\hline $658 \mathrm{~A}-12 \mathrm{H}-7,70-74$ & 105.57 & 3.28 & 0.35 & 1.43 & 0.54 \\
\hline $658 \mathrm{~A}-12 \mathrm{H}-7,102-104$ & 105.81 & 3.22 & 0.46 & 1.08 & 0.58 \\
\hline $658 \mathrm{~A}-12 \mathrm{H}-8,70-74$ & 107.00 & 2.99 & 0.27 & 0.94 & 0.25 \\
\hline $658 \mathrm{~A}-12 \mathrm{H}-8,80-82$ & 107.08 & 3.21 & -0.03 & - & - \\
\hline $658 \mathrm{~A}-13 \mathrm{H}-1,12-16$ & 107.49 & 3.10 & 0.17 & - & - \\
\hline $658 \mathrm{~A}-13 \mathrm{H}-1,72-85$ & 108.09 & 3.32 & -0.32 & 1.91 & 0.10 \\
\hline $658 \mathrm{~A}-13 \mathrm{H}-1,102-104$ & 108.38 & 3.55 & 0.23 & 2.07 & 0.10 \\
\hline $658 \mathrm{~A}-13 \mathrm{H}-2,72-75$ & 109.02 & 3.53 & 0.01 & 1.46 & 0.13 \\
\hline $658 \mathrm{~A}-13 \mathrm{H}-2,107-109$ & 109.12 & 3.26 & 0.05 & 1.41 & 0.07 \\
\hline $658 \mathrm{~A}-13 \mathrm{H}-3,12-16$ & 109.57 & 3.21 & 0.07 & - & - \\
\hline $658 \mathrm{~A}-13 \mathrm{H}-3,72-75$ & 110.17 & 3.22 & 0.24 & 1.49 & 0.56 \\
\hline $658 \mathrm{~A}-13 \mathrm{H}-3,102-104$ & 110.46 & 3.32 & -0.28 & 1.67 & 0.48 \\
\hline $658 \mathrm{~A}-13 \mathrm{H}-4,72-75$ & 111.67 & 3.39 & 0.06 & 1.36 & 0.24 \\
\hline $658 \mathrm{~A}-13 \mathrm{H}-4,102-104$ & 111.96 & 3.57 & 0.29 & 1.64 & 0.38 \\
\hline $658 \mathrm{~A}-13 \mathrm{H}-5,56-59$ & 112.92 & 2.92 & 0.99 & 1.72 & 0.74 \\
\hline $658 \mathrm{~A}-13 \mathrm{H}-5,102-104$ & 113.21 & 3.15 & 0.45 & 1.85 & 0.59 \\
\hline $658 \mathrm{~A}-13 \mathrm{H}-6,68-71$ & 114.27 & 3.02 & 0.31 & 1.50 & 0.51 \\
\hline $658 \mathrm{~A}-13 \mathrm{H}-6,102-104$ & 114.50 & - & - & 1.60 & 0.46 \\
\hline $658 \mathrm{~A}-13 \mathrm{H}-7,12-16$ & 115.01 & 2.79 & 0.57 & - & - \\
\hline $658 \mathrm{~A}-13 \mathrm{H}-7,72-75$ & 115.53 & 3.05 & 0.31 & 1.45 & 0.41 \\
\hline $658 \mathrm{~A}-13 \mathrm{H}-7,102-104$ & 115.82 & 2.93 & 0.45 & 1.73 & 0.49 \\
\hline $658 \mathrm{~A}-13 \mathrm{H}-8,72-75$ & 116.79 & 2.95 & 0.22 & 1.45 & 0.26 \\
\hline $658 \mathrm{~A}-14 \mathrm{H}-1,70-74$ & 117.65 & 3.70 & - & 2.23 & 0.50 \\
\hline $658 \mathrm{~A}-14 \mathrm{H}-1,98-100$ & 117.83 & 3.32 & 0.04 & 2.24 & 0.67 \\
\hline $658 \mathrm{~A}-14 \mathrm{H}-2,98-100$ & 118.88 & 3.10 & - & 2.29 & 0.80 \\
\hline $658 \mathrm{~A}-14 \mathrm{H}-3,70-74$ & 119.84 & 3.17 & 0.23 & 1.86 & 0.73 \\
\hline
\end{tabular}


Table 3 (continued).

\begin{tabular}{|c|c|c|c|c|c|}
\hline $\begin{array}{l}\text { Core, section, } \\
\text { interval }(\mathrm{cm})\end{array}$ & $\begin{array}{l}\text { Depth } \\
\text { (c.d.- } \\
\text { mbsf) }\end{array}$ & \multicolumn{2}{|c|}{$\begin{array}{c}C . \\
\text { wuellerstorf }\end{array}$} & \multicolumn{2}{|c|}{$\begin{array}{c}\text { G. inflata } \\
\delta^{18} \mathrm{O}{ }^{13} \mathrm{C}\end{array}$} \\
\hline $58 \mathrm{~A}-14 \mathrm{H}-3,98-100$ & 120.11 & 2.74 & 0.27 & 1.94 & 0.69 \\
\hline $658 \mathrm{~A}-14 \mathrm{H}-4,12-16$ & 120.67 & 2.79 & 0.45 & 2.00 & 0.76 \\
\hline $658 \mathrm{~A}-14 \mathrm{H}-4,67-71$ & 121.13 & 2.97 & 0.51 & 2.04 & 0.75 \\
\hline $658 \mathrm{~A}-14 \mathrm{H}-4,98-100$ & 121.43 & 2.77 & 0.19 & 1.45 & 0.22 \\
\hline $658 \mathrm{~A}-14 \mathrm{H}-5,72-76$ & 122.59 & 3.28 & -0.52 & 2.08 & 0.26 \\
\hline $658 \mathrm{~A}-14 \mathrm{H}-5,98-100$ & 122.84 & 2.90 & -0.26 & 2.23 & 0.43 \\
\hline $658 \mathrm{~A}-14 \mathrm{H}-6,12-16$ & 123.47 & 2.81 & 0.16 & 1.53 & 0.48 \\
\hline $658 \mathrm{~A}-14 \mathrm{H}-6,70-74$ & 124.01 & 2.76 & 0.42 & 1.63 & 0.76 \\
\hline $658 \mathrm{~A}-14 \mathrm{H}-6,98-100$ & 124.28 & 2.68 & 0.21 & 1.54 & 0.77 \\
\hline $658 \mathrm{~A}-14 \mathrm{H}-7,66-68$ & 125.36 & 2.86 & 0.64 & 1.80 & 0.60 \\
\hline $658 \mathrm{~A}-14 \mathrm{H}-7,96-98$ & 125.66 & 2.98 & 0.01 & 1.67 & 0.62 \\
\hline $658 \mathrm{~A}-14 \mathrm{H}-8,12-16$ & 126.26 & 2.78 & -0.15 & 1.73 & 0.44 \\
\hline $658 \mathrm{~A}-14 \mathrm{H}-8,68-82$ & 126.71 & 2.64 & 0.23 & 1.71 & 0.34 \\
\hline $658 \mathrm{~A}-15 \mathrm{H}-1,67-71$ & 127.04 & 3.16 & 0.59 & 1.73 & 0.81 \\
\hline $658 \mathrm{~A}-15 \mathrm{H}-1,83-85$ & 127.19 & - & - & 1.80 & 0.81 \\
\hline $658 \mathrm{~A}-15 \mathrm{H}-2,12-16$ & 127.47 & 2.59 & 0.62 & 1.36 & 0.63 \\
\hline $658 \mathrm{~A}-15 \mathrm{H}-2,72-76$ & 127.98 & 2.65 & 0.63 & 1.45 & 0.85 \\
\hline $658 \mathrm{~A}-15 \mathrm{H}-2,96-98$ & 128.21 & 2.82 & 0.53 & 1.44 & 0.87 \\
\hline $658 \mathrm{~A}-15 \mathrm{H}-3,72-75$ & 129.08 & 2.59 & -0.08 & 1.60 & 0.72 \\
\hline $658 \mathrm{~A}-15 \mathrm{H}-3,102-104$ & 129.47 & 2.74 & 0.23 & 1.52 & 0.58 \\
\hline $658 \mathrm{~A}-15 \mathrm{H}-4,12-16$ & 129.90 & 2.15 & 0.09 & 1.92 & 0.91 \\
\hline $658 \mathrm{~A}-15 \mathrm{H}-4,72-75$ & 130.42 & 2.52 & 0.65 & 1.78 & 0.62 \\
\hline $658 \mathrm{~A}-15 \mathrm{H}-4,100-102$ & 130.63 & - & - & 1.55 & 0.50 \\
\hline $658 \mathrm{~A}-15 \mathrm{H}-4,130-134$ & 130.83 & - & - & 1.91 & 0.88 \\
\hline 658A-15H-5, 75-78 & 131.73 & 3.17 & 0.28 & 1.90 & 0.72 \\
\hline $658 \mathrm{~A}-15 \mathrm{H}-5,102-104$ & 131.99 & 3.36 & 0.30 & 1.72 & 0.54 \\
\hline $658 \mathrm{~A}-15 \mathrm{H}-5,131-135$ & 132.29 & 3.17 & 0.14 & 2.09 & 0.7 \\
\hline $658 \mathrm{~A}-15 \mathrm{H}-6,72-75$ & 132.99 & - & - & 1.86 & 0.65 \\
\hline $658 \mathrm{~A}-15 \mathrm{H}-6,102-104$ & 133.28 & 3.44 & 0.44 & 1.76 & 0.53 \\
\hline $658 \mathrm{~A}-15 \mathrm{H}-7,72-75$ & 134.40 & 3.66 & 0.09 & 2.00 & 0.14 \\
\hline $658 \mathrm{~A}-15 \mathrm{H}-7,84-86$ & 134.51 & 3.82 & -0.04 & 2.28 & 0.47 \\
\hline $658 \mathrm{~A}-15 \mathrm{H}-8,75-78$ & 136.01 & 3.67 & -0.20 & 2.16 & 0.37 \\
\hline $658 \mathrm{~A}-15 \mathrm{H}-8,82-84$ & 136.07 & 3.56 & -0.33 & 2.14 & 0.41 \\
\hline $658 \mathrm{~A}-16 \mathrm{H}-1,102-104$ & 136.75 & 3.07 & 1.47 & - & - \\
\hline $658 \mathrm{~A}-16 \mathrm{H}-2,102-104$ & 138.18 & 3.09 & 0.33 & - & - \\
\hline $658 \mathrm{~A}-\mathrm{C}-\mathrm{C} \mathrm{C}$ & 138. & 3.02 & -0.33 & - & - \\
\hline $658 \mathrm{~A}-16 \mathrm{H}-3,70-74$ & 139.37 & 3.16 & 0.06 & - & - \\
\hline $2-104$ & 139.68 & 2.86 & -0.25 & - & - \\
\hline $658 \mathrm{~A}-16 \mathrm{H}-4,11$ & 140.8 & 2.85 & -0.09 & - & - \\
\hline $658 \mathrm{~A}$ & 141. & 2.83 & 0.48 & - & - \\
\hline $658 \mathrm{~A}-16 \mathrm{H}-5,70-74$ & 142.37 & 3.02 & -0.14 & - & - \\
\hline$-16 \mathrm{H}-6,70-74$ & 143.8 & 4.00 & -0.50 & - & - \\
\hline $658 \mathrm{~A}-16 \mathrm{H}-6,102-104$ & 144. & 3.46 & -0.57 & - & - \\
\hline 658 & 144. & 3.89 & -0.36 & - & - \\
\hline $12-16$ & 144.7 & 3.47 & 0.1 & - & - \\
\hline & 145. & 3.02 & -0.19 & - & - \\
\hline 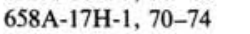 & 146.07 & 2.66 & 0.21 & - & - \\
\hline-102 & 146. & 2.99 & 0.0 & - & - \\
\hline 74 & 147. & 3.01 & -0.46 & - & - \\
\hline $104-106$ & 147. & 3.23 & -0.08 & - & - \\
\hline $658 \mathrm{~A}-\mathrm{C}-\mathrm{C} \mathrm{C}$ & 149. & 2.91 & -1.04 & - & - \\
\hline $658 \mathrm{~A}-17 \mathrm{H}$ & 149.3 & 2.77 & -0.48 & - & - \\
\hline $658 \mathrm{~A}-17 \mathrm{H}-4,56-60$ & 150.12 & 3.13 & 0.28 & - & - \\
\hline $\begin{array}{l}\text { Core, section, } \\
\text { interval }(\mathrm{cm})\end{array}$ & $\begin{array}{l}\text { Depth } \\
\text { (c.d.- } \\
\text { mbsf) }\end{array}$ & & $\begin{array}{l}\text { storfi } \\
\delta^{13} \mathrm{C}\end{array}$ & $\begin{array}{c}U . \\
\text { pere- } \\
\text { grina } \\
\delta^{18} \mathrm{O}\end{array}$ & $\begin{array}{c}U . \\
\text { auber- } \\
\text { iana } \\
\delta^{13} \mathrm{C}\end{array}$ \\
\hline $8 \mathrm{~A}-17 \mathrm{H}-5,70-74$ & 151.73 & 2.71 & 0.08 & 3.73 & 3.70 \\
\hline $0-102$ & 1.99 & - & - & 3.61 & 3.71 \\
\hline $658 \mathrm{~A}-17 \mathrm{H}-5,116-120$ & 152.1 & - & - & - & 3.70 \\
\hline $658 \mathrm{~A}-17 \mathrm{H}-6,12-14$ & 152.5 & - & - & 4.16 & - \\
\hline $658 \mathrm{~A}-17 \mathrm{H}-6,40-44$ & 152.7 & 3.19 & 0.01 & 4.21 & - \\
\hline $658 \mathrm{~A}-17 \mathrm{H}-6,64-66$ & 153.0 & - & - & 4.10 & - \\
\hline $658 \mathrm{~A}-19 \mathrm{X}-1,70-74$ & 155.77 & - & - & 4.21 & - \\
\hline $658 \mathrm{~A}-19 \mathrm{X}-1,105-107$ & 156.11 & - & - & 3.77 & 3.82 \\
\hline $658 \mathrm{~A}-19 \mathrm{X}-2,70-74$ & 156.8 & 3.15 & -0.45 & 4.31 & 4.28 \\
\hline $658 \mathrm{~A}-19 \mathrm{X}-2,87-89$ & 157.0 & - & - & 4.30 & - \\
\hline $658 \mathrm{~A}-19 \mathrm{X}-3,70-74$ & 157.6 & 2.96 & & 4.11 & - \\
\hline $658 \mathrm{~A}-19 \mathrm{X}-3,102-104$ & 158. & 3.06 & -0.40 & 3.88 & - \\
\hline $658 \mathrm{~A}-19 \mathrm{X}-4,40-44$ & 158.6 & 2.40 & 0.51 & 3.75 & - \\
\hline $658 \mathrm{~A}-19 \mathrm{X}-4,102-104$ & 159.14 & - & - & 3.60 & - \\
\hline $658 \mathrm{~A}-19 \mathrm{X}-5,12-16$ & 149.65 & 2.82 & 0.51 & 3.41 & - \\
\hline $658 \mathrm{~A}-19 \mathrm{X}-5,66-70$ & & - & - & 3.62 & - \\
\hline $658 \mathrm{~A}-19 \mathrm{X}-5,102-104$ & 160.50 & 2.63 & 0.06 & - & \\
\hline $658 \mathrm{~A}-19 \mathrm{X}-6,130-134$ & 161.79 & 3.30 & -0.35 & 3.99 & - \\
\hline $658 \mathrm{~A}-19 \mathrm{X}-7,66-70$ & 162.54 & - & - & 4.65 & - \\
\hline $58 \mathrm{~A}-19 \mathrm{X}-7,102-$ & 162.82 & 94 & 0.61 & & \\
\hline
\end{tabular}

Table 3 (continued).

\begin{tabular}{|c|c|c|c|c|c|}
\hline $\begin{array}{l}\text { Core, section, } \\
\text { interval }(\mathrm{cm})\end{array}$ & $\begin{array}{l}\text { Depth } \\
\text { (c.d.- } \\
\text { mbsf) }\end{array}$ & $\begin{array}{l}\text { wuel } \\
\delta^{18} \mathrm{O}\end{array}$ & ${ }^{\text {storfi }}$ & $\begin{array}{c}U . \\
\text { pere- } \\
\text { grina } \\
{ }^{18} \mathrm{O}\end{array}$ & $\begin{array}{c}U . \\
\text { auber- } \\
\text { iana } \\
\delta^{13} \mathrm{C}\end{array}$ \\
\hline $658 \mathrm{~A}-19 \mathrm{X}-\mathrm{CC}, 10-14$ & 163.70 & 3.64 & -0.68 & - & \\
\hline $658 \mathrm{~A}-20 \mathrm{X}-1,12-16$ & 164.69 & 3.05 & 0.16 & 3.89 & 3.68 \\
\hline $658 \mathrm{~A}-20 \mathrm{X}-1,61-65$ & 165.18 & 2.68 & 0.35 & - & - \\
\hline $658 \mathrm{~A}-20 \mathrm{X}-1,110-112$ & 165.46 & 2.43 & 0.23 & - & 3.61 \\
\hline $658 \mathrm{~A}-20 \mathrm{X}-2,12-16$ & 166.29 & 2.72 & 0.41 & - & - \\
\hline $658 \mathrm{~A}-20 \mathrm{X}-2,70-74$ & 166.77 & 2.59 & 0.45 & 3.48 & 3.40 \\
\hline $658 \mathrm{~A}-20 \mathrm{X}-2,102-104$ & 167.08 & 2.57 & 0.26 & - & 3.37 \\
\hline $658 \mathrm{~A}-20 \mathrm{X}-2,130-134$ & 167.37 & 2.73 & 0.21 & - & - \\
\hline $658 \mathrm{~A}-20 \mathrm{X}-3,70-74$ & 168.24 & 2.99 & -0.10 & 3.79 & 3.74 \\
\hline $658 \mathrm{~A}-20 \mathrm{X}-3,102-104$ & 168.55 & 2.98 & -0.04 & - & 3.81 \\
\hline 658A-20X-6, 70-74 & 169.45 & 3.19 & 0.31 & 4.18 & 4.08 \\
\hline $658 \mathrm{~A}-20 \mathrm{X}-6,90-92$ & 169.64 & - & - & 3.99 & 4.12 \\
\hline $658 \mathrm{~A}-20 \mathrm{X}-7,70-74$ & 170.30 & 3.64 & 0.05 & 4.39 & 4.40 \\
\hline $658 \mathrm{~A}-20 \mathrm{X}-8,115-117$ & 171.62 & - & - & 3.95 & - \\
\hline $658 \mathrm{~A}-20 \mathrm{X}-9,12-16$ & 171.99 & - & - & 3.60 & - \\
\hline $658 \mathrm{~A}-20 \mathrm{X}-9,70-74$ & 172.30 & - & - & 3.91 & - \\
\hline $658 \mathrm{~A}-20 \mathrm{X}-9,89-91$ & 172.48 & - & - & 3.98 & - \\
\hline $658 \mathrm{~A}-20 \mathrm{X}-9,98-100$ & 172.57 & - & - & 3.79 & - \\
\hline $658 \mathrm{~A}-21 \mathrm{X}-1,50-54$ & 174.51 & 2.92 & 0.39 & 3.68 & - \\
\hline $658 \mathrm{~A}-21 \mathrm{X}-1,70-74$ & 174.71 & 2.67 & 0.32 & 3.54 & - \\
\hline $658 \mathrm{~A}-21 \mathrm{X}-1,114-116$ & 175.12 & 2.65 & 0.23 & 3.42 & 3.42 \\
\hline $658 \mathrm{~A}-21 \mathrm{X}-2,60-64$ & 176.03 & 2.64 & 0.31 & 3.36 & - \\
\hline $658 \mathrm{~A}-21 \mathrm{X}-2,80-82$ & 176.22 & 2.48 & 0.09 & 3.37 & - \\
\hline $658 \mathrm{~A}-21 \mathrm{X}-4,12-16$ & 176.72 & 2.71 & 0.40 & 3.45 & 3.49 \\
\hline $658 \mathrm{~A}-21 \mathrm{X}-4,66-70$ & 177.26 & 2.63 & 0.56 & 3.41 & - \\
\hline $658 \mathrm{~A}-21 \mathrm{X}-4,102-104$ & 177.45 & 2.73 & 0.68 & 3.42 & 3.36 \\
\hline $658 \mathrm{~A}-21 \mathrm{X}-5,65-65$ & 178.36 & 2.81 & 0.25 & 3.57 & 3.56 \\
\hline $658 \mathrm{~A}-21 \mathrm{X}-5,117-119$ & 178.71 & 2.96 & 0.25 & 3.39 & - \\
\hline $658 \mathrm{~A}-21 \mathrm{X}-6,58-62$ & 179.60 & 3.05 & -0.03 & 3.88 & 3.76 \\
\hline $658 \mathrm{~A}-21 \mathrm{X}-6,78-80$ & 179.79 & 3.06 & -0.12 & - & 3.78 \\
\hline$-21 X-8,12-16$ & 180.48 & 3.39 & -0.17 & 4.08 & 4.16 \\
\hline $658 \mathrm{~A}-21 \mathrm{X}-8,104-106$ & 181.39 & - & - & 4.41 & 4.40 \\
\hline 658A-22X-1, 103-105 & 183.89 & 2.96 & 0.02 & - & - \\
\hline $658 \mathrm{~A}-22 \mathrm{X}-2,12-16$ & 184.58 & 3.12 & 0.02 & 3.78 & - \\
\hline $658 \mathrm{~A}-22 \mathrm{X}-2,64-68$ & 185.10 & 2.84 & 0.17 & 3.57 & 3.56 \\
\hline $658 \mathrm{~A}-22 \mathrm{X}-2,94-96$ & 185.39 & 2.65 & 0.28 & 3.51 & 3.48 \\
\hline $658 \mathrm{~A}-22 \mathrm{X}-3,50-54$ & 185.96 & 2.64 & 0.35 & - & 3.46 \\
\hline $658 \mathrm{~A}-22 \mathrm{X}-3,103-105$ & 186.23 & 2.60 & 0.41 & - & 3.46 \\
\hline $658 \mathrm{~A}-22 \mathrm{X}-5,60-64$ & 187.43 & 2.58 & 0.10 & - & 3.53 \\
\hline $658 \mathrm{~A}-22 \mathrm{X}-5,96-98$ & 187.75 & 2.65 & -0.35 & - & 3.22 \\
\hline $658 \mathrm{~A}-22 \mathrm{X}-6,12-16$ & 188.27 & 2.79 & 0.52 & - & 3.26 \\
\hline $658 \mathrm{~A}-22 \mathrm{X}-6,70-74$ & 188.75 & 2.75 & 0.58 & 3.50 & 3.19 \\
\hline $658 \mathrm{~A}-22 \mathrm{X}-6,113-115$ & 189.01 & 2.63 & 0.22 & - & 3.25 \\
\hline $658 \mathrm{~A}-22 \mathrm{X}-7,56-60$ & 189.90 & 2.90 & 0.33 & 3.42 & 3.45 \\
\hline $658 \mathrm{~A}-22 \mathrm{X}-7,104-106$ & 190.25 & 2.75 & 0.18 & 3.42 & - \\
\hline $658 \mathrm{~A}-23 \mathrm{X}-1,70-74$ & 193.75 & 2.76 & 0.28 & 3.30 & 3.26 \\
\hline $658 \mathrm{~A}-23 \mathrm{X}-1,102-104$ & 194.08 & 2.61 & 0.45 & 2.87 & - \\
\hline $658 \mathrm{~A}-23 \mathrm{X}-2,65-69$ & 194.89 & 2.61 & 0.46 & 3.49 & 3.54 \\
\hline $658 \mathrm{~A}-23 \mathrm{X}-2,102-104$ & 195.19 & 2.62 & 0.53 & 3.40 & - \\
\hline$-23 X-3,61-65$ & 196. & 2.95 & 0.32 & 3.52 & 3.73 \\
\hline $658 \mathrm{~A}-23 \mathrm{X}-3,102-104$ & 196.33 & 2.98 & 0.02 & 3.68 & - \\
\hline $658 \mathrm{~A}-23 \mathrm{X}-4,131-133$ & 197. & 3.10 & -0.08 & 3.77 & - \\
\hline $658 \mathrm{~A}-23 \mathrm{X}-5,83-87$ & 8.05 & - & - & 3.66 & - \\
\hline A-23X-7, $53-55$ & 198.5 & - & - & 3.47 & - \\
\hline $658 \mathrm{~A}-23 \mathrm{X}-8,4-8$ & 9.04 & 2.85 & 0.01 & 3.69 & 3.65 \\
\hline$-23 X-9,70-74$ & & 2.73 & 0.0 & 3.56 & 3.51 \\
\hline $658 \mathrm{~A}-23 \mathrm{X}-9,102-104$ & 25 & 2.62 & 0.06 & 3.55 & - \\
\hline$-23 X-9,120-124$ & 2 & 2.49 & -0.01 & 3.53 & 3.41 \\
\hline$-24 X-1,10-14$ & 7 & 3.02 & 0.14 & 3.53 & - \\
\hline$A-24 X-2,102-10$ & & 2.81 & -0.20 & 3.65 & - \\
\hline A-24X-2, 133-137 & 1 & 2.76 & 0.86 & 3.59 & 3.63 \\
\hline - & & - & - & 3.61 & - \\
\hline$-24 X-3,102-104$ & & - & - & - & 3.95 \\
\hline 658 & & - & - & & - \\
\hline$-24 X-4,98-100$ & 34 & 3.15 & -0.10 & 3.95 & 3.98 \\
\hline $24 x-563-67$ & & 2.86 & & - & - \\
\hline$-24 X-5,102-104$ & & 2.64 & & - & 2.86 \\
\hline$-24 X-6,70-74$ & & 2.52 & -0.10 & - & 3.06 \\
\hline $24 \mathrm{X}-6,103-105$ & & 2.45 & 0.14 & - & 2.90 \\
\hline $24 X-7,83-87$ & & 2.57 & & - & 3.22 \\
\hline $2-104$ & 209. & 2.47 & 0.38 & 3.37 & - \\
\hline & & - & - & - & 3.54 \\
\hline $24 \mathrm{X}-9,31-33$ & & - & - & 3.58 & 3.61 \\
\hline & & 2.44 & & - & 3.34 \\
\hline $658 \mathrm{~A}-25 \mathrm{X}-1,70-74$ & 212.65 & 3.20 & -0.10 & - & 3.55 \\
\hline & 212.86 & 2.90 & -0.15 & 3.93 & 3.81 \\
\hline $658 \mathrm{~A}-25 \mathrm{X}-2,70-74$ & 213.95 & - & - & 3.98 & \\
\hline
\end{tabular}


Table 3 (continued).

\begin{tabular}{|c|c|c|c|c|c|}
\hline $\begin{array}{l}\text { Core, section, } \\
\text { interval }(\mathrm{cm})\end{array}$ & $\begin{array}{l}\text { Depth } \\
\text { (c.d.- } \\
\text { mbsf) }\end{array}$ & $\begin{array}{c}\text { wuell } \\
\delta^{18} \mathrm{O}\end{array}$ & $\begin{array}{l}\text { storfi } \\
\delta^{13} \mathrm{C}\end{array}$ & $\begin{array}{c}U . \\
\text { pere- } \\
\text { grina } \\
\delta^{18} \mathrm{O}\end{array}$ & $\begin{array}{c}U . \\
\text { auber } \\
\text { iana } \\
\delta^{13} \mathrm{C}\end{array}$ \\
\hline $658 \mathrm{~A}-25 \mathrm{X}-2,102-104$ & 214.25 & 3.12 & -0.22 & 3.89 & 3.90 \\
\hline $658 \mathrm{~A}-25 \mathrm{X}-3,70-74$ & 215.29 & - & - & - & 3.85 \\
\hline $658 \mathrm{~A}-25 \mathrm{X}-3,102-104$ & 215.60 & 3.07 & -0.30 & - & 3.79 \\
\hline $658 \mathrm{~A}-25 \mathrm{X}-4,70-74$ & 216.53 & 2.86 & -0.01 & 3.50 & 3.72 \\
\hline $658 \mathrm{~A}-25 \mathrm{X}-5,98-100$ & 217.81 & 2.54 & 0.26 & - & - \\
\hline $658 \mathrm{~A}-25 \mathrm{X}-6,69-73$ & 218.46 & 2.60 & 0.08 & - & - \\
\hline $658 A-25 X-6,88-90$ & 218.58 & 2.55 & -0.19 & - & - \\
\hline $658 \mathrm{~A}-25 \mathrm{X}-7,70-74$ & 219.35 & 2.71 & -0.04 & - & - \\
\hline $658 \mathrm{~A}-25 \mathrm{X}-7,88-90$ & 219.52 & 2.77 & -0.30 & - & - \\
\hline $658 \mathrm{~A}-26 \mathrm{X}-1,46-50$ & 222.03 & 2.70 & 0.16 & - & - \\
\hline $658 A-26 X-1,67-69$ & 222.23 & 2.65 & 0.19 & - & - \\
\hline $658 \mathrm{~A}-26 \mathrm{X}-2,12-16$ & 222.41 & 2.56 & 0.48 & - & - \\
\hline $658 \mathrm{~A}-26 \mathrm{X}-2,79-83$ & 222.69 & 2.68 & 0.33 & - & - \\
\hline $658 \mathrm{~A}-26 \mathrm{X}-2,104-106$ & 222.93 & 2.36 & 0.07 & - & - \\
\hline $658 A-26 X-3,66-70$ & 223.47 & 2.67 & 0.03 & - & - \\
\hline $658 \mathrm{~A}-26 \mathrm{X}-3,101-103$ & 223.72 & 2.62 & 0.37 & - & - \\
\hline $658 \mathrm{~A}-26 \mathrm{X}-4,54-58$ & 224.46 & 2.84 & 0.22 & - & - \\
\hline $658 A-26 X-4,94-96$ & 224.73 & 2.75 & 0.37 & - & - \\
\hline $658 \mathrm{~A}-26 \mathrm{X}-5,54-58$ & 225.54 & 3.03 & 0.06 & - & - \\
\hline $658 \mathrm{~A}-26 \mathrm{X}-5,132-135$ & 226.31 & 2.89 & 0.01 & - & - \\
\hline $658 \mathrm{~A}-26 \mathrm{X}-6,59-63$ & 226.97 & 3.04 & 0.01 & - & - \\
\hline $658 \mathrm{~A}-26 \mathrm{X}-6,92-94$ & 227.13 & 2.79 & 0.04 & - & - \\
\hline $658 \mathrm{~A}-26 \mathrm{X}-7,12-16$ & 227.32 & 2.97 & -0.12 & - & - \\
\hline $658 \mathrm{~A}-26 \mathrm{X}-7,69-73$ & 227.61 & 2.94 & -0.31 & - & - \\
\hline $658 \mathrm{~A}-26 \mathrm{X}-7,100-102$ & 227.85 & 2.96 & -0.32 & - & - \\
\hline $658 \mathrm{~A}-26 \mathrm{X}-8,55-59$ & 228.70 & 2.94 & -1.04 & - & - \\
\hline $658 \mathrm{~A}-26 \mathrm{X}-8,79-81$ & 228.93 & 2.76 & 0.20 & - & - \\
\hline $658 A-26 X-9,66-70$ & 229.63 & 2.31 & 0.30 & - & - \\
\hline $658 A-26 X-9,100-102$ & 229.94 & 2.37 & 0.43 & - & - \\
\hline $658 \mathrm{~A}-27 \mathrm{X}-1,70-74$ & 231.74 & 2.49 & 0.51 & 一 & - \\
\hline $658 A-27 X-2,12-16$ & 232.39 & 2.73 & 0.49 & - & - \\
\hline $658 \mathrm{~A}-27 \mathrm{X}-2,70-74$ & 232.89 & 2.44 & 0.56 & - & - \\
\hline $658 \mathrm{~A}-27 \mathrm{X}-2,98-100$ & 233.10 & 2.45 & 0.54 & - & - \\
\hline $658 A-27 X-3,63-67$ & 233.98 & 2.59 & 0.10 & - & - \\
\hline $658 \mathrm{~A}-27 \mathrm{X}-4,59-63$ & 234.94 & 2.50 & 0.32 & - & - \\
\hline $658 \mathrm{~A}-27 \mathrm{X}-4,100-102$ & 235.33 & 2.59 & 0.23 & - & 3.21 \\
\hline $658 \mathrm{~A}-27 \mathrm{X}-5,70-74$ & 236.07 & - & - & 3.26 & 3.26 \\
\hline $658 \mathrm{~A}-27 \mathrm{X}-5,102-104$ & 236.38 & 2.72 & 0.40 & 3.15 & 3.08 \\
\hline $658 A-27 X-6,12-16$ & 236.98 & 2.70 & -0.08 & 3.04 & 3.09 \\
\hline $658 \mathrm{~A}-27 \mathrm{X}-6,70-74$ & 237.53 & 2.72 & 0.21 & 2.94 & 3.20 \\
\hline $658 A-27 X-6,88-90$ & 237.71 & 2.44 & 0.44 & 3.46 & 3.48 \\
\hline $658 \mathrm{~A}-27 \mathrm{X}-7,12-16$ & 237.82 & 2.70 & 0.09 & 3.48 & 3.49 \\
\hline $658 \mathrm{~A}-27 \mathrm{X}-7,70-74$ & 238.40 & 2.79 & -0.13 & 3.58 & 3.68 \\
\hline $658 A-27 X-7,86-88$ & 238.55 & 2.72 & 0.33 & 3.39 & 3.40 \\
\hline $658 \mathrm{~A}-27 \mathrm{X}-\mathrm{CC}, 20-24$ & 239.03 & 2.91 & 0.23 & 3.66 & 3.68 \\
\hline $658 \mathrm{~A}-28 \mathrm{X}-1,10-14$ & 240.67 & - & - & 3.72 & - \\
\hline $658 \mathrm{~A}-28 \mathrm{X}-1,70-75$ & 241.28 & 一 & - & 3.88 & 3.84 \\
\hline $658 \mathrm{~A}-28 \mathrm{X}-1,96-100$ & 241.53 & - & - & 3.83 & 3.85 \\
\hline $658 \mathrm{~A}-28 \mathrm{X}-1,102-104$ & 241.58 & - & - & - & 3.79 \\
\hline $658 \mathrm{~A}-28 \mathrm{X}-2,12-16$ & 242.19 & - & - & 3.67 & 3.70 \\
\hline $658 \mathrm{~A}-28 \mathrm{X}-2,70-75$ & 242.77 & - & - & - & 3.45 \\
\hline $658 A-28 X-2,96-100$ & 243.03 & - & - & 3.46 & 3.50 \\
\hline $658 \mathrm{~A}-28 \mathrm{X}-2,102-104$ & 243.08 & 2.60 & 0.02 & 3.41 & 3.54 \\
\hline $658 \mathrm{~A}-28 \mathrm{X}-3,70-75$ & 244.02 & 1.92 & -0.74 & 2.83 & 3.37 \\
\hline $658 \mathrm{~A}-28 \mathrm{X}-3,96-100$ & 244.47 & 2.60 & -0.52 & - & - \\
\hline $658 \mathrm{~A}-28 \mathrm{X}-3,102-104$ & 244.52 & - & - & 3.55 & 3.43 \\
\hline $658 \mathrm{~A}-28 \mathrm{X}-4,70-75$ & 245.72 & - & - & 3.54 & 3.55 \\
\hline $658 \mathrm{~A}-28 \mathrm{X}-4,96-100$ & 245.97 & - & - & 3.59 & 3.58 \\
\hline $658 \mathrm{~A}-28 \mathrm{X}-4,102-104$ & 246.02 & 2.78 & 0.10 & 3.42 & 3.41 \\
\hline $658 \mathrm{~A}-28 \mathrm{X}-5,70-75$ & 247.22 & - & - & 3.67 & 3.70 \\
\hline $658 \mathrm{~A}-28 \mathrm{X}-5,100-102$ & 247.50 & 2.89 & 0.05 & 3.50 & 3.66 \\
\hline $658 \mathrm{~A}-28 \mathrm{X}-6,67-71$ & 248.45 & 2.78 & -0.07 & 3.54 & 3.62 \\
\hline $658 A-28 X-6,102-104$ & 248.77 & 2.87 & -0.13 & 3.64 & 3.73 \\
\hline $658 \mathrm{~A}-29 \mathrm{X}-1,25-27$ & 250.31 & 2.55 & -0.07 & 3.27 & 3.46 \\
\hline $658 \mathrm{~A}-29 \mathrm{X}-2,74-78$ & 251.10 & - & - & 3.45 & 3.50 \\
\hline $658 \mathrm{~A}-29 \mathrm{X}-2,102-104$ & 251.37 & 2.68 & -0.14 & 3.58 & 3.50 \\
\hline $658 A-29 X-3,72-76$ & 252.41 & 2.37 & 0.37 & 3.16 & 3.09 \\
\hline $658 A-29 X-4,102-104$ & 253.92 & 2.30 & 0.23 & - & - \\
\hline $658 A-29 X-5,72-76$ & 255.13 & 2.38 & 0.44 & - & - \\
\hline $658 \mathrm{~A}-29 \mathrm{X}-5,102-104$ & 255.42 & 2.47 & 0.34 & - & - \\
\hline $658 A-29 X-6,72-76$ & 256.30 & 2.56 & -0.11 & - & - \\
\hline $658 \mathrm{~A}-29 \mathrm{X}-6,102-104$ & 256.63 & 2.68 & 0.13 & - & - \\
\hline $658 \mathrm{~A}-29 \mathrm{X}-7,102-104$ & 257.88 & 2.85 & 0.25 & 3.37 & 3.29 \\
\hline $658 \mathrm{~A}-29 \mathrm{X}-8,46-48$ & 258.76 & - & - & 3.38 & 3.45 \\
\hline $658 \mathrm{~A}-29 \mathrm{X}-\mathrm{CC}, 6-10$ & 258.85 & 2.61 & 0.23 & 3.30 & 3.37 \\
\hline $658 \mathrm{~A}-30 \mathrm{X}-1,68-72$ & 260.25 & 2.66 & 0.09 & 3.61 & 3.63 \\
\hline
\end{tabular}

Table 3 (continued).

\begin{tabular}{lccccc}
\hline \multicolumn{1}{c}{$\begin{array}{c}\text { Core, section, } \\
\text { interval (cm) }\end{array}$} & $\begin{array}{c}\text { Depth } \\
\text { (c.d.- } \\
\text { mbsf) }\end{array}$ & $\begin{array}{c}\text { wuellerstorfi } \\
\delta^{18} \text { O }\end{array}$ & $\delta^{13} \mathrm{C}$ & $\begin{array}{c}\text { grina } \\
\delta^{18} \mathrm{O}\end{array}$ & $\begin{array}{c}\text { Uuber- } \\
\text { iana } \\
\delta^{13} \mathrm{C}\end{array}$ \\
\hline 658A-30X-1, 102-104 & 260.54 & 2.23 & -0.51 & 3.51 & 3.45 \\
658A-30X-3, 70-74 & 261.81 & 2.28 & -0.87 & 3.42 & 3.33 \\
658A-30X-3, 102-104 & 262.12 & 2.30 & -0.31 & 3.20 & 3.31 \\
658A-30X-4, 70-74 & 263.13 & - & - & - & 3.33 \\
658A-30X-4, 102-104 & 263.54 & - & - & 3.15 & 3.25 \\
658A-30X-5, 70-74 & 264.39 & - & - & 3.40 & 3.61 \\
658A-30X-5, 102-104 & 264.70 & - & - & 3.41 & 3.48 \\
658A-30X-6, 70-74 & 265.75 & - & - & 3.32 & 3.28 \\
658A-30X-6, 102-104 & 266.06 & - & - & 3.15 & - \\
658A-31X-1,70-74 & 269.74 & - & - & - & 3.12 \\
658A-31X-1, 102-104 & 270.02 & - & - & 3.28 & 3.07 \\
658A-31X-2, 70-74 & 271.19 & - & - & 3.13 & 3.11 \\
658A-31X-2, 102-104 & 271.52 & - & - & 3.06 & - \\
658A-31X-3, 63-67 & 272.52 & - & - & - & 3.15 \\
658A-31X-3, 72-74 & 272.62 & - & - & 3.25 & 3.26 \\
658A-31X-CC, 16-20 & 272.87 & - & - & 3.32 & - \\
658A-32X-1, 68-72 & 279.25 & - & - & 3.05 & 3.08 \\
658A-32X-1, 94-96 & 279.50 & - & - & 3.52 & 3.35 \\
658A-32X-CC, 18-22 & 279.72 & - & - & 3.12 & 3.01 \\
658A-33X-1, 70-74 & 288.77 & 2.40 & 0.41 & 3.17 & 3.11 \\
658A-33X-1, 102-104 & 289.08 & 2.04 & 0.01 & 3.14 & 3.11 \\
658A-33X-2, 70-74 & 290.27 & 2.24 & 0.24 & 3.05 & 2.91 \\
658A-33X-2, 102-104 & 290.53 & 2.39 & 0.12 & 2.94 & 3.00 \\
658A-33X-3, 70-74 & 291.52 & 2.43 & 0.01 & - & - \\
658A-33X-3, 102-104 & 291.83 & 2.58 & 0.10 & 3.34 & 3.37 \\
658A-33X-4, 12-16 & 292.13 & 2.41 & 0.48 & 3.02 & - \\
658A-33X-4, 70-74 & 292.70 & 2.36 & 0.19 & - & - \\
658A-33X-4, 102-104 & 293.01 & 2.03 & 0.09 & 2.93 & 3.06 \\
\hline & & & & & \\
\hline
\end{tabular}

tonic record. The range of interstage oxygen isotope variation is generally higher in the benthic record (up to $2.2 \%$ at the stage $5-6$ boundary) than in the planktonic record (up to $1.7 \%$, except for Termination I), an example of a high plankton to benthos difference in isotopic variation that is independent of high sedimentation rates. This indicates a generally stronger glacial-to-interglacial temperature overprint at $\sim 3000 \mathrm{~m}$ water depth than within the subtropical $\left(18^{\circ} \mathrm{N}\right)$ subsurface habitat of G. inflata (within and below the thermocline; Deuser et al., 1981).

\section{DISCUSSION}

\section{Stratigraphic Range of Hiatuses at Site 658}

At Site 658, the shipboard party (Ruddiman, Sarnthein, et al., 1988) identified a major hiatus covering approximately the time span between the Brunhes-Matuyama boundary and the last occurrence of $C$. macintyrei $(96.25-96.55 \mathrm{mbsf}, \mathrm{c.d}$.$) . In$ detail, however, the various stratigraphic methods assigned the hiatus to different core depths (Table 7). Both physical properties and nannofossil stratigraphy recorded a major event at about 96.4 mbsf (c.d.), whereas paleomagnetism indicated a break in the record only at or below $99 \mathrm{mbsf}$ (c.d.), about $2.5 \mathrm{~m}$ deeper.

Oxygen isotope stratigraphy at Site 658 (Fig. 4) enables us to make a clear identification of the numerical event taxonomy redefined by Prell et al. (1986) back to stage 16 . Within this stage, the planktonic record shows the slightly negative 16.3 isotopic event, which is not evident in the benthic record. The amplitude of the preceding short negative (warm) events at 95.08-96.16 mbsf (c.d.) is slightly lighter than that of the subsequent warm stages 15 and 13 and is typical of event 17.1. This identification is corroborated by a heavy $\delta^{13} \mathrm{C}$ value of $C$. wuellerstorfi (Table 3 and Fig. 4), which is characteristic of stage 17 in North Atlantic Deep Water (Sarnthein et al., 1984). Based on this assignment, the sedimentation rates between 
Table 4. Oxygen and carbon isotope data from uppermost $32 \mathrm{~m}$ of Site 659.

\begin{tabular}{|c|c|c|c|c|c|}
\hline $\begin{array}{l}\text { Core, section, } \\
\text { interval }(\mathrm{cm})\end{array}$ & $\begin{array}{l}\text { Depth } \\
\text { (c.d.- } \\
\text { mbsf) }\end{array}$ & \multicolumn{2}{|c|}{$\begin{array}{c}\text { C. } \\
\text { wuellerstorfi }\end{array}$} & \multicolumn{2}{|c|}{ G. inflata } \\
\hline $659 \mathrm{~A}-1 \mathrm{H}-1,12-16$ & 0.47 & 2.59 & 1.04 & 0.68 & 0.11 \\
\hline $659 \mathrm{C}-1 \mathrm{H}-1,92-94$ & 1.05 & 4.41 & 0.16 & 2.78 & 0.46 \\
\hline $659 \mathrm{~A}-1 \mathrm{H}-1,70-75$ & 1.06 & 4.18 & 0.11 & 1.93 & 0.10 \\
\hline $659 \mathrm{~A}-1 \mathrm{H}-1,102-105$ & 1.29 & 4.29 & 0.40 & 2.05 & 0.23 \\
\hline $659 \mathrm{C}-1 \mathrm{H}-1,140-142$ & 1.50 & 4.13 & 0.49 & 1.69 & 0.19 \\
\hline $659 \mathrm{~A}-1 \mathrm{H}-1,130-132$ & 1.56 & 3.66 & 0.61 & 1.47 & 0.17 \\
\hline $659 \mathrm{~A}-1 \mathrm{H}-2,12-16$ & 1.89 & 3.74 & 0.66 & 1.78 & 0.32 \\
\hline 659B-1H-2, 60-62 & 2.11 & 3.79 & 0.81 & 1.66 & 0.29 \\
\hline $659 \mathrm{C}-1 \mathrm{H}-2,62-64$ & 2.16 & 3.78 & 0.46 & 1.51 & 0.12 \\
\hline $659 \mathrm{C}-1 \mathrm{H}-2,92-94$ & 2.46 & 4.12 & 0.31 & 2.51 & 0.70 \\
\hline $659 \mathrm{~A}-1 \mathrm{H}-2,70-75$ & 2.51 & 3.71 & 0.03 & 2.01 & 0.39 \\
\hline $659 \mathrm{~A}-1 \mathrm{H}-2,102-105$ & 2.72 & 3.51 & 0.64 & 0.95 & 0.54 \\
\hline $659 \mathrm{C}-1 \mathrm{H}-2,140-142$ & 2.91 & 3.28 & 0.77 & 0.97 & 0.05 \\
\hline $659 \mathrm{~A}-1 \mathrm{H}-2,130-132$ & 2.99 & 3.61 & 0.59 & 1.22 & 0.36 \\
\hline $659 \mathrm{~A}-1 \mathrm{H}-3,12-16$ & 3.26 & 3.21 & 0.91 & 1.08 & 0.16 \\
\hline $659 \mathrm{~B}-1 \mathrm{H}-3,60-62$ & 3.61 & - & - & 1.58 & 0.65 \\
\hline $659 \mathrm{C}-1 \mathrm{H}-3,62-64$ & 3.63 & 3.23 & 0.56 & 1.51 & 0.45 \\
\hline $659 \mathrm{~A}-1 \mathrm{H}-3,70-75$ & 3.85 & 3.16 & 0.59 & 0.90 & 0.21 \\
\hline $659 \mathrm{C}-1 \mathrm{H}-3,92-84$ & 3.93 & 2.58 & 0.94 & 0.43 & 0.11 \\
\hline $659 \mathrm{~A}-1 \mathrm{H}-3,102-105$ & 4.16 & 2.15 & 0.68 & 0.41 & -0.42 \\
\hline $659 \mathrm{C}-1 \mathrm{H}-3,140-142$ & 4.41 & 4.35 & 0.27 & 1.70 & -0.40 \\
\hline $659 \mathrm{~A}-1 \mathrm{H}-3,130-132$ & 4.43 & 4.32 & 0.18 & 1.82 & -0.18 \\
\hline $659 \mathrm{~A}-1 \mathrm{H}-4,12-16$ & 4.73 & 4.16 & -0.18 & 2.06 & -0.12 \\
\hline $659 \mathrm{C}-1 \mathrm{H}-4,62-64$ & 5.13 & 4.04 & -0.07 & 2.10 & -0.19 \\
\hline $659 \mathrm{~B}-1 \mathrm{H}-4,60-62$ & 5.17 & 4.43 & 0.19 & 2.22 & 0.09 \\
\hline $659 \mathrm{~A}-1 \mathrm{H}-4,70-75$ & 5.26 & 4.14 & -0.06 & 1.28 & -0.51 \\
\hline $659 \mathrm{C}-1 \mathrm{H}-4,92-94$ & 5.43 & 4.13 & 0.25 & 1.99 & 0.21 \\
\hline $659 \mathrm{~A}-1 \mathrm{H}-4,102-104$ & 5.53 & 3.89 & 0.35 & 1.76 & -0.07 \\
\hline $659 \mathrm{~A}-1 \mathrm{H}-4,130-132$ & 5.81 & 3.86 & 0.01 & 1.80 & -0.17 \\
\hline $659 \mathrm{C}-1 \mathrm{H}-4,140-142$ & 5.91 & 3.80 & -0.01 & 1.78 & 0.09 \\
\hline $659 \mathrm{~A}-1 \mathrm{H}-5,12-16$ & 6.14 & 3.31 & 0.71 & 0.84 & -0.12 \\
\hline $659 \mathrm{C}-1 \mathrm{H}-5,62-64$ & 6.63 & 2.77 & 0.78 & 1.19 & 0.30 \\
\hline $659 \mathrm{~A}-1 \mathrm{H}-5,70-75$ & 6.73 & 2.92 & 0.83 & 1.04 & 0.08 \\
\hline $659 \mathrm{C}-1 \mathrm{H}-5,92-94$ & 6.93 & 3.56 & -0.32 & 2.38 & 0.11 \\
\hline 659 A-1H-5, 102-104 & 7.03 & 4.08 & 0.13 & 2.34 & 0.27 \\
\hline $659 \mathrm{~A}-1 \mathrm{H}-5,130-132$ & 7.31 & 3.76 & 0.34 & 1.88 & 0.25 \\
\hline $659 \mathrm{C}-1 \mathrm{H}-5,140-142$ & 7.41 & 2.99 & 0.29 & 1.28 & 0.29 \\
\hline $659 \mathrm{C}-1 \mathrm{H}-6,62-64$ & 8.13 & 3.77 & 0.24 & 1.73 & -0.21 \\
\hline $659 \mathrm{C}-1 \mathrm{H}-6,83-85$ & 8.34 & 3.98 & -0.06 & 1.81 & -0.09 \\
\hline $659 \mathrm{C}-1 \mathrm{H}-6,140-142$ & 8.91 & 3.46 & 0.46 & 1.63 & 0.44 \\
\hline $659 \mathrm{~A}-2 \mathrm{H}-1,18-22$ & 9.00 & 3.71 & 0.40 & 2.16 & 0.69 \\
\hline $659 \mathrm{C}-1 \mathrm{H}-7,23-25$ & 9.24 & 3.71 & 0.12 & 1.85 & 0.44 \\
\hline $659 \mathrm{C}-1 \mathrm{H}-7,35-37$ & 9.36 & 3.97 & 0.39 & 2.07 & 0.72 \\
\hline $659 \mathrm{~A}-2 \mathrm{H}-1,70-75$ & 9.63 & 3.40 & 0.29 & 1.48 & 0.31 \\
\hline $659 \mathrm{C}-2 \mathrm{H}-1,40-42$ & 9.90 & 3.54 & 0.17 & 1.99 & 0.09 \\
\hline $659 \mathrm{~A}-2 \mathrm{H}-1,100-102$ & 9.91 & 2.94 & 0.70 & 1.31 & 0.49 \\
\hline $659 \mathrm{~A}-2 \mathrm{H}-1,110-112$ & 10.01 & 2.65 & 0.29 & 0.88 & 0.27 \\
\hline $659 \mathrm{~A}-2 \mathrm{H}-1,130-132$ & 10.21 & 3.18 & -0.22 & 0.99 & 0.08 \\
\hline $659 \mathrm{C}-2 \mathrm{H}-1,92-94$ & 10.43 & 3.89 & -0.28 & 2.31 & -0.19 \\
\hline $659 \mathrm{~A}-2 \mathrm{H}-2,12-16$ & 10.54 & 4.21 & -0.21 & 2.22 & 0.04 \\
\hline $659 \mathrm{C}-2 \mathrm{H}-1,140-142$ & 10.91 & 3.85 & 0.08 & 1.82 & -0.02 \\
\hline $659 \mathrm{~A}-2 \mathrm{H}-2,70-75$ & 11.13 & 4.15 & -0.10 & 2.30 & 0.14 \\
\hline $659 \mathrm{C}-2 \mathrm{H}-2,40-42$ & 11.40 & 4.08 & -0.17 & 2.31 & 0.67 \\
\hline $659 \mathrm{~A}-2 \mathrm{H}-2,100-102$ & 11.41 & 3.97 & 0.11 & 2.37 & 0.52 \\
\hline $659 \mathrm{~A}-2 \mathrm{H}-2,130-132$ & 11.71 & 3.71 & 0.55 & 1.93 & 0.68 \\
\hline $659 \mathrm{C}-2 \mathrm{H}-2,92-94$ & 11.93 & 3.82 & 0.71 & 1.76 & 0.71 \\
\hline $659 \mathrm{~A}-2 \mathrm{H}-3,12-16$ & 12.04 & 3.29 & 0.43 & 1.34 & 0.25 \\
\hline $659 \mathrm{C}-2 \mathrm{H}-2,140-142$ & 12.41 & 3.21 & 0.37 & 1.45 & 0.57 \\
\hline $659 \mathrm{~A}-2 \mathrm{H}-3,70-75$ & 12.63 & 3.02 & 0.97 & 1.19 & 0.87 \\
\hline $659 \mathrm{C}-2 \mathrm{H}-3,40-42$ & 12.90 & 2.70 & 0.92 & 1.46 & 0.36 \\
\hline $659 \mathrm{~A}-2 \mathrm{H}-3,100-102$ & 12.91 & 2.70 & 0.74 & 1.50 & 0.59 \\
\hline $659 \mathrm{~A}-2 \mathrm{H}-3,130-132$ & 13.21 & 3.23 & 0.42 & 1.45 & -0.02 \\
\hline $659 \mathrm{C}-2 \mathrm{H}-3,92-94$ & 13.43 & 4.24 & -0.50 & 2.01 & -0.12 \\
\hline $659 \mathrm{~A}-2 \mathrm{H}-4,12-16$ & 13.54 & 3.92 & 0.04 & 2.13 & 0.10 \\
\hline $659 \mathrm{C}-2 \mathrm{H}-3,140-142$ & 13.91 & 4.41 & -0.39 & 1.96 & 0.01 \\
\hline $659 \mathrm{~A}-2 \mathrm{H}-4,70-75$ & 14.13 & 4.37 & 0.05 & 2.14 & 0.27 \\
\hline $659 \mathrm{C}-2 \mathrm{H}-4,40-42$ & 14.40 & 4.17 & 0.92 & 1.92 & 0.10 \\
\hline $659 \mathrm{~A}-2 \mathrm{H}-4,100-102$ & 14.41 & 4.13 & -0.24 & 2.48 & 0.24 \\
\hline $659 \mathrm{~A}-2 \mathrm{H}-4,130-132$ & 14.71 & 4.03 & 0.08 & 2.06 & 0.41 \\
\hline $659 \mathrm{C}-2 \mathrm{H}-4,92-94$ & 14.93 & 4.28 & 0.38 & 1.64 & 0.39 \\
\hline $659 \mathrm{~A}-2 \mathrm{H}-5,12-16$ & 15.05 & 3.77 & 0.49 & 2.22 & 0.67 \\
\hline $659 \mathrm{C}-2 \mathrm{H}-4,140-142$ & 15.41 & 3.31 & 0.51 & 0.96 & 0.31 \\
\hline $659 \mathrm{C}-2 \mathrm{H}-5,40-42$ & 15.91 & 3.31 & 1.10 & 1.47 & 0.92 \\
\hline $659 \mathrm{~A}-2 \mathrm{H}-5,80-82$ & 16.11 & 3.06 & 1.08 & 0.97 & 0.89 \\
\hline $659 \mathrm{C}-2 \mathrm{H}-5,92-94$ & 16.43 & 3.47 & 1.13 & 1.03 & 1.43 \\
\hline
\end{tabular}

Table 4 (continued).

\begin{tabular}{|c|c|c|c|c|c|}
\hline \multirow{2}{*}{$\begin{array}{l}\begin{array}{l}\text { Core, section, } \\
\text { interval }(\mathrm{cm})\end{array} \\
9 \mathrm{C}-2 \mathrm{H}-5,140-142\end{array}$} & \multirow{2}{*}{$\begin{array}{l}\begin{array}{l}\text { Depth } \\
\text { (c.d.- } \\
\text { mbsf) }\end{array} \\
16.91\end{array}$} & \multicolumn{2}{|c|}{$\begin{array}{c}C . \\
\text { wuellerstorfi } \\
\delta^{18} \mathrm{O} \quad \delta^{13} \mathrm{C}\end{array}$} & \multicolumn{2}{|c|}{$\delta^{18} \mathrm{O}$. inflata ${ }^{13} \mathrm{C}$} \\
\hline & & 3.54 & 0.97 & 0.93 & 0.62 \\
\hline $659 \mathrm{C}-2 \mathrm{H}-6,40-42$ & 17.41 & 3.55 & 0.78 & 1.51 & 0.34 \\
\hline $659 \mathrm{C}-2 \mathrm{H}-6,92-94$ & 17.93 & 4.18 & 0.38 & 2.05 & 0.69 \\
\hline $659 \mathrm{C}-2 \mathrm{H}-6,140-142$ & 18.41 & 3.28 & 0.42 & 1.22 & 0.29 \\
\hline $659 \mathrm{~A}-3 \mathrm{H}-1,70-75$ & 19.53 & 3.71 & 0.57 & 1.19 & 0.42 \\
\hline $659 \mathrm{~A}-3 \mathrm{H}-1,102-104$ & 19.83 & 3.06 & 0.74 & 1.42 & 0.44 \\
\hline $659 \mathrm{~A}-3 \mathrm{H}-1,130-132$ & 20.11 & 3.40 & 0.39 & 1.77 & -0.26 \\
\hline $659 \mathrm{~A}-3 \mathrm{H}-2,12-16$ & 20.44 & 4.42 & 0.01 & 2.41 & -0.08 \\
\hline $659 \mathrm{~A}-3 \mathrm{H}-2,70-75$ & 21.03 & 4.27 & -0.10 & 1.99 & -0.22 \\
\hline $659 \mathrm{~A}-3 \mathrm{H}-2,102-104$ & 21.33 & 3.84 & 0.15 & 2.25 & 0.14 \\
\hline $659 \mathrm{~A}-3 \mathrm{H}-2,130-132$ & 21.61 & 3.49 & 0.42 & 1.84 & 0.31 \\
\hline $659 \mathrm{~A}-3 \mathrm{H}-3,12-16$ & 21.94 & 2.98 & 0.82 & 1.17 & 0.34 \\
\hline $659 \mathrm{~A}-3 \mathrm{H}-3,70-75$ & 22.53 & 4.20 & 0.15 & 2.19 & 0.30 \\
\hline $659 \mathrm{~A}-3 \mathrm{H}-3,102-104$ & 22.83 & 3.31 & 0.70 & 1.33 & 0.46 \\
\hline $659 \mathrm{~A}-3 \mathrm{H}-3,130-132$ & 23.11 & 3.61 & 0.43 & 1.21 & -0.18 \\
\hline $659 \mathrm{~A}-3 \mathrm{H}-4,12-16$ & 23.44 & 4.11 & 0.08 & - & - \\
\hline $659 \mathrm{~A}-3 \mathrm{H}-4,70-75$ & 24.03 & 3.38 & 0.52 & 1.76 & 0.58 \\
\hline $659 \mathrm{~A}-3 \mathrm{H}-4,102-104$ & 24.33 & 2.63 & 0.26 & 1.61 & -0.02 \\
\hline $659 \mathrm{~A}-3 \mathrm{H}-4,130-132$ & 24.61 & 4.02 & -0.58 & 2.19 & -0.36 \\
\hline $659 \mathrm{~A}-3 \mathrm{H}-5,12-16$ & 24.94 & 3.86 & 0.09 & - & - \\
\hline $659 \mathrm{~A}-3 \mathrm{H}-5,70-75$ & 25.53 & 3.48 & 0.59 & 1.29 & 0.24 \\
\hline $659 \mathrm{~A}-3 \mathrm{H}-5,102-104$ & 25.83 & 3.03 & 0.12 & 1.71 & 0.42 \\
\hline $659 \mathrm{~A}-3 \mathrm{H}-5,130-132$ & 26.11 & 3.23 & 0.52 & 1.99 & 0.66 \\
\hline $659 \mathrm{~A}-3 \mathrm{H}-6,12-16$ & 26.44 & 3.71 & 0.01 & - & - \\
\hline $659 \mathrm{~A}-3 \mathrm{H}-6,74-79$ & 26.96 & 4.10 & -0.15 & 2.01 & -0.14 \\
\hline $659 \mathrm{~A}-3 \mathrm{H}-6,102-104$ & 27.23 & 3.64 & -0.07 & 2.32 & 0.06 \\
\hline $659 \mathrm{~A}-3 \mathrm{H}-6,130-132$ & 27.51 & 3.55 & 0.38 & 1.47 & -0.09 \\
\hline $659 \mathrm{~A}-4 \mathrm{H}-1,70-74$ & 29.02 & 3.59 & -0.15 & 1.61 & -0.17 \\
\hline $659 \mathrm{~A}-4 \mathrm{H}-1,102-104$ & 29.33 & 3.24 & 0.53 & 1.68 & 0.46 \\
\hline $659 \mathrm{~A}-4 \mathrm{H}-1,130-132$ & 29.61 & 3.34 & 0.22 & 1.51 & 0.17 \\
\hline $659 \mathrm{~A}-4 \mathrm{H}-2,12-16$ & 29.92 & 3.61 & 0.55 & - & - \\
\hline $659 \mathrm{~A}-4 \mathrm{H}-2,70-74$ & 30.50 & 3.77 & 0.59 & 1.56 & 0.58 \\
\hline $659 \mathrm{~A}-4 \mathrm{H}-2,102-104$ & 30.81 & 3.34 & 0.67 & 1.77 & 0.83 \\
\hline $659 \mathrm{~A}-4 \mathrm{H}-2,130-132$ & 31.09 & 3.25 & 0.56 & 1.20 & 0.52 \\
\hline $659 \mathrm{~A}-4 \mathrm{H}-3,12-16$ & 31.42 & 3.13 & 0.65 & - & - \\
\hline $659 \mathrm{~A}-4 \mathrm{H}-3,70-74$ & 32.00 & 3.72 & -0.10 & 1.88 & 0.45 \\
\hline $659 \mathrm{~A}-4 \mathrm{H}-3,102-104$ & 32.31 & 3.07 & 0.54 & 1.50 & 0.38 \\
\hline $659 \mathrm{~A}-4 \mathrm{H}-3,130-132$ & 32.59 & 2.61 & 0.75 & 0.92 & 0.33 \\
\hline $659 \mathrm{~A}-4 \mathrm{H}-4,12-16$ & 32.92 & 3.17 & 0.85 & - & - \\
\hline
\end{tabular}

events 16.2 and 17.1 (5.6 and $11.7 \mathrm{~cm} / \mathrm{k} . \mathrm{y}$.$) are somewhat$ lower than the average rates of the Brunhes Chron (Table 5).

The preceding cold stage below 96.4 mbsf (c.d.) is much less pronounced than the value expected for event 18.2 , especially at the high sedimentation rates in Site 658 (Prell et al., 1986; cf. Site 659, Fig. 5). Thus, we may ascribe this event either to stage 18.4 or to stage 58 in harmony with stages identified further downcore. Event 18.4 fits into the positive magnetic anomaly of the Brunhes Chron. On the other hand, possible evidence for stage 58 might derive from (1) the $\delta^{18} \mathrm{O}$ range of the event, which corresponds to that of the cold stages 60-70 preceding the hiatus; (2) the bipartite character of the stage in both the planktonic and benthic isotope record, which could be in harmony with the graphic record defined by Ruddiman et al. (1986); or (3) the nannofossil stratigraphy.

The preceding short-lasting but pronounced warm oxygen isotope stage at $97.99-98.83$ mbsf (c.d.) is equal or slightly more negative than the subsequent warm stages 17-13. By its amplitude it would match both stage 19 and stage 59. However, based on the Brunhes-Matuyama magnetic reversal lying close to the peak of this event (Fig. 4), the assignment to stage 19 is far more likely because the well-defined matching of the reversal and the pronounced warm stage (Shackleton and Opdyke, 1973, 1976) is hardly incidental.

Hence, we suggest that the main hiatus lies just below the Brunhes-Matuyama boundary, which is now regarded as real, 


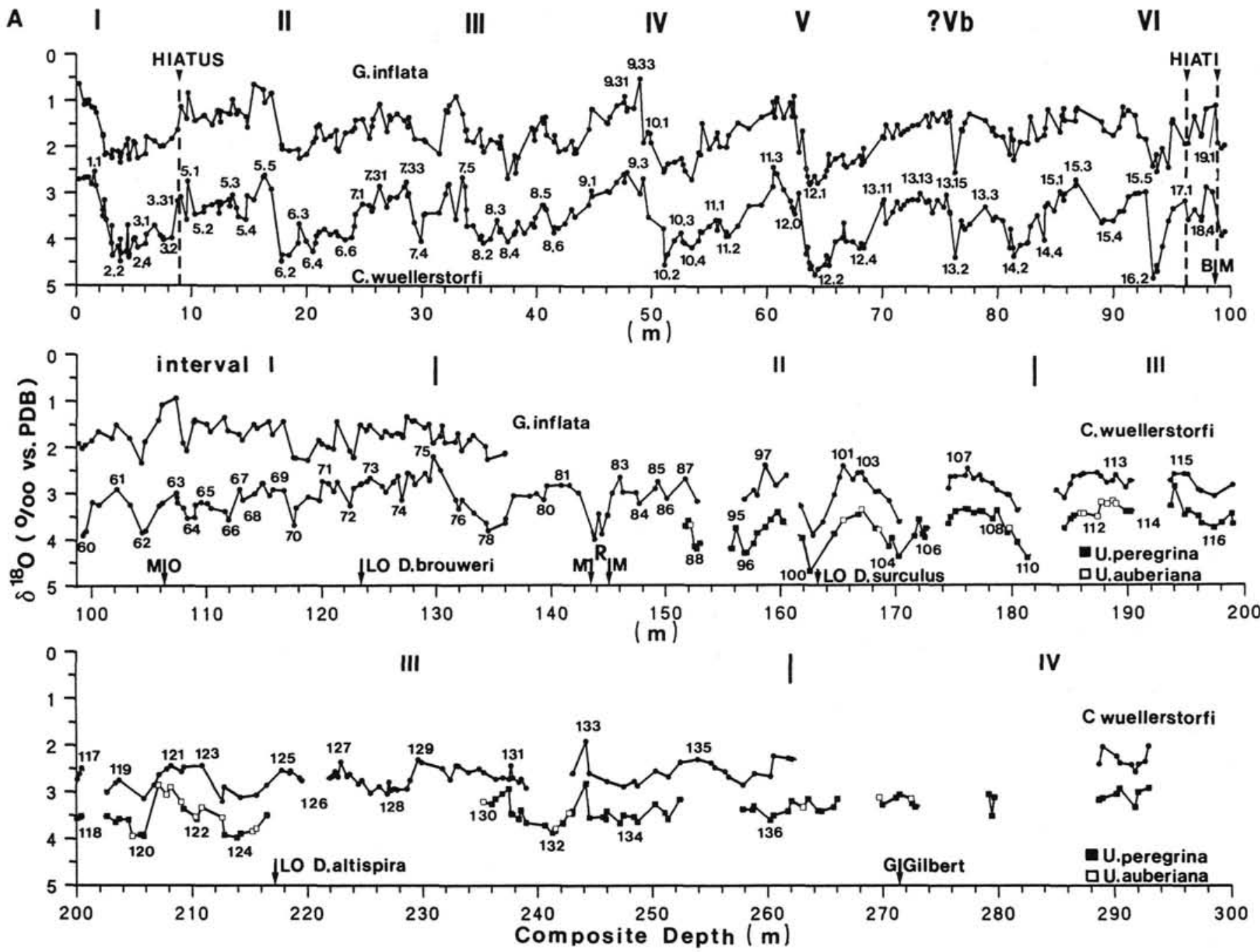

Figure 4. Summary of oxygen (A) and carbon (B) isotope data for Site 658 plotted vs. mbsf (c.d.). Proposed oxygen isotope midpoint stages, paleomagnetic boundaries, datums of nannofossil stratigraphy, hiatuses, and major intervals are also indicated. The isotope record of $G$. inflata ends at 135 mbsf because of the first occurrence of this species at $\sim 2 \mathrm{Ma}$. Roman numerals mark terminations.

in contrast to earlier suggestions in the Site 658 chapter (i.e., between the base of stage 19 and stage $60,0.735-1.575 \mathrm{Ma}$ ). The latter isotopic event clearly differs from stage 20 by its comparatively low amplitude and its morphological structure. The same holds true for the preceding stages 61 and 62 as compared with stages 21 and 22 . Consequently, the cool event subsequent to stage 19 is now confirmed as stage 18.4 instead of stage 58 .

Because this long hiatus near the Brunhes-Matuyama boundary clearly resulted from a major sediment slide (Ruddiman, Sarnthein, et al., 1988, pp. 105-219), it is not surprising to find a few reworked nannofossils from the underlying strata up to $2.5 \mathrm{~m}$ above the main hiatus. Furthermore, a slightly distorted stratigraphic sequence is evidenced by the delayed shift in the water content (Table 7) and by a second short hiatus spanning events $17.2-18.3$ at 96.4 mbsf (c.d.), a depth that corresponds to the top of the "main" hiatus as found in nannofossil stratigraphy. The second hiatus spans about 42 k.y.

A further hiatus is required at about 9-12 mbsf (c.d.) to explain the oxygen isotope records and the variation of sedimentation rates in the topmost $12 \mathrm{~m}$ at Site 658 (Fig. 4 and Table 5). The most recent stages 1,2 , and parts of 3 can be readily identified. The positive (cold) interval at $7.33-8.32$ mbsf (c.d.) must be assigned to event 3.2 and not to stage 4 because both the planktonic and benthic isotope records (Fig. 3B) lack the low $\delta^{13} \mathrm{C}$ values that are typical for isotope stage 4 (e.g., Shackleton, 1977; Sarnthein et al., 1984; Zahn et al., 1986). Accordingly, the preceding warm event at 8.83 mbsf (c.d.) should represent event 3.31 (Paterne et al., 1986) or 3.3 (Martinson et al., 1987). This event reaches a $\delta^{18} \mathrm{O}$ level as negative as the Alleröd stage (event 2.1), a range that appears characteristic only of records with very high time resolution like cores V19-30 (Shackleton et al., 1983), KET 8003, and KET 8004 (Paterne et al., 1986).

Further downcore, the narrow, extremely negative $\delta^{18} \mathrm{O}$ event at 9.69 mbsf (c.d.) observed in both the planktonic and benthic isotopic curves compares favorably with its $\delta^{18} \mathrm{O}$ value to stages 1 and 5.5. The peak may be best assigned to stage 5.1, although its low value exceeds all hitherto known levels, probably because of the extreme sedimentation rate given in our profile. Pflaumann (pers. comm.) found the last occurrence (LO) of Globorotalia hexagonus $60 \mathrm{~cm}$ on top of the event, at $9.1 \mathrm{mbsf}$ (c.d.), which marks the stage 5/4 boundary and hence confirms the stage 5.1 assignment of the event at 9.69 mbsf. It is also linked to an isolated, extreme 

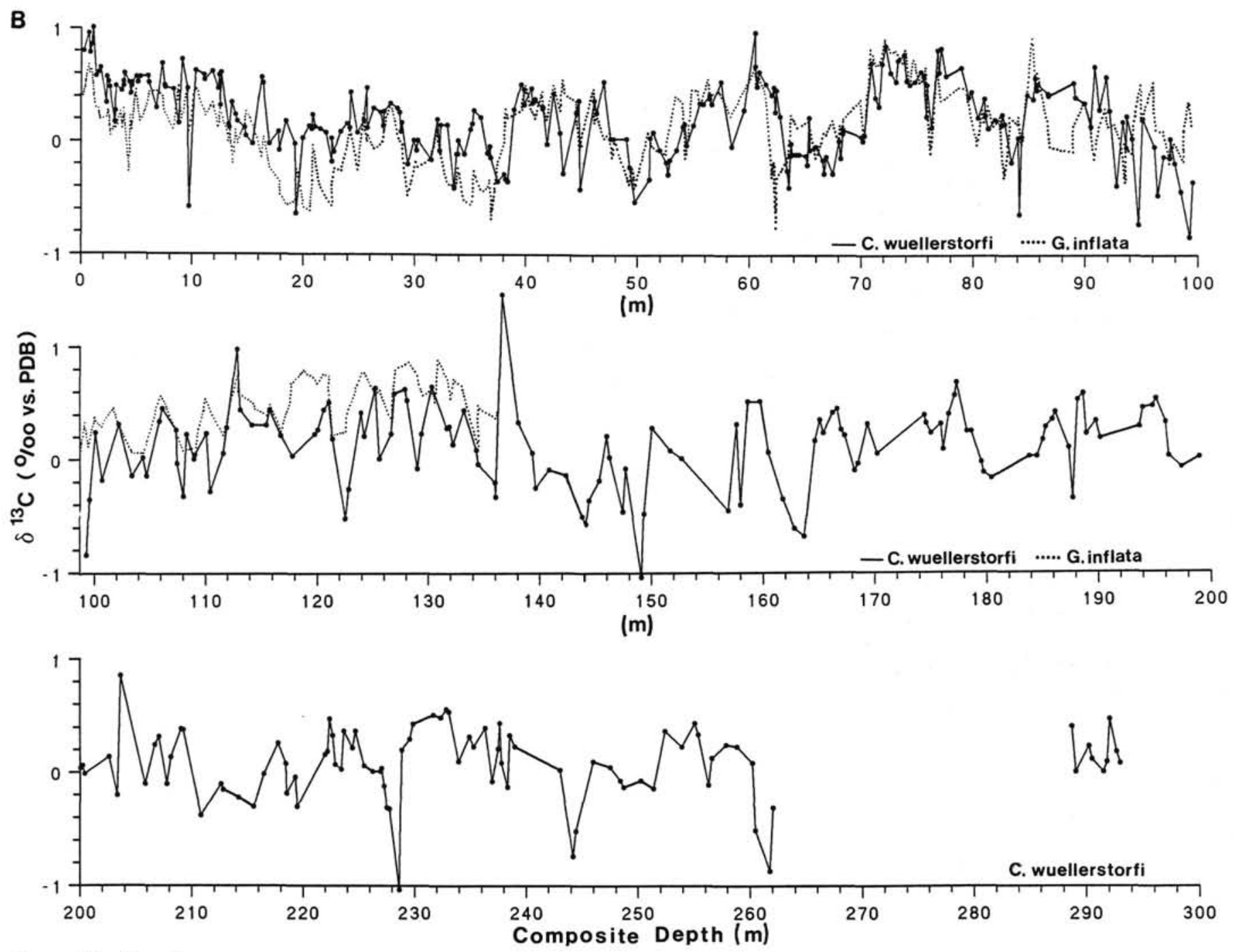

Figure 4 (continued).

$\delta^{13} \mathrm{C}$ minimum distinguished by a difference of about $1 \%$ in the benthic and $0.4 \%$ in the planktonic record (Fig. $4 \mathrm{~B}$ and Table 3).

In general, this minimum is characteristic of early stage 4 (Sarnthein et al., 1984), that is, it should actually be expected slightly further upcore. However, in summary, we may still conclude that the $\delta^{18} \mathrm{O}$ peak at 9.69 mbsf (c.d.) represents stage 5.1. The more positive event below, at 10.32 mbsf (c.d.), already marks the top part of stage 5.2. Therefore, stages 3.1 at 8.83 mbsf and 5.1 at 9.69 mbsf must be separated by a stratigraphic gap that spans stages 3.33 and 4 ; that is, from $\sim 50$ to 74.5 k.y., which is equivalent to $24,500 \mathrm{yr}$ or to a $3.5-\mathrm{m}$ sediment thickness (Table 5; sensu Paterne et al., 1986, and Martinson et al., 1987). Since the gap lies $1 \mathrm{~m}$ above the break between Cores 108-658B-2H and $-3 \mathrm{H}$ within an undisturbed sediment section, we assume that it is no artifact.

\section{High-Resolution Variability in the Oxygen Isotope Record during the Brunhes Chron (Site 658)}

In general, the planktonic and benthic isotope records of Figure 4A similarly depict a large number of highly resolved isotopic events during the last $730,000 \mathrm{yr}$. Based on the very high sedimentation rates averaging near $15 \mathrm{~cm} / \mathrm{k}$.y. and the largely undisturbed hemipelagic sediment record of Site 658 (Ruddiman, Sarnthein, et al., 1988), many in part new, smallscale features of these $\delta^{18} \mathrm{O}$ curves can now be substantiated and hardly be ascribed to products of bioturbation or to reworking. Other events, especially in the planktonic record, appear to be peculiarities of the local temperature history because the two isotopic curves disagree. Significant deviations between the two records only occur at Terminations I and III, where the onset of glacial-to-interglacial change in the benthic isotope record leads that of the planktonic one by about $4000 \mathrm{yr}$, a well-documented feature that we do not understand at this time (see further details below).

Figure 4 shows that the well-known sawtoothlike appearance of the $100-\mathrm{k} . \mathrm{y}$. climatic cycles is more pronounced during the last $600,000 \mathrm{yr}$ than in other single or stacked records (Prell et al., 1986). This is particularly apparent in the cycles of stages 6-7 and 13.2-15 and is most obvious in the benthic record. As pointed out by Shackleton and Hall (1983), the early cold events 12.2 and 16.2 appear more intensely "glacial" than the remaining even-numbered stages. Many of them have surprisingly constant isotopic values (e.g., $4.5 \%$ in the benthic record at events $2.2,6.2,10.2,13.2$, and 14.2; or about $4.0 \%$ at events $3.2,6.6,7.4,8.2,8.4,10.4,12.4$, and 14.4). As a consequence, there is no overall long-term trend in 
A
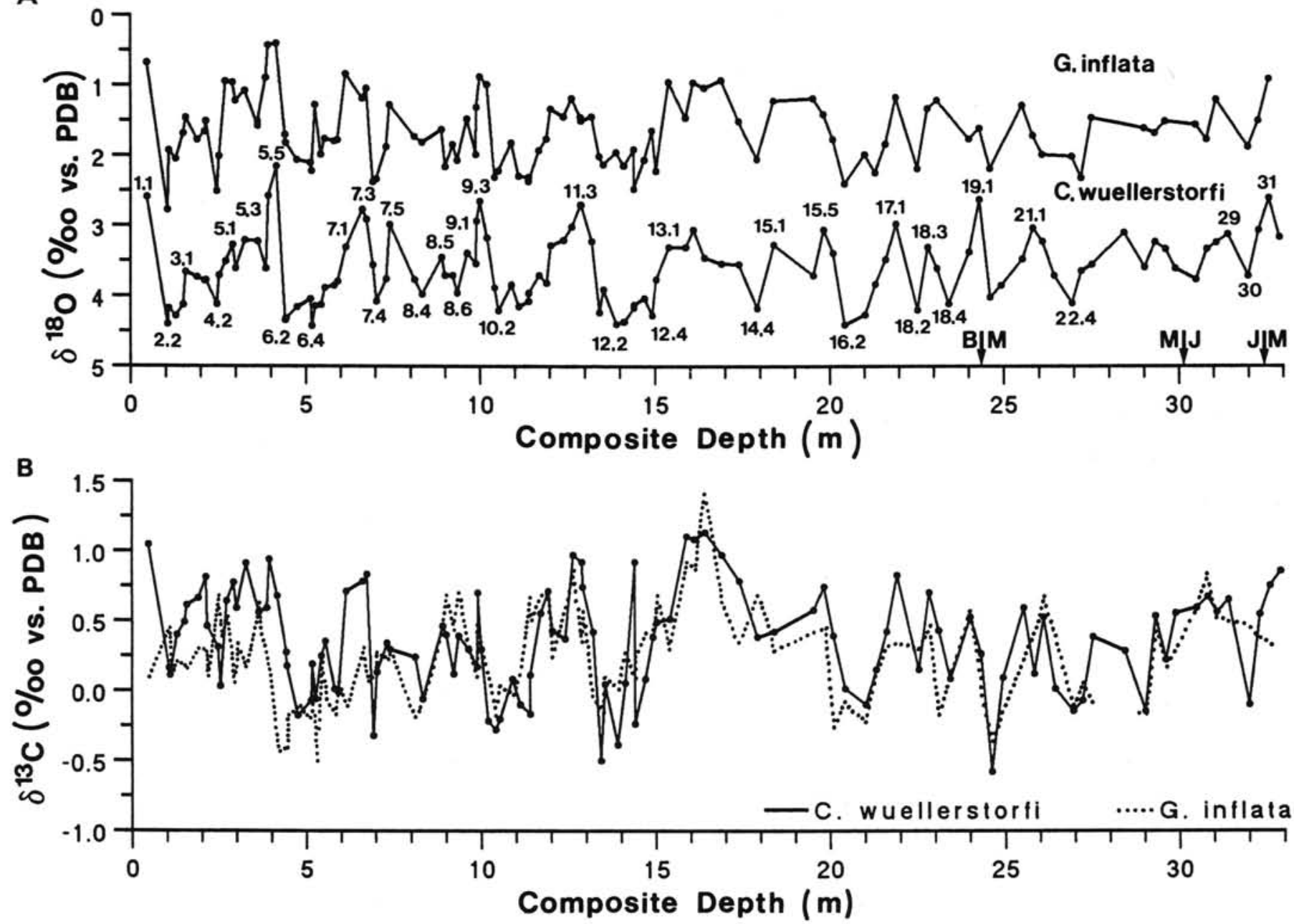

Figure 5. Summary of oxygen (A) and carbon (B) isotope data for Site 659 plotted vs. mbsf (c.d.). Proposed oxygen isotope midpoint stages, paleomagnetic boundaries, and datums of nannofossil stratigraphy are also indicated. Stages 24-31 after Ruddiman et al., 1986.

cooling or warming. Among the most negative events, events $11.3,9.3$, and 1.1 are slightly more "interglacial" in the benthic record than the other warm stages. In the planktonic record, event 9.33 is clearly more pronounced than the other stages, which may derive from local SST warming and, possibly, from a short-term local supply of fluvial water (Tiedemann et al., this vol.).

As a result of detailed time resolution, we identified the following new features in the benthic record and frequently in the planktonic record as well (Fig. 4). In contrast to existing stacked and single records (Prell et al., 1986; Sarnthein et al., 1984), events 5.1 and 7.5 now almost reach the "interglacial", range of the Holocene, with 7.5 appearing more "interglacial" than events 7.3 and 7.1. Furthermore, the by-now "medium cold" 'events 7.4, 14.2, and in particular 13.2, turn out to be as "glacial"' as most of the established glacial stages.

The single sample (108-658A-9H-6, 12-16 cm) that serves as the basis for the recognition of event 13.2 in the planktonic and benthic records has been obtained from a completely undisturbed core and sediment section (Ruddiman, Sarnthein, et al., 1988), where horizons with an equal isotopic composition occur only about $6 \mathrm{~m}$ further downcore or $12 \mathrm{~m}$ further upcore. Therefore, isotopic artifacts from sediment reworking are an unlikely explanation for the new magnitude of event 13.2. The extremely glacial character of event 13.2 is note- worthy, since it may add, in conjunction with the gradually deepening stage 14, another hitherto missed Termination "Vb" (Broecker and van Donk, 1970) to the long-term climatic record at about $515 \mathrm{k} . \mathrm{y}$.; that is, it may help to trace the 100,000 -yr cycle back to event 16.2 about $630,000 \mathrm{yr}$ ago.

Based on sedimentation rates between neighboring stage midpoints (Table 5), the outlined substages with newly discovered isotopic extremes did not last for more than about $4000-6000 \mathrm{yr}$. The same holds true for the isotopic peaks of a number of short-term warm events within glacial stages (e.g., events 6.3 and 12.3). This implies that a lot of the information on isotopic extremes must have been smoothed or lost in sediment records with only medium time resolution. In light of the recent results of Labeyrie et al. (1987), a large proportion of the short-term isotopic extremes may be linked to rapid temperature fluctuations in the North Atlantic Deep Water rather than to major waxing and waning of polar ice sheets.

The same might apply to a number of other well-established events in the oxygen isotope record of Site 658 (Fig. 4) that split off into "sub-substages." For example, event 7.3 split into 7.31 and $7.33,9.3$ into 9.31 and $9.33,10$ into 10.2 and 10.4 , and 13.1 into 13.11, 13.13, and 13.15. Most of these fine-scale events vary within a range of $0.5 \%$ and are substantiated by a match of the benthic and planktonic records and, moreover, by data points covering the flanks of the new 
Table 5. Names, midpoint ages, and sedimentation rates of $\delta^{18} \mathrm{O}$ stages and events at Site 658.

\begin{tabular}{cccc}
\hline $\begin{array}{c}\text { Depth } \\
\text { (c.d.- } \\
\text { mbsf) }\end{array}$ & $\begin{array}{c}\text { Age } \\
\text { (k.y.) }\end{array}$ & $\begin{array}{c}\text { Sedimentation } \\
\text { rate } \\
\text { (cm/k.y.) }\end{array}$ & Event \\
\hline 0.00 & 0.00 & & \\
1.63 & 8.00 & 20.4 & 1.1 \\
3.07 & 15.00 & 20.6 & Begin Termination 1 \\
6.13 & 27.00 & 25.5 & 3.1 \\
8.83 & 50.21 & 11.6 & 3.31
\end{tabular}

\section{H I A T U S}

$\begin{array}{rrrr}9.10 & 74.47 & & \text { Base Hiatus } \\ 9.69 & 79.25 & 12.3 & 5.1 \\ 11.13 & 90.95 & 12.3 & 5.2 \\ 14.80 & 110.79 & 18.5 & 5.4 \\ 16.38 & 123.82 & 12.1 & 5.5 \\ 18.16 & 135.10 & 15.8 & 6.2 \\ 19.38 & 142.28 & 17.0 & 6.3 \\ 20.58 & 152.58 & 11.7 & 6.4 \\ 23.40 & 183.30 & 9.2 & 6.6 \\ 24.90 & 193.07 & 15.4 & 7.1 \\ 25.71 & 200.57 & 10.8 & 7.2 \\ 27.56 & 215.54 & 12.4 & 7.3 \\ 29.99 & 224.89 & 26.0 & 7.4 \\ 33.58 & 240.19 & 23.5 & 7.5 \\ 35.38 & 249.00 & 20.4 & 8.2 \\ 36.58 & 257.00 & 15.0 & 8.3 \\ 37.49 & 265.67 & 10.5 & 8.4 \\ 40.51 & 288.54 & 13.2 & 8.5 \\ 44.77 & 310.00 & 19.9 & 9.1 \\ 48.75 & 331.00 & 19.0 & 9.3 \\ 51.12 & 341.00 & 23.7 & 10.2 \\ 55.73 & 368.00 & 17.1 & 11.1 \\ 56.55 & 375.00 & 11.7 & 11.2 \\ 60.58 & 405.00 & 13.4 & 11.3 \\ 62.43 & 423.00 & 10.3 & 12.0 \\ 62.80 & 426.00 & 12.3 & 12.1 \\ 64.19 & 434.00 & 17.4 & 12.2 \\ 68.25 & 471.00 & 11.5 & 12.4 \\ 70.18 & 481.00 & 17.5 & 13.11 \\ 76.32 & 513.00 & 19.2 & 13.2 \\ 81.43 & 538.00 & 20.4 & 14.2 \\ 84.07 & 563.00 & 10.6 & 14.4 \\ 85.49 & 574.00 & 12.9 & 15.1 \\ 90.04 & 607.00 & 13.8 & 16.4 \\ 93.37 & 631.00 & 13.9 & 17.1 \\ 96.16 & 668.00 & 7.5 & \end{array}$

\section{H I A T U S}

$\begin{array}{ll}96.41 & 710.00 \\ 97.55 & 721.00 \\ 98.75 & 730.00 \\ 98.83 & 731.00\end{array}$

$\begin{array}{rc} & \text { Base Hiatus } \\ 10.9 & 18.4 \\ 10.9 & \text { Brunhes/Matuyama } \\ 8.0 & 19.1\end{array}$

H I A T U S

$\begin{array}{rr}99.30 & \mathrm{a}_{1575.00} \\ 102.24 & \mathrm{a}_{1595.00} \\ 104.43 & \mathrm{a}_{1616.00} \\ 106.65 & 1631.00 \\ 107.43 & \mathrm{a}_{1636.00} \\ 123.55 & \mathrm{~b}_{1830.00} \\ 134.51 & \mathrm{~b}_{1941.00} \\ 143.54 & 2024.00 \\ 143.83 & \mathrm{~b}_{2027.00} \\ 145.01 & 2044.00 \\ 152.75 & \mathrm{~b}_{2152.00} \\ 155.03 & 2184.00\end{array}$

Table 5 (continued).

\begin{tabular}{lccl}
\hline $\begin{array}{c}\text { Depth } \\
\text { (c.d.- } \\
\text { mbsf) }\end{array}$ & $\begin{array}{c}\text { Age } \\
\text { (k.y.) }\end{array}$ & $\begin{array}{c}\text { Sedimentation } \\
\text { rate } \\
\text { (cm/k.y.) }\end{array}$ & \multicolumn{1}{c}{ Event } \\
\hline 205.34 & 2828.00 & 9.6 & Stage 120 \\
207.63 & 2851.00 & 9.6 & Stage 121 \\
210.44 & 2880.00 & 9.6 & Stage 122 \\
211.77 & 2894.00 & 9.6 & Stage 123 \\
213.95 & 2917.00 & 9.6 & Stage 124 \\
217.15 & 2950.00 & 9.6 & LO D. altispira \\
217.81 & 2956.00 & 12.1 & Stage 125 \\
220.78 & 2980.00 & 12.1 & Stage 126) \\
222.93 & 2978.00 & 12.1 & Stage 127 \\
226.97 & 3031.00 & 12.1 & Stage 128 \\
229.63 & 3053.00 & 12.1 & Stage 129 \\
236.07 & 3107.00 & 12.1 & Stage 130 \\
237.53 & 3119.00 & 12.1 & Stage 131 \\
241.28 & 3150.00 & 12.1 & Stage 132 \\
244.22 & 3174.00 & 12.1 & Stage 133 \\
247.50 & 3202.00 & 12.1 & Stage 134 \\
253.92 & 3255.00 & 12.1 & Stage 135 \\
257.88 & 3288.00 & 12.1 & Stage 136 \\
271.45 & 3400.00 & 12.1 & Gauss/Gilbert \\
\hline
\end{tabular}

Note: Magnetic and nannofossil ages are according to Ruddiman, Sarnthein, et al., 1988, modified by Chepstow-Lusty et al., this vol., and Raymo et al., 1988. Ages of stages 1-19 are based on Imbrie et al., 1984, Martinson et al., 1987, and Paterne et al., 1986. Stages $117-137$ are newly defined in this paper, c.d. = composite depth; LO = last occurrence.

a Stages and ages are based on Ruddiman et al., 1986.

${ }^{b}$ Stages and ages are based on Raymo et al., 1988.

(sub-)events. A similar systematic splitting of the oxygen isotopic events was first recognized by Paterne et al. (1986) and Martinson et al. (1987) for stages 2-6.

A most interesting detail observed in the high-frequency range of the curves from Site 658 is the multiple repetition of cold events comparable with the Younger-Dryas. They occur, in addition to the event at Termination I, with Terminations III, IV, V, and VI? in the planktonic record and with Terminations IV and V in the benthic one. The short-term negative $\delta^{18} \mathrm{O}$ reversals (comparable with the Alleröd) lie about $0.65-$ $1.30 \mathrm{~m}$ downcore from the $\delta^{18} \mathrm{O}$ minima marking the subsequent peak interglacial stages. Likewise, the positive reversals (comparable with Younger-Dryas) lie about $60-65 \mathrm{~cm}$ upcore from the preceding cold stages.

Although bioturbational downcore mixing across this distance cannot be fully excluded, it appears unlikely. This assumption is corroborated by the frequency distribution of $G$. inflata specimens, which culminates across the terminations, ranging from 2 to 13 specimens $/ \mathrm{cm}^{3}$ at Termination IV and from 1 to $21 / \mathrm{cm}^{3}$ at Termination V. Moreover, in the case of Termination $\mathrm{V}$, the "warm" isotopic excursion is marked by three data points (not resolved in the small-scale Fig. 4) from a 19-cm-thick section. In contrast to $G$. inflata, $C$. wuellerstorfi shows a markeddowncoredecreaseinfrequency(from 4.5 to 0 -1 specimens/ $\mathrm{cm}^{3}$ ) below the short-term positive $\delta^{18} \mathrm{O}$ excursion within Terminations IV and V. Hence, the preceding short "warm" excursion also may be a result of bioturbational downcore mixing. However, the sum of our evidence suggests that the $\delta^{18} \mathrm{O}$ excursions correlating with the terminations are real.

Thus, we may infer that the presently much discussed climatic reversal during the last deglaciation (e.g., Labeyrie and Berger, 1987) presents a phenomenon that is linked more generally to the major climatic amelioration phases during the Brunhes Chron. Apparently, these reversals are more closely associated with SST variations than with changes of ice volume and deep-water temperatures, because they occur 
Table 6. Sedimentation rates for the uppermost $32.5 \mathrm{~m}$ of Site 659 based on $\delta^{18} \mathrm{O}$ stratigraphy.

\begin{tabular}{|c|c|c|c|}
\hline $\begin{array}{l}\text { Depth } \\
\text { (c.d.- } \\
\text { in m) }\end{array}$ & $\begin{array}{c}\text { Age } \\
(\mathrm{k} . \mathrm{y} .)\end{array}$ & $\begin{array}{c}\text { Sedimentation } \\
\text { rate } \\
(\mathrm{cm} / \mathrm{k} . \mathrm{y} .)\end{array}$ & Event \\
\hline 0.47 & 8.00 & 5.9 & 1.1 \\
\hline 1.53 & 27.00 & 5.6 & 3.1 \\
\hline 2.46 & 65.00 & 2.5 & 4.2 \\
\hline 2.82 & 79.25 & 2.5 & 5.1 \\
\hline 4.16 & 123.82 & 3.0 & 5.5 \\
\hline 4.43 & 135.10 & 2.4 & 6.2 \\
\hline 5.17 & 152.58 & 4.2 & 6.4 \\
\hline 6.14 & 193.07 & 2.4 & 7.1 \\
\hline 6.73 & 215.54 & 2.6 & 7.3 \\
\hline 6.93 & 224.89 & 2.1 & 7.4 \\
\hline 7.41 & 240.19 & 3.1 & 7.5 \\
\hline 9.36 & 299.00 & 3.3 & 8.6 \\
\hline 9.63 & 311.00 & 2.3 & 9.1 \\
\hline 10.01 & 331.00 & 1.9 & 9.3 \\
\hline 10.54 & 341.00 & 5.3 & 10.2 \\
\hline 12.90 & 405.00 & 3.7 & 11.3 \\
\hline 13.91 & 434.00 & 3.5 & 12.2 \\
\hline 14.93 & 471.00 & 2.8 & 12.4 \\
\hline 15.41 & 492.00 & 2.3 & 13.1 \\
\hline 17.93 & 563.00 & 3.6 & 14.4 \\
\hline 19.83 & 617.00 & 3.5 & 15.5 \\
\hline 20.44 & 633.00 & 3.8 & 16.2 \\
\hline 21.94 & 668.00 & 4.3 & 17.1 \\
\hline 22.53 & 695.00 & 2.2 & 18.2 \\
\hline 24.30 & 730.00 & 5.1 & Brunhes/Matuyama \\
\hline 24.33 & 731.00 & 3.0 & 19.1 \\
\hline 25.53 & 774.00 & 2.8 & 21.1 \\
\hline 26.96 & 814.00 & 3.6 & 22.4 \\
\hline 30.10 & 910.00 & 3.3 & Matuyama/Jaramillo \\
\hline 32.50 & 980.00 & 3.4 & Jaramillo/Matuyama \\
\hline
\end{tabular}

Note: Ages are based on Imbrie et al., 1984; Martinson et al., 1987; Ruddiman et al., 1986; and Ruddiman, Sarnthein, et al., 1988, pp. 221-325. c.d. = composite depth.

more regularly in the planktonic than in the benthic record, which has suffered, however, from low specimen abundance.

\section{The Early Pleistocene and Pliocene Record at Site 658}

Based on the amplitude of its oscillations, the Site $658 \delta^{18} \mathrm{O}$ record (Fig. 4) indicates four major intervals of climatic variability during the early Pleistocene and late Pliocene; that is, between the major hiatus at $99 \mathrm{~m}$ and $295 \mathrm{mbsf}$ (c.d.), at 1.575 and $3.6 \mathrm{Ma}$ (interpolated ages after Table 5). To some degree, the boundaries between these intervals parallel events suggested by Keigwin (1986). The uppermost interval at 99-130 mbsf (c.d.), as shown in Figure 4A, depicts a gradual downcore decrease in climatic and isotopic variability from about $1 \%$ near 118 mbsf $(1.77 \mathrm{Ma})$ to less than $0.5 \%$ near 130 mbsf (c.d.), about $1.90 \mathrm{Ma}$. The second interval extends down to $182 \mathrm{mbsf}$ (c.d.), equivalent to about $2.6 \mathrm{Ma}$ and, in comparison with other published records (Fig. 6), down to 194 mbsf (c.d.) or $2.71 \mathrm{Ma}$. Interval II comprises at least eight marked cycles with relative $\delta^{18} \mathrm{O}$ fluctuations of up to $1.6 \%$ and a number of shorter and/or smaller cycles that are superimposed. The third interval continues down to $262 \mathrm{mbsf}$ (c.d.), corresponding to $3.32 \mathrm{Ma}$, and consists of seven major cycles with benthic $\delta^{18} \mathrm{O}$ fluctuations reaching up to $1.0 \%$. The $\delta^{18} \mathrm{O}$ cycles of interval IV, below 262 mbsf (c.d.) in Figure 4A, are documented incompletely but are apparently smaller than $0.5-0.6 \%$.

This large-scale subdivision scheme matches in great detail (Fig. 6) the oxygen isotope records of Sites 552 (Shackleton et al., 1984., suppl. by Curry and Miller, this vol.), 606 (Keigwin, 1986), and 665 (Curry and Miller, this vol.). Individual isotopic oscillations are equally similar. Figure 6 shows that the $\delta^{18} \mathrm{O}$ record of Site 658 is characterized by the smoothest alignment of data points with major oscillations and the lowest number of subordinate "one-point" fluctuations of the four records compared prior to $2.4 \mathrm{Ma}$. This feature is possibly linked to the high sedimentation rates at Site 658 . Note that none of the major glacial cycles between 1.9 and $2.6 \mathrm{Ma}$ has developed a sawtooth shape ending with an abrupt termination like the $100-\mathrm{k} . \mathrm{y}$. cycles of the Brunhes Chron, possibly implying a different mechanism of deglaciation.

The early Quaternary to late Pliocene isotopic oscillation record of intervals I and II at Site 658 compares generally well with the orbitally tuned stage sequence $60-116$ defined by Ruddiman et al. (1986) and Raymo et al. (1988) at Site 607. However, at well-documented extreme isotopic events (such as stage 100), the amplitudes at Site 658 clearly exceed those of Site 607 by up to one-third. Based on the tuned time scale of Raymo et al. (1988), the Réunion I magnetic event, which matches stage 82 at Site 658 , has a tuned age of 2.024-2.044 m.y., as compared with an age of 2.01-2.04 m.y. according to Mankinen and Dalrymple (1979).

Apart from the artificial gap outlined at the core break about 155 mbsf (c.d.), however, a number of further oxygen isotopic stages that were defined at Site 607 can hardly be identified at Site 658 below $155 \mathrm{mbsf}$, that is, prior to $2.3 \mathrm{Ma}$. "Glacial" stage 98 remained unidentified at Site 658 because of a lack of benthic foraminifer specimens in our samples within a section that was $\sim 1.40$ m thick, a lack common to many Pliocene cold stages at Site 658 . However, stage 98 is well documented as a marked but short event $(<15,000 \mathrm{yr})$ at Sites 552, 606, 607, and 665. Stage 100, the largest isotopic excursion of the late Pliocene, marks a slight change in sedimentation rates at Site 658 from about $96 \mathrm{~m} / \mathrm{m}$.y. below to

Table 7. Position of hiatus near Brunhes-Matuyama Boundary (BMB) at Site 658 determined by different stratigraphic methods.

\begin{tabular}{|c|c|c|c|c|}
\hline $\begin{array}{l}\text { Stratigraphic } \\
\text { method }\end{array}$ & $\begin{array}{l}\text { Observed } \\
\text { features }\end{array}$ & $\begin{array}{l}\text { Depth } \\
\text { (c.d.-mbsf) }\end{array}$ & $\begin{array}{c}\text { Hole } 658 \mathrm{~A} \\
\text { (original depth } \\
\text {-mbsf) }\end{array}$ & $\begin{array}{c}\text { Age } \\
\text { range } \\
(\mathrm{Ma})\end{array}$ \\
\hline${ }^{\mathrm{a}}$ Nannofossils & $\begin{array}{l}\text { LO C. macintyrei } \\
\text { LO } H \text {. sellii along with BMB }\end{array}$ & $96.25-96.65$ & $99.1-99.4$ & $\begin{array}{l}>1.450 \\
\text { (base of hiatus) }\end{array}$ \\
\hline${ }^{\text {aphysical properties }}$ & Shift in average water content by about $12 \%$ & 96.4 & 99.25 & - \\
\hline a Paleomagnetism & $\begin{array}{l}\text { Magnetic reversal near expected base of Brunhes Chron } \\
\text { along with older nannofossil datum }\end{array}$ & 98.75 & 101.60 & $\begin{array}{l}<0.730 \text { (top of } \\
\text { hiatus) }\end{array}$ \\
\hline$\delta^{18} \mathrm{O}$ record & $\begin{array}{l}{ }^{b} \text { Break in } \delta^{18} \mathrm{O} \text { curves by } 0.95(0.8) \% \text { between base of } \\
\text { Stages } 19 \text { and } 60\end{array}$ & 98.83-98.98 & $101.68-101.83$ & $0.731-1.571$ \\
\hline
\end{tabular}

a Data from Ruddiman, Sarnthein, et al., 1988, pp. 105-219.

$\mathrm{b}$ The identification of stage 19 is based on coincidence with the BMB; the identification of stage 60 is based on graphic correlation of isotopic event series 99-109 mbsf (c.d.) to stages 60-64, the oldest of which is linked to the top Olduvai reversal (Ruddiman et al., 1986). 

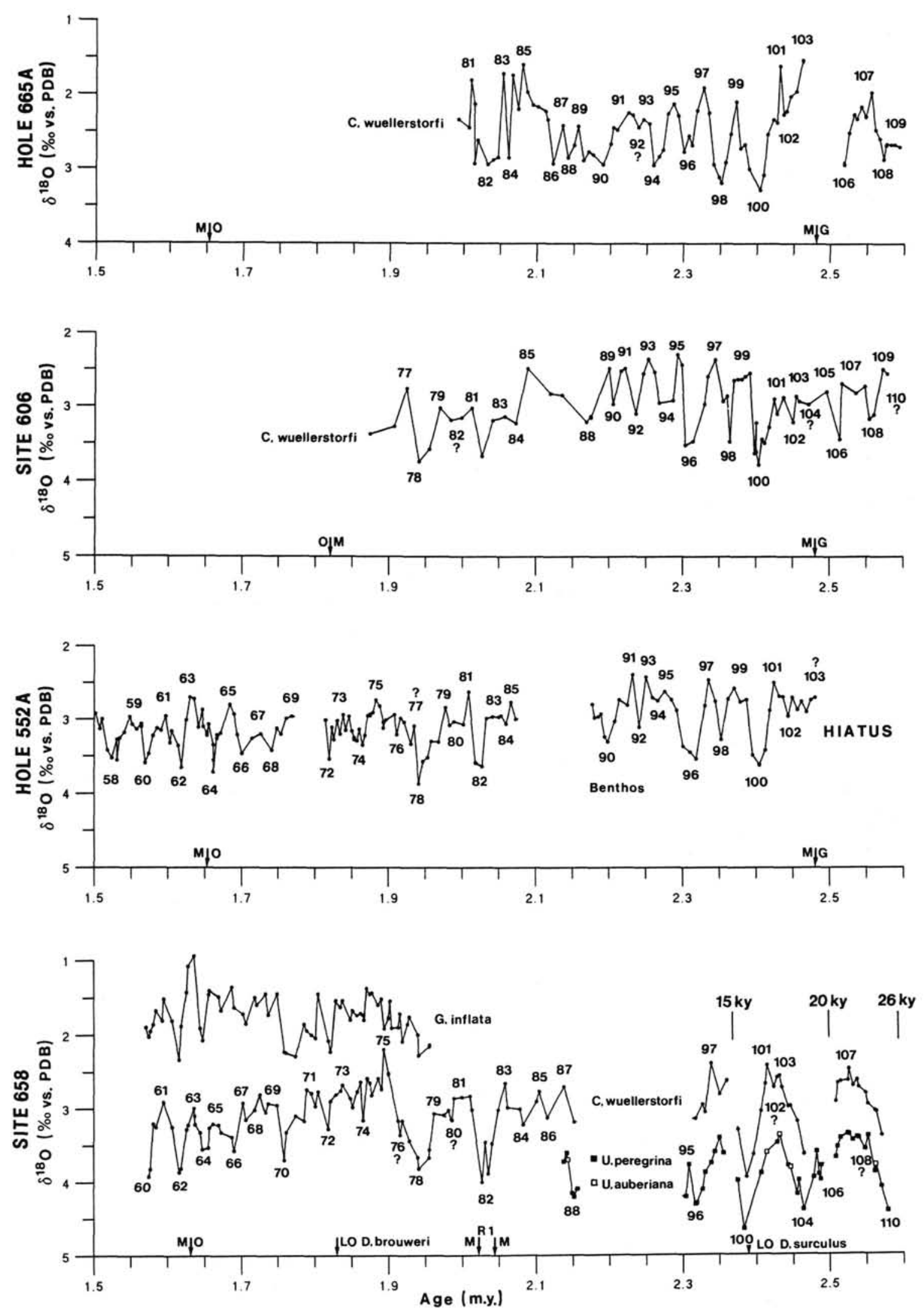

Figure 6. Oxygen isotope data of Site 658 from 100 to $296 \mathrm{mbsf}$ (c.d.) plotted on a time scale of linear adjustment between orbitally tuned ages of $\delta^{18} \mathrm{O}$ stages 60-100 (Raymo et al., 1988), magnetic reversals, and age-calibrated nannofossil datums (Table 5). The duration of major gaps in the $\delta^{18} \mathrm{O}$ record are indicated in 1000-yr (k.y.) increments. Oxygen isotope records for Hole 552A (Shackleton and Hall 1984, suppl. by Curry and Miller, this vol.), Site 606 (Keigwin, 1986), and Hole 665A (Curry and Miller, this vol.) are plotted for comparison. The duration of the major hiatus at Hole 552A (stages 104-112) is redefined according to $\delta^{18} \mathrm{O}$ stratigraphy. 

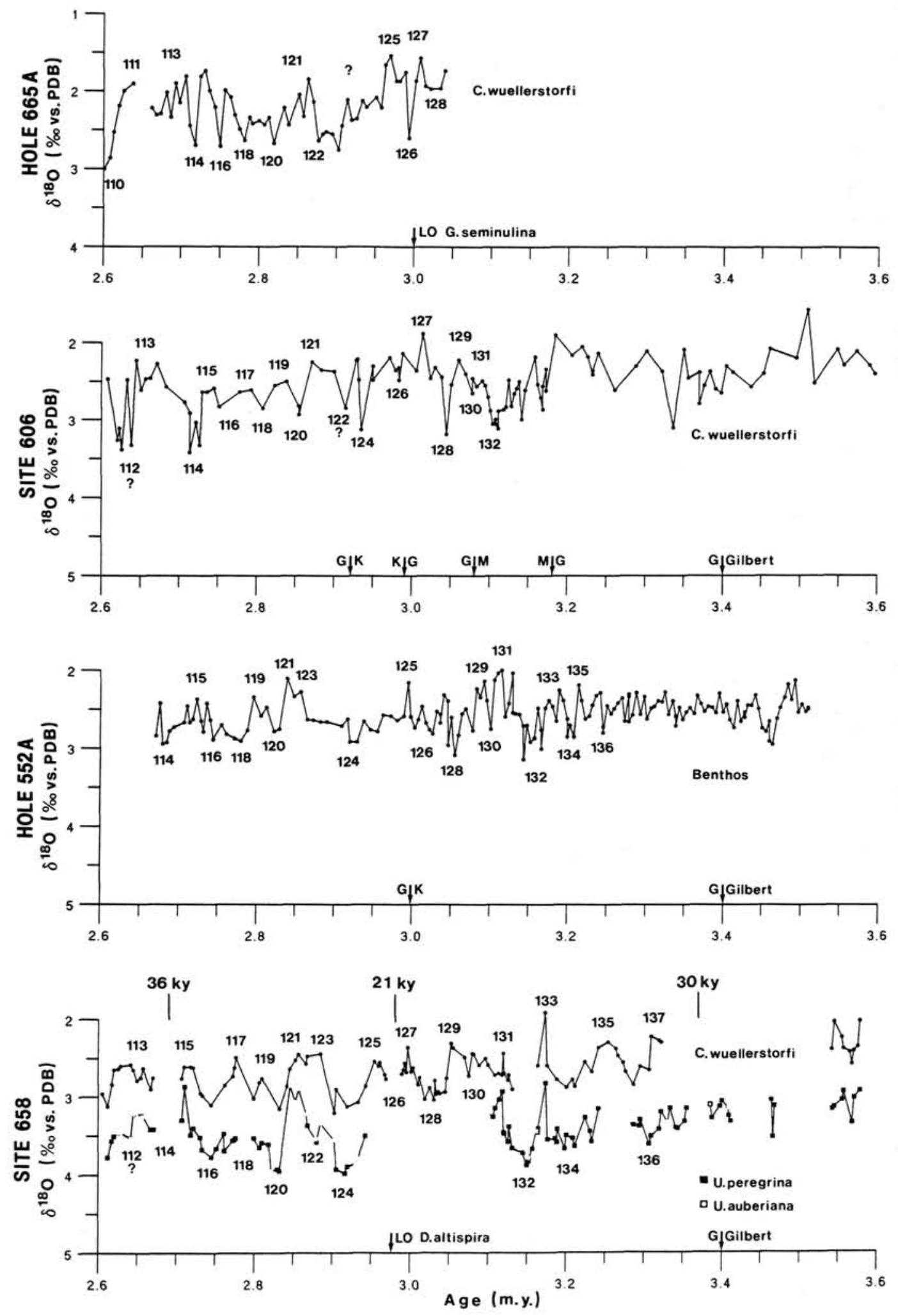

Figure 6 (continued). 
$86 \mathrm{~m} / \mathrm{m} . \mathrm{y}$. and less, above the extinction of $D$. surculus, a change that is probably related to long-term variations in land climate and the regime of terrigenous sediment supply (Tiedemann et al., this vol.).

Further below, cold stages 102, 108, and 112 cannot be clearly identified despite sufficient sampling resolution and the absence of core breaks in the particular depth ranges of the Site 658 record. Stage 102 is very insignificant also at Site 607 (Raymo et al., 1988) and, hence, may be negligible. Stage 108 may be contained in the flank between stages 107 and 110 . Stage 112 can hardly be detected within the "warm" oscillation of stages $111 / 113$, which are well documented by both $C$. wuellerstorfi and Uvigerina sp. data.

The $\delta^{18} \mathrm{O}$ stage 110 marks the first major glacial event at Site 658 , that is, a major climatic deterioration at about 2.58 Ma. The preceding major cold stage 114 was almost lost by a (core break and) data gap of 36 k.y. at Site 658. In contrast, the records from Sites 606 and 665 show that stage 114 has been as pronounced as stage 110 . Hence, the first major glaciation occurred at about $2.63-2.72 \mathrm{Ma}$. This datum predates the first major glacial isotopic event originally defined at stage 100 of Site 552 (Shackleton et al., 1984) by almost $300,000 \mathrm{yr}$. Based on this discrepancy and on the fact that several isotopic oscillations are missed in the Site 552 record between the extinction datums of $D$. surculus and $D$. tamalis, we surmise that this sediment section from Site 552 was lost by a hitherto unrecognized hiatus near the Matuyama-Gauss boundary comprising stages 104-112 (Fig. 6). Curry and Miller (this vol.) arrived at a similar conclusion, but they located the gap about $1 \mathrm{~m}$ further downcore at a core break, an approach that strongly impedes the recognition of isotopic stages back to about stage 116 .

Based on the close affinities between the records from different sites (Fig. 6) and the average time resolution of 1 sample every $5240 \mathrm{yr}$ in the Site 658 record prior to $2.3 \mathrm{Ma}$, we propose to continue the $\delta^{18} \mathrm{O}$ stage classification of Raymo et al. (1988) by naming and dating the $\delta^{18} \mathrm{O}$ stages of the early late Pliocene back to 3.3 Ma. Designations for stages 116 through 137 are shown in Figures 4 and 6 and listed in Table 5.

The 21 proposed glacial and interglacial stages start below glacial stage 116, which is the last of the less pronounced glacial events characteristic of large-scale interval III. The 7 major climatic cycles of stages 116, 120, 124, 128, 132, 134, and 136 continue to indicate $40-$ and $100-\mathrm{k} . \mathrm{y}$. periodicities, because many warm stages are subdivided by minor climatic oscillations (stages 118, 122, 126, 128, and 130).

The extremely negative level of stage 133 in both the $C$. wuellerstorfi and the Uvigerina sp. records of Site 658 marks the onset of a major long-term increase in the oxygen isotopic range of both warm and cold stages. Prior to stage 115 in the Uvigerina sp. record and prior to stages $127 / 129$ in the $C$. wuellerstorfi record, the "interglacial" values were lowered by up to $0.5 \%$ when compared with the peak Holocene level, particularly conspicuous also at Sites 552 and 606 (Fig. 6). This superimposed isotopic shift at $3.05-2.7 \mathrm{Ma}$, which is also reflected by the "glacial" values, was first depicted by Shackleton and Opdyke (1976) and discussed extensively by Prell (1984) and Keigwin (1986).

Based on planktonic-benthic foraminifer differences in the $\delta^{18} \mathrm{O}$ shift at Site 606 , Keigwin (1986) related about $0.5 \%$ of the general $\delta^{18} \mathrm{O}$ increase at stage 132 to a major deep-water cooling by more than $2^{\circ} \mathrm{C}$. Another $0.5 \%$, which was recorded in the planktonic and benthic foraminifer records, was ascribed to an early phase of small but long-term (persisting) ice formation equivalent to a sea-level fall of almost $50 \mathrm{~m}$ (assuming $0.1 \% \circ \equiv 10$ m sea level; Labeyrie et al., 1987). Stein (1984) found a similar planktonic-to-benthic foraminifer difference in the long-term $\delta^{18} \mathrm{O}$ shift and, like Keigwin (1986), a net increase of the average planktonic $\delta^{18} \mathrm{O}$ values by about $0.5 \%$ at Site 366 near $3.0 \mathrm{Ma}$. We regard this partial glacial effect as a justification to propose the present Pliocene stage classification.

The order of magnitude of $50-\mathrm{m}$ sea-level fall compares well with observations from various Pliocene strand line and seismic sections on tectonically stable continents (e.g., Haq et al., 1987; Michel, 1973). A possible formation of a major polar ice sheet on the Northern Hemisphere on Greenland near 3.1 $\mathrm{Ma}$ (such as reported from Iceland by McDougall and Wensink, 1966, and Einarsson and Albertsson, 1988) effects a sea level drop of only $6 \mathrm{~m}$ and, therefore, must have been paralleled by a contemporary increase in polar ice volume on Antarctica, although little is known about the precise Antarctic glaciation history during the Pliocene (Harwood, 1985). The polar ice volume was largely withdrawn from the effect of short-term interglacial melting phases such as during the early Holocene, as shown by our $\delta^{18} \mathrm{O}$ curves.

\section{Carbon Isotope Record}

The dissolved oxygen and $\mathrm{CO}_{2}$ content of nonupwelled surface water is in balance with the atmosphere; hence, the water has a high content of dissolved oxygen and the dissolved $\mathrm{CO}_{2}$ has a carbon isotopic composition of about $1.0 \%$ (Duplessy, 1972; Kroopnick, 1980). In the subsurface water along the thermocline and in deep water, dissolved oxygen is utilized by the oxidation of particulate organic matter sinking from the sea surface. The addition of isotopically light organic carbon averaging about $-21 \%$ (Fontugne and Duplessy, 1981) to the deep water causes the average dissolved $\mathrm{CO}_{2}$ to become isotopically lighter.

In deep water, this process starts from a time-specific composition of the source water and gradually advances as the water passes through the deep ocean basins and "ages" (Duplessy et al., 1980; Kroopnick, 1980). Along the thermocline the advection of organic matter depends almost exclusively on the local carbon flux and the fertility of the nearby overlying surface water. In both cases, the intensity of oceanic primary productivity, which is largely controlled by upwelling in low and middle latitudes, forms a crucial factor for the $\delta^{13} \mathrm{C}$ budget of the water mass (Sarnthein et al., 1987, 1988).

The planktonic carbon isotope records of Sites 658 and 659 show high-frequency variations that largely, although not entirely, parallel the climatic cyclicity of the last $3 \mathrm{Ma}$ (Figs. $4 \mathrm{~B}$ and $5 \mathrm{~B}$ ). Warm $\delta^{18} \mathrm{O}$ stages are generally linked to positive $\delta^{13} \mathrm{C}$ events, while cold stages and the deglacial phases (except for stages 2 and 3) parallel $\delta^{13} \mathrm{C}$ minima. This relationship is more clearly depicted by the high-resolution record of Site 658 than by the record at Site 659 . The planktonic $\delta^{13} \mathrm{C}$ maxima of Site 658 reach about the same height as that of Site 659 , but the $\delta^{13} \mathrm{C}$ minima are up to $1.0 \%$ more negative, consistent with the location of Site 658 below coastal upwelling. We infer from this distribution pattern that the history of organic carbon flux, surface ocean productivity, and upwelling offshore from northwest Africa were closely linked to climatic variability.

The benthic $\delta^{13} \mathrm{C}$ record of dissolved $\mathrm{CO}_{2}$ in the bottom water at Site 658 closely parallels the fluctuations of the planktonic record of $\mathrm{CO}_{2}$ concentration near the thermocline (Fig. 4B). However, the benthic record is ${ }^{13} \mathrm{C}$-enriched by $0.5-1.0 \%$ relative to the planktonic record during joint phases of medium to strong ${ }^{13} \mathrm{C}$ depletion during the last $270,000 \mathrm{yr}$ ( $\delta^{18} \mathrm{O}$ event $8.4,38 \mathrm{mbsf}$ ). There is no systematic deviation between the two records from 270,000 to $630,000 \mathrm{yr}$ (event 16.2, 93 mbsf) ago. During the ${ }^{13} \mathrm{C}$ minima of the early Pleistocene below 99 mbsf, the benthic record is mostly ${ }^{13} \mathrm{C}$-depleted by $0.5-1.0 \%$ relative to the planktonic record. 
Moreover, many oscillations in the benthic record differ from the planktonic record. The two $\delta^{13} \mathrm{C}$ records of Site 659 (Fig. 5) show a similar distribution pattern, although they are more disturbed by single-datum fluctuations.

In summary, we observe three regimes (0-270 k.y., 270630 k.y., $1575-2000$ k.y.) of $\delta^{13} \mathrm{C}$ offset between the bottom water and subsurface water records. They suggest that the (North Atlantic) bottom water at Sites 658 and 659 received an important portion of its dissolved $\mathrm{CO}_{2}$ content via the local particulate carbon flux directly from the overlying thermocline and sea surface. This portion increased during the Brunhes Chron, especially after 270 k.y., during phases of high upwelling productivity. On the other hand, the main proportion of the dissolved bottom-water $\mathrm{CO}_{2}$ was supplied by lateral North Atlantic Deep Water advection during the early Pleistocene, when the $G$. inflata record fluctuated more independently and was relatively ${ }^{13} \mathrm{C}$-enriched, indicating a lower $\mathrm{CO}_{2}$ concentration near the thermocline.

The late Pliocene benthic carbon record of Site 658 (Fig. 3B) shows several phases of extreme ${ }^{13} \mathrm{C}$ depletion at 2.0-2.4 Ma (such as at Site 552; Shackleton et al., 1984). Minor fluctuations at a moderately ${ }^{13} \mathrm{C}$-enriched level also occurred from 2.4 to $3.3 \mathrm{Ma}$. This otherwise almost constant range was interrupted by two very short but well-documented events of extreme ${ }^{13} \mathrm{C}$ depletion at stages $128(3.046 \mathrm{Ma})$ and $137(3.32$ $\mathrm{Ma}$ ). These $\delta^{13} \mathrm{C}$ excursions are also found at Site 552 (Shackleton et al., 1984).

\section{CONCLUSIONS}

The oxygen and carbon isotope stratigraphy at Sites 658 and 659 obtained undisturbed climatic records from hemipelagic sediments of the northwest African continental margin. These records span the late Pliocene and Quaternary and are interrupted by one major and three minor stratigraphic gaps at Site 658. Upwelling-induced high productivity, combined with an ongoing hemipelagic terrigenous sediment supply, produced sedimentation rates from 8 to $>15 \mathrm{~cm} / \mathrm{k}$.y. at Site 658 . These rates provide an unprecedented time resolution of high-frequency and high-amplitude isotope oscillations that are negligibly obscured by bioturbation during the last 730,000 $\mathrm{yr}$ and from $\sim 1.6$ to 2.2 and 2.3 to $3.4 \mathrm{Ma}$.

On the basis of this study, we arrived at the following conclusions:

1. The standard sawtoothlike character of the $100-\mathrm{k} . \mathrm{y}$, climatic cycles during the Brunhes Chron is enhanced and further extended by the finding that $\delta^{18} \mathrm{O}$ event 7.5 was more negative than successive events 7.3 and 7.1 , and by the discovery of a new "aborted" ice age: the short but extremely positive oxygen isotope range of event 13.2. Based on our new evidence, event 5.1 was as warm as 5.5 .

2 . The high-resolution planktonic and benthic oxygen isotope records of Site 658 indicate numerous new high-frequency variations in climatic history at periods of 5-10 k.y. Within this frequency band, short-term climatic excursions such as the Younger-Dryas and Alleröd are shown to have accompanied most glacial terminations since approximately 630 k.y. This suggests a simple internal climatic feedback mechanism as their origin.

3. Based on the high time resolution at Site 658 and on nannofossil and magnetostratigraphic time control, we have successfully applied the new oxygen isotope stage taxonomy of Raymo et al. (1988) back to $2.75 \mathrm{Ma}$ and extended it by 21 stages back to about $3.3 \mathrm{Ma}$. The climatic variability decreased from 1.6 to $\sim 1.90 \mathrm{Ma}$. Before $1.90 \mathrm{Ma}$, several marked 100-k.y. and 40/20-k.y. climatic cycles (stages 78-114) span the time from 2.0-2.7 Ma. Hence, the first major glacial event at Site 658 predates the one found at Site 552 by about 300 k.y. Small-scale $40-$ and 100-k.y. oscillations (stages 116-137) depict less-pronounced climatic cycles between 2.7 and $3.3 \mathrm{Ma}$. A unidirectional increase in both the interglacial and glacial $\delta^{18} \mathrm{O}$ range by $0.5 \%$ near 3.0/3.15 Ma suggests an early important event of long-term polar ice formation and concomitant deep-water cooling.

4. Oxygen-isotope stratigraphy indicates that the great, slump-induced hiatus at Site 658 spans $\delta^{18} \mathrm{O}$ stages $20-59$ $(840,000 \mathrm{yr})$. A second hiatus causes the loss of events 17.2-18.3. Events $3.33-4.0$ are missed because of a stratigraphic gap in Section 108-658B-2H-6, and stages 89-95 because of a coring gap in Core 108-658A-18H.

5. The carbon isotope record of the planktonic species $G$. inflata shows that the major quasicyclic oscillations in $\delta^{13} \mathrm{C}$ and dissolved $\mathrm{CO}_{2}$ near the thermocline parallel the climatic stages of the last $2 \mathrm{Ma}$. Most likely, the $\delta^{13} \mathrm{C}$ cycles result from periodic variations in near-shore upwelling productivity near Site 658 . The benthic carbon isotope record since early stage 15 , especially during the last $270,000 \mathrm{yr}$, indicates that the ambient bottom water of Site 658 has received a major portion of its dissolved $\mathrm{CO}_{2}$ from particulate carbon originating in the overlying upwelling cell. During the early Pleistocene and late Pliocene, however, the $\mathrm{CO}_{2}$ budget of the local bottom water was dominated by variations in the advection of North Atlantic Deep Water. Several pronounced phases of 12C- (i.e., $\mathrm{CO}_{2}$-enriched bottom water) parallel the early glaciation cycles 2.0-2.4 Ma and, in particular, the unidirectional $\delta^{18} \mathrm{O}$ shift near 3.3 and $3.05 \mathrm{Ma}$.

\section{ACKNOWLEDGMENTS}

The authors would like to thank the Ocean Drilling Program for the opportunity to drill and sample Sites 658 and 659 extensively. We acknowledge with thanks many fruitful discussions on the Pliocene age control points with J. Backman (Stockholm) and A. ChepstowLusty (Cambridge), who provided their unpublished nannofossil stratigraphy for our study. Furthermore, we thank M. Raymo and W. Ruddiman for leaving us their unpublished manuscript on the detailed Site $607 \delta^{18} \mathrm{O}$ record and its stage taxonomy. L. Dupont (Göttingen), J. Mienert (Woods Hole), J. Poynter (Bristol), and P. Weinholz (Kiel) supported our study by sharing their samples.

We gratefully acknowledge the cooperation with $\mathrm{H}$. Erlenkeuser and $\mathrm{H}$. Coordt, who supervised the operation of the automatized Kiel mass spectrometer with special care, and we thank U. Glahn and J. Knaack for laboratory assistance. While on board Leg 108, W. Curry provided a detailed computerized data record of the numerous gasinduced voids in the cores of Holes $658 \mathrm{~A}$ and $658 \mathrm{~B}$. W. F. Ruddiman and unknown reviewers helped to improve our manuscript by valuable comments. This study was generously supported by the Deutsche Forschungsgemeinschaft (grant Sa 207/28).

\section{REFERENCES}

Broecker, W. S., and van Donk, J., 1970. Insolation changes, ice volumes and the $\mathrm{O}^{18}$ record in deep-sea sediments. Rev. Geophys. Space Physics, 8:169-198.

Deuser, W. G., Ross, E. H., Hemleben, C., and Spindler, M., 1981. Seasonal changes in species composition, numbers, mass, size, and isotopic compositions of planktonic foraminifera settling into the deep Sargasso Sea. Paleogeogr., Paleoclimatol., Paleoecol., 33:103-127.

Duplessy, J.-C., Moyes, J., and Pujol, C., 1980. Deep water formation in the North Atlantic Ocean during the last ice age. Nature, 286:479-482.

Einarsson, T., and Albertsson, K. J., 1988. The glacial history of Iceland during the past three million years. Philos. Trans. R. Soc. London, B, 318:637-644.

Fontugne, M. R., and Duplessy, J.-C., 1986. Variations of the monsoon regime during the upper Quaternary: evidence from carbon isotopic record of organic matter in north Indian Ocean sediment cores. Paleogeogr., Paleoclimatol., Paleoecol., 56:69-88. 
Ganssen, G., 1983. Dokumentation von küstennahem Auftrieb anhand stabiler Isotope in rezenten Foraminiferen vor NordwestAfrika. Meteor Forschungsergeb., Reihe C, 37:1-46.

Ganssen, G., and Sarnthein, M., 1983. Stable-isotope composition of foraminifers: the surface and bottom water record of coastal upwelling. In Suess, E., and Thiede, J. (Eds.), Coastal Upwelling: Its Sediment Record (Pt. A): New York (Plenum Press), 99-121.

Haq, B. U., Hardenbol, J., and Vail, P. R., 1987. Chronology of fluctuating sea levels since the Triassic. Science, 235:1156-1167.

Harwood, D. M., 1985. Late Neogene climatic fluctuations in the southern high-latitudes: implications of a warm Pliocene and deglaciated Antarctic continent. S. Afr. J. Sci., 81:239-241.

Imbrie, J., Hays, J. D., Martinson, D. G., McIntyre, A., Mix, A. C., Morley, J. J., Pisias, N. G., Prell, W. L., and Shackleton, N. J., 1984. The orbital theory of Pleistocene climate: support from a revised chronology of the marine $\delta^{18} \mathrm{O}$ record. In Berger, A., Imbrie, J., Hays, J., Kukla, G., and Saltzman, B. (Eds.), Milankovitch and Climate (Pt. 1): Hingham, MA (D. Reidel), 269-305.

Keigwin, L. D., 1986. Pliocene stable-isotope record of Deep Sea Drilling Project Site 606: sequential vents of ${ }^{18} \mathrm{O}$ enrichment beginning at $3.1 \mathrm{Ma}$. In Bouma, A. H., Coleman, J. M., Meyer, A. W., et al., Init. Repts. DSDP, 96: Washington (U.S. Govt. Printing Office), 911-920.

Kroopnick, P., 1980. Isotopic fractionations during oxygen consumption and carbonate dissolution within the North Atlantic deep water. Earth Planet. Sci. Lett., 49:485-498.

Labeyrie, L., and Berger, W. H., 1987. Abrupt climatic change: evidence and implications. NATO ASI Series: Dordrecht (D. Reidel).

Labeyrie, L. D., Duplessy, J.-C., and Blanc, P. L., 1987. Variations in mode of formation and temperature of oceanic deep water over the past 125,000 years. Nature, 327:477-483.

Mankinen, E. A., and Dalrymple, G. B., 1979. Revised geomagnetic polarity time scale for the interval 0-5 m.y. B.P. J. Geophys. Res., 84:615-626.

Michel, P., 1973. Les bassins des fleuves Sénégal et Gambie, étude géomorphologique. Mem. ORSTOM, 3:752.

McDougall, I., and Wensink, H., 1966. Paleomagnetism and geochronology of Pliocene-Pleistocene lavas in Iceland. Earth Planet. Sci. Lett., 1:232-236.

Martinson, D. G., Pisias, N. G., Hays, J. D., Imbrie, J., Moore, T. C., and Shackleton, N. J., 1987. Age dating and the orbital theory of the ice ages: development of a high-resolution 0 to 300,000-year chronostratigraphy. Quat. Res., 27:1-29.

Paterne, M., Guichard, F., Labeyrie, J., Gillot, P. Y., and Duplessy, J.-C., 1986. Tyrrhenian Sea tephrachronology of the oxygen isotope record for the past 60,000 years. Mar. Geol., 72:259-286.

Pisias, N. G., Martinson, D. G., Moore, T. C., Jr., Shackleton, N. J., Prell, W., Hays, J., and Boden, G. 1984. High resolution stratigraphic correlation of benthic oxygen isotopic records spanning the last 300,000 years. Mar. Geol., 56:119-136.

Prell, W. L., 1984. Covariance patterns of foraminiferal $\delta^{18} \mathrm{O}$ : an evaluation of Pliocene ice volume changes near 3.2 million years ago. Science, 226:692-693.

Prell, W. L., Imbrie, J., Martinson, D. G., Morley, J. J., Pisias, N. G., Shackleton, N. J., and Streeter, H. F., 1986. Graphic correlation of oxygen isotope stratigraphy: application to the late Quaternary. Paleoceanography, 1:137-162.

Raymo, M. E., Ruddiman, W. F., Backman, J., Clement, B. M., and Martinson, D. G., in press. Late Pliocene variation in northern hemisphere ice sheets and North Atlantic Deep Water circulation. Paleoceanography.
Ruddiman, W. F., Sarnthein, M., et al., 1988. Proc. ODP, Init. Repts., 108: College Station, TX (Ocean Drilling Program).

Ruddiman, W. F., Raymo, M., and McIntyre, A., 1986. Matuyama 41,000-year cycles: North Atlantic Ocean and Northern Hemisphere ice sheets. Earth Planet. Sci. Lett., 80:117-129.

Sarnthein, M., Erlenkeuser, H., von Grafenstein, R., and Schröder, C., 1984. Stable-isotope stratigraphy for the last 750,000 years: "meteor" core 13519 from the eastern equatorial Atlantic. Meteor Forschungsergeb., Reihe C, 38:9-24.

Sarnthein, M., Winn, K., and Zahn, R., 1987. Paleoproductivity of oceanic upwelling and the effect on atmospheric $\mathrm{CO}_{2}$ and climatic change during deglaciation times. In Berger, W. H., and Labeyrie, L. (Eds.), Abrupt Climatic Change. Proceedings of the NATO/ NSF A.R.W. Symposium at Biviers/Grenoble, 1985: Dordrecht (D. Reidel), 311-337.

Sarnthein, M., Winn, K., Duplessy, J.-C., and Fontugne, M. R., 1988. Global variations of surface ocean productivity in low and middle latitudes: influence on the $\mathrm{CO}_{2}$ reservoirs of the deep ocean and the atmosphere during the last 21,000 years. Paleoceanography, 3:361-399.

Shackleton, N. J., 1977. The oxygen isotope stratigraphic record of the late Pleistocene. Philos. Trans. R. Soc. London, B, 280:169-182.

Shackleton, N. J., Backman, J., Zimmermann, H., Kent, D. V., Hall, M. A., Roberts, D. G., Schnitker, D., et al., 1984. Oxygen isotope calibration of the onset of ice-rafting and history of glaciation in the North Atlantic region. Nature, 307:620-623.

Shackleton, N. J., and Hall, M. A., 1983. Stable isotope record of Hole 504 sediments: high resolution record of the Pleistocene. In Cann, J. R., Langseth, M. G., Honnorez, J., Von Herzen, R. P., White, S. M., et al., Init. Repts. DSDP, 69: Washington (U.S. Govt. Printing Office): 431-441.

1984. Oxygen and carbon isotope stratigraphy of Deep Sea Drilling Project Hole 552A: Plio-Pleistocene glacial history. In Roberts, D. G., Schnitker, D., et al., Init. Repts. DSDP, 81: Washington (U.S. Govt. Printing Office), 599-629.

Shackleton, N. J., Hall, M. A., Line, J., and Shuxi, C., 1983. Carbon isotope data in core V19-30 confirm reduced carbon dioxide concentration in the ice age atmosphere. Nature, 306:319-322.

Shackleton, N. J., and Opdyke, N. D., 1976. Oxygen-isotope and paleomagnetic stratigraphy of Pacific V28-239: late Pliocene to latest Pleistocene. Geol. Soc. Am. Mem., 145:449-464.

Stein, R., 1984. Zur neogenen Klimaentwicklung und Paläoozeanography im Nordostatlantik: Ergebnisse von DSDP Sites 141, 366, 397 und 544B. [Ph.D. diss.]. Kiel Univ., Federal Republic of Germany.

Zahn, R., 1986. Spätquartäre Entwicklung von Küstenauftrieb und Tiefenwasserzirkulation im Nordostatlantik: Rekonstruktion anhand stabiler Isotope kalkschaliger Foraminiferen [Ph.D. thesis]. Kiel Univ., Federal Republic of Germany.

Zahn, R., Winn, K., and Sarnthein, M., 1986. Benthic foraminiferal $\delta^{13} \mathrm{C}$ and accumulation rates of organic carbon (Uvigerina peregrina group and Cibicidoides wuellerstorfi). Paleoceanography, $1: 27-42$.

Zimmerman, H. B., Shackleton, N. J., Backman, J., Kent, D. V., Baldauf, J. G., Kaltenback, A. J., and Morton, A. C., 1985. History of Plio-Pleistocene climate in the northeastern Atlantic, Deep Sea Drilling Project Hole 552A. In Roberts, D. G., Schnitker, D., et al., Init. Repts. DSDP, 81: Washington (U.S. Govt. Printing Office), 861-876.

Date of initial receipt: 21 March 1988 Date of acceptance: 1 December 1988 Ms 108B-159 